Review

\title{
Bone Health in the Elderly with Type 2 Diabetes Mellitus-A Systematic Review
}

Zheer Al-Mashhadi ${ }^{1,2,{ }^{+},}{ }^{*}$, Rikke Viggers ${ }^{3,4,+}$, Rasmus Fuglsang-Nielsen ${ }^{1,5}$, Bente Langdahl ${ }^{2,6}$, Peter Vestergaard ${ }^{3,4,7}$, Søren Gregersen ${ }^{1,2,6}$, Jakob Starup-Linde ${ }^{1,6}$

1. Steno Diabetes Center Aarhus, Aarhus University Hospital, Aarhus, Denmark; E-Mails: zheer@clin.au.dk; rasmunie@rm.dk; soeren.gregersen@aarhus.rm.dk; Jakob.Linde@auh.rm.dk

2. Department of Clinical Medicine, Aarhus University, Aarhus, Denmark; E-Mail: bente.langdahl@aarhus.rm.dk

3. Steno Diabetes Center North Jutland, Aalborg University Hospital, Aalborg, Denmark; E-Mails: rikke.viggers@rn.dk; p.vestergaard@rn.dk

4. Department of Clinical Medicine, Aalborg University, Aalborg, Denmark

5. Department of Internal Medicine, Regional Hospital Randers, Randers, Denmark

6. Department of Endocrinology and Internal Medicine, Aarhus University Hospital, Aarhus, Denmark

7. Department of Endocrinology and Internal Medicine, Aalborg University Hospital, Aalborg, Denmark

+ These authors contributed equally to this work.

* Correspondence: Zheer Al-Mashhadi; E-Mail: zheer@clin.au.dk

Academic Editor: P. Hemachandra Reddy

Special Issue: Diabetes in the Elderly

OBM Geriatrics

2020, volume 4, issue 2

doi:10.21926/obm.geriatr.2002123
Received: April 02, 2020

Accepted: May 29, 2020

Published: June 18, 2020

\section{Abstract}

Type 2 diabetes mellitus (T2DM) and osteoporosis are two major public health concerns worldwide, contributing to morbidity and mortality in the elderly. Aging is one of the most significant risk factors for low bone mass, bone fragility, and fractures. Among the several

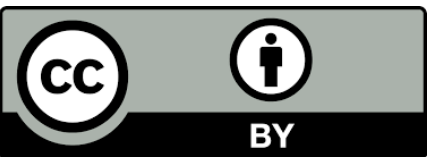

(C) 2020 by the author. This is an open access article distributed under the conditions of the Creative Commons by Attribution License, which permits unrestricted use, distribution, and reproduction in any medium or format, provided the original work is correctly cited. 
comorbidities that affect the elderly with diabetes, increased fracture risk is a relatively recently discovered complication. Generally, individuals with T2DM exhibit higher bone mineral density, which complicates the assessment of fracture risk. Despite the growing evidence for an association between T2DM and increased fracture risk, especially among the elderly, the underlying mechanism has not yet been fully uncovered, and proper evaluation of bone health in individuals with T2DM remains a challenge. The present review includes 125 articles investigating the effects of T2DM on bone health in the elderly. A systematic literature search was performed in PubMed, Embase, and CINAHL for articles containing terms corresponding to 'elderly' and 'type 2 diabetes mellitus' along with 'bone fracture', 'osteoporosis', or 'bone turnover'. Articles investigating the effects of T2DM and disease severity, duration, or complications on bone parameters - i.e. fracture risk, structure, and turnover - were selected for inclusion in the present review. Overall, the evidence indicated reduced bone turnover in individuals with T2DM, accompanied by an increased bone mineral density (BMD) and an inefficient distribution of bone mass with accumulated trabecular bone and diminished cortical bone. These structural alterations in bone tissues result in bone fragility and overall increased fracture risk in elderly individuals with T2DM. However, measurement of BMD does not adequately predict the increased fracture risk in T2DM. Therefore, identification and application of more precise predictors of fracture risk in individuals with T2DM are required. Furthermore, a better understanding of the pathophysiological mechanisms involved may assist in developing effective treatments of bone disease in individuals with diabetes. The present review introduces current candidates for improved measures of bone quality and fracture risk along with the current knowledge on the pathophysiology of diabetic bones.

\section{Keywords}

Elderly; bone; diabetes mellitus; bone structure; bone turnover; fractures

\section{Introduction}

The average human lifespan has increased by 5.5 years over the last 16 years [1]. Currently, the average life expectancy in Europe and the United States of America is 80.9 years and 78.7 years, respectively, with the highest life expectancy reported in women [2,3]. Consequently, the need for effective prevention and treatment of chronic aging-related diseases has increased. Type 2 diabetes mellitus (type 2 DM; T2DM) and osteoporosis are two common metabolic diseases among the elderly $[4,5]$.

Increasing age is a significant risk factor for osteoporosis, as evidenced by the exponential increase in hip fracture incidence with age [6]. The World Health Organization has defined the following diagnostic criterion for osteoporosis: bone mineral density (BMD) of $\geq 2.5$ SD below the average value for young, healthy women, i.e., a BMD T-score $\leq-2.5[6,7]$. The T-score is derived from areal BMD (aBMD) measured using Dual-energy X-ray Absorptiometry (DXA) at the lumbar spine, femoral neck, or total hip. However, in a prospective study from 2004 conducted with elderly men and women aged $\geq 55$ years, it was reported that only $21 \%$ and $44 \%$ of all non- 
vertebral fractures in men and women, respectively, occurred with an aBMD T-score below -2.5 [8]. Therefore, there must be several important risk factors for fractures other than a relative reduction in the aBMD.

T2DM is a chronic metabolic disorder that arises due to insulin resistance and a relative insulin deficiency, resulting in elevated blood glucose levels [9]. The risk of T2DM is reported to increase with age [10]. Neither the Fracture Risk Assessment Tool (FRAX) nor aBMD sufficiently predict fracture risk in T2DM [11, 12]. In fact, fracture risk is underestimated in T2DM, corroborating the hypothesis that bone quality is affected by T2DM $[12,13]$. T2DM is associated with an increased fracture risk despite consistent findings of unaffected or elevated aBMD [13-16]. Diabetic bones are characterized by a state of low bone turnover, which potentially leads to impaired bone quality $[17,18]$. Evidence suggests that T2DM is an independent risk factor for bone fragility and fractures as it exerts an effect on bone metabolism and aggravates the age-related impairment of bone quality [17]. Therefore, bone health in the elderly with T2DM requires increased attention and improved assessment. However, the utility of aBMD as a clinical indicator of osteoporosis and fracture risk has significant limitations. Complementary tools are increasingly being used to assess the association of diabetes to bone fragility and fractures in the elderly. In addition, a higher aBMD T-score intervention threshold has been proposed for diabetic patients [19].

The present systematic review summarizes the current knowledge regarding bone health in the elderly with T2DM through discussion of structural and biochemical bone measurements as well as fracture risk.

The aim of the present review is to present the current knowledge on the effects of T2DM on bone, identify associated risk factors and confounders, and identify knowledge gaps in the field.

\section{Materials and Methods}

A systematic literature search was performed in accordance with the PRISMA guidelines [20, 21] in the following three databases: PubMed, Embase, and CINAHL. The search strings were optimized through database-specific evaluation of the search terms. Search terms were used only if they influenced the number of articles yielded by the search. Each database has its own form of bibliographic indexing (for example, MeSH-terms in PubMed), and these indexing terms were identified for each database and added to the respective search strings. The search strings were made to yield articles satisfying the following three criteria: (1) contained a term representing "type 2 diabetes" in the title or abstract, (2) contained a term representing "elderly" in the title or abstract (or in the 'age group' category for CinAHL), and (3) contained a term representing "bone disease", "fracture", or " bone turnover" in the title or abstract. The final literature search was performed on $29^{\text {th }}$ October, 2019.

Table S1 lists the search terms used in the PubMed search. Table S2 provides the exact search strings. The initial search yielded 1,548 results. After removing duplicate articles, 1,204 articles remained. Two reviewers (RV and ZAM) independently assessed abstracts and subsequently fulltext articles for eligibility according to the inclusion criteria. Disagreements were resolved through discussion.

Figure 1 illustrates the exclusion process described ahead. First, articles which were not available in English, German, Spanish, Italian, French, Norwegian, Danish, or Swedish were excluded. Next, articles reporting studies that were not human in-vivo studies were excluded. 
Subsequently, case reports, case series, posters, conference abstracts, reviews (without metaanalysis), letters to the editor, commentaries, expert opinions, and consensus statements were excluded. Finally, the remaining abstracts and articles were assessed to identify relevant study populations, exposures, and outcomes. Articles were included only if at least one subset of the study population had T2DM, satisfied the age criterion (see below), and was examined for relevant bone-related outcomes (see below). The age criterion was as follows: mean age $\geq 55$ years, minimum age $\geq 50$ years, or postmenopausal. Exposures were considered relevant if the study involved one of the following: 1) comparison of T2DM with non-diabetic subjects, 2) analysis of the effects of glycemic control (e.g., hemoglobin A1c [HbA1c] or fasting plasma glucose [FPG]), 3) assessment of the effects of insulin resistance (e.g., through homeostatic model assessment [HOMA]), 4) investigation of the effects of prevalent diabetic complications (e.g., neuropathy), or 5) assessment of the effects of diabetes duration. Outcomes were considered relevant if the study involved: 1 ) any measure of fracture risk, 2) any measure of bone structural integrity (such as BMD, trabecular bone score [TBS], and bone material strength index [BMSi]), or 3) bone biochemical markers. Bone markers included osteocalcin (OC), sclerostin, carboxy-terminal collagen crosslinks (CTX), N-terminal propeptide of type 1 procollagen (P1NP), and osteoprotegerin, among others. Measurements of parathyroid hormone (PTH), vitamin D, and alkaline phosphatase (ALP) levels in articles were noted, although articles were not considered for inclusion if no other bone markers were measured. Finally, inaccessible full-text articles were excluded, as the categories of data considered relevant for extraction could not be reliably obtained from abstracts. Conference abstracts were excluded for the same reason. Articles were regarded as inaccessible if there were no links to the articles in the utilized databases; authors were not contacted. Eleven additional articles eligible for inclusion were identified in the reference lists of the examined articles and were included in the final review.

Two authors, RV and ZAM, extracted data from all the included articles; articles involving bone structural parameters were examined by author RV, while articles involving biochemical or fracture-related parameters were examined by author ZAM.

Data related to the following were extracted: 1) author name, 2) study sample size, 3) population characteristics (age, nationality/geographic information, sex, disease status, other relevant information), 4) investigation performed or main outcome parameter (for example, DXAscan/aBMD), 5) adjustment parameters used in the main statistical analyses, and 6) major findings, including significance levels and effect sizes.

The extracted data for biochemical, structural, and fracture-related outcomes are presented in Table 1, Table 2, and Table 3, respectively. If solely the baseline characteristics from a study population were extracted for review, the study in question was characterized as cross-sectional, regardless of the true nature of the study. 


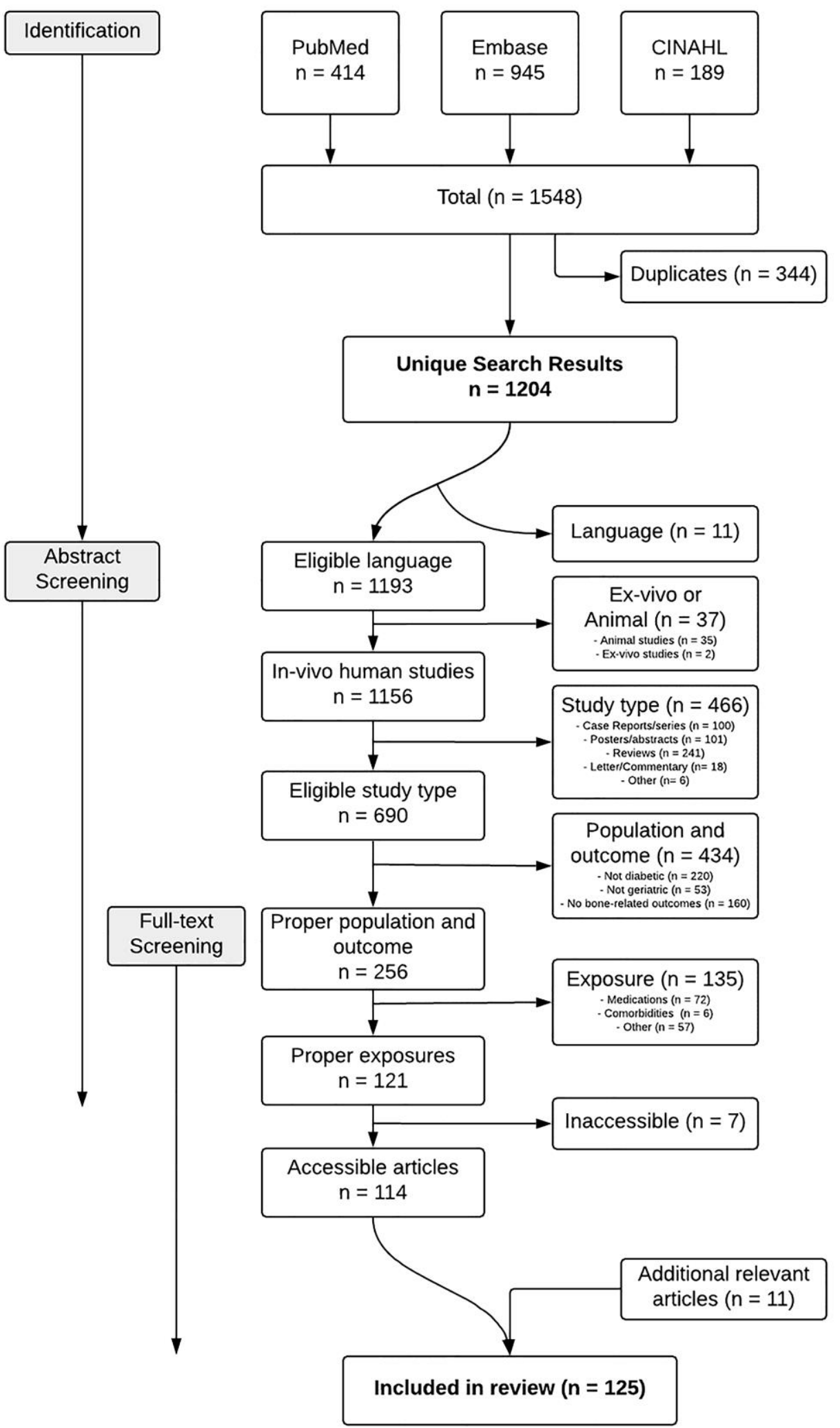

Figure 1 Appraisal/exclusion process. 


\section{Results}

A total of 125 articles satisfied the inclusion criteria and were included in the present review. Among the included articles, 78 articles investigated bone structural changes associated with T2DM, 61 articles investigated fracture risk, and 28 articles investigated bone biochemical markers.

\subsection{T2DM and Biochemical Markers of Bone Health}

Among the 28 articles investigating the relationship between T2DM or related parameters and bone biochemical markers (Table 1), 7 articles included only men and 11 included only women as their study subjects. In the remaining 10 articles, the average proportion of women was 55\% (not weighted according to study size). In the studies reporting age, the mean ages were averaged (not weighted according to study size) to 58 years.

Only two studies reported no association between T2DM and related parameters (duration, complications, or severity) and bone biochemical markers [22, 23]. The most commonly studied markers were OC, sclerostin, CTX, and P1NP. OC and P1NP are markers of osteoblast function and bone formation, while CTX and NTX (N-terminal telopeptide) are collagen degradation products and, therefore, bone resorption markers [24]. Levels of undercarboxylated OC (uCOC) are associated with increased fracture risk [25]. Sclerostin is an inhibitor of osteoblasts and, consequently, bone formation [24].

Several studies reported lower levels of CTX or NTX in individuals with T2DM [26-34], those with diabetes-related retinal complications [35], or those with increasingly pathological levels of glycemic markers $[31,36]$. Other studies reported no association between these markers and diabetes [23, 36-40]. Zhou et al. reported an increased urinary NTX secretion in individuals with T2DM [41], whereas Ardawi et al. observed reduced urinary NTX secretion in these individuals [34]

Several studies reported suppressed OC levels in individuals with T2DM [26, 27, 29-32, 34, 37$39,41-45]$. Suppressed OC levels have also been reported in association with higher levels of FPG, fasting plasma insulin (FPI), HbA1c, or HOMA-IR (HOMA-insulin resistance) or with lower levels of HOMA- $\beta$ (HOMA - beta-cell function) [26, 31, 42-44, 46, 47]. Xia et al. compared different groups of individuals with T2DM and reported decreased OC levels in the group with microalbuminuria; the OC levels were observed to be further decreased in the group with macroalbuminuria [48].

Yeap et al. reported that the ratio of ucOC to total OC was higher in individuals with T2DM compared to those without DM [29]. Iki et al. observed that this ratio increased with higher levels of FPG, FPI, HOMA-IR, and HbA1C [44]. Few studies reported no association between $\mathrm{OC}$ and T2DM $[22,23,33]$. Bulló et al. reported positive associations of baseline OC levels with FPI, HOMA$I R$, and HOMA- $\beta$, although changes in OC levels over a period of two years (during a dietintervention randomized controlled trial) could not predict FPG or FPI levels [47].

Similarly, P1NP was observed to be reduced in individuals with T2DM [26-30, 34, 37, 39, 49], those with diabetes-related retinal complications [35], and those with higher levels of FPG, FPI, HbA1c, or HOMA-IR or lower levels of HOMA- $\beta[36,49]$. Few studies reported no effects of T2DM on P1NP levels [36, 38].

Among the six studies investigating sclerostin, two studies reported increased levels of sclerostin in individuals with $\operatorname{T2DM}[33,34]$. In addition, García-Martín et al. reported an association between sclerostin levels and the duration of T2DM [33]. Three studies reported no 
correlation between T2DM or $\mathrm{HbA} 1 \mathrm{c}$ and sclerostin levels [22, 38, 46]. Only one study reported lower levels of sclerostin in individuals with T2DM or in those with higher levels of FPG or FPI [42].

Rianon et al. studied the effect of DM duration and reported no association with OC or sclerostin levels [46]. Rasul et al. reported an association between prevalent polyneuropathy and higher levels of CTX, P1NP, and OC in men but not in women [50]. Maagensen et al. and Chailurkit et al. investigated the effect of an oral glucose load and reported that the suppression of CTX was attenuated in individuals with T2DM [37, 40]. Moreover, Maagensen et al. observed that the suppression of OC and P1NP was unaffected in individuals with T2DM, and Chailurkit et al. observed the suppression of osteoprotegerin to be unaffected by T2DM [37, 40].

Most of the studies investigating levels of vitamin D, PTH, calcitonin, or ALP reported no significant differences between individuals with DM and those without DM [23, 26, 28, 31-33, 35, $36,41,48,50,51]$. However, decreased vitamin D levels in individuals with T2DM were reported in three studies [32, 36, 38], whereas increased levels were reported in one [30]. Four studies observed no effect of DM on vitamin D levels $[26,28,31,51]$. Three studies reported lower PTH levels in individuals with T2DM [30, 31, 34, 39], whereas in this population PTH levels were reported to be higher in one study [48] and unaffected in seven studies [23, 26, 28, 32, 36, 41, 51] compared to individuals without DM.

\subsection{T2DM and Structural Changes in Bone Tissue}

Among the 78 articles that reported outcomes related to bone structure (Table 2), 12 included only men and 30 included only women as their study subjects. In the remaining 36 articles, the average proportion of women was $58 \%$ (not weighted according to study size). In the studies reporting age, the mean ages were averaged to 65 years (not weighted according to study size).

There was considerable variation among the articles in regard to the measures of bone structure used, with a variety of measures for BMD, bone composition, and bone microarchitecture.

The most prevalent among the methods and outcome parameters in the examined articles was DXA/aBMD ( $\mathrm{N}=64)$, followed by Quantitative Computed Tomography (QCT) or High-Resolution peripheral QCT (HRpQCT) $(N=9)$, Quantitative UltraSound (QUS) $(N=10)$, and $M R I(N=3)$, respectively.

Among the 78 studies involving structural bone measurements, 66 studies reported the effects of T2DM or glycemic control on aBMD, primarily obtained by DXA-scan of total hip, femoral neck, or lumbar spine. In 50 studies, higher aBMD at one or more sites was observed in association with prevalent T2DM or with worse glycemic control in individuals with T2DM. Higher aBMD was observed at all measured sites in 16 studies. Nine studies reported no difference in aBMD, while five studies reported lower aBMD in individuals with T2DM compared to those without T2DM (details below).

A few studies reported no difference in aBMD between individuals with and without T2DM [26, 33, 52-58]. Although these study populations were dissimilar in terms of nationalities and sex, all of these studies were adjusted for multiple variables-always including BMI. On the other hand, the studies reporting a difference were more heterogeneous in terms of statistical analyses, and a number of results were based on unadjusted data, although the majority of the studies were adjusted for BMI and other factors (Table 2). 
Five studies, four of which included Chinese men and women as study subjects, reported reduced aBMD in correlation with T2DM or higher HbA1c levels $[41,48,59,60]$. Sert et al., in their study conducted on Turkish men and women, reported that T2DM was associated with reduced $\mathrm{aBMD}$ at the lumbar spine in men, whereas higher femoral aBMD was reported in both men and women [61]. The mean BMI values were in the range of 25.1-26.1 in three studies with Chinese subjects $[48,59,60]$, and Zhou et al. reported reduced aBMD only in those subjects which had BMI $<25 \mathrm{~kg} / \mathrm{m}^{2}$ [41]. These findings may indicate that T2DM is associated with increased BMD only in the presence of high BMI. However, six other studies conducted on Asian populations did report increased aBMD in subjects with T2DM with mean BMI ranging from 22.0 to 25.6 [30, 39, 62-65].

A higher trabecular bone score (TBS) reflects denser trabeculae and a more fracture-resistant microarchitecture [66]. TBS is estimated from DXA and has been reported to be associated with osteoporotic fracture risk, independently of aBMD and clinical risk factors $[67,68]$. Studies have reported lower TBS in subjects with T2DM compared to those with prediabetes and those with normal glucose metabolism [39, 58, 69, 70]. Especially lumbar spine TBS appears to be reduced in $\operatorname{T2DM}[58,69,71]$. This is consistent with the findings of a meta-analysis conducted by Ho-Pham et al., which concluded that individuals with T2DM exhibit lower TBS compared to those without DM [72].

Leslie et al. reported an accentuated risk of incident major osteoporotic fracture after adjusting for BMD, whereas the risk was partially attenuated when adjusting for trabecular bone score (TBS) [71]. This supports the conclusion that BMD is insufficient as a prognostic tool for predicting fracture rates in individuals with T2DM. TBS may thus serve as an easily obtainable yet powerful addition to any predictive model for fracture risk in T2DM.

It is not possible to distinguish between trabecular and cortical BMD using DXA. Cortical bone density is a major determinant of bone strength. QCT and HRpQCT enable the assessment of bone architecture at the trabecular and cortical sites using volumetric BMD (VBMD) and the prediction of bone fragility and fracture risk $[73,74]$. VBMD was measured in nine of the included studies.

Several studies reported VBMD differences, such as higher spine VBMD [75] and radial and tibial trabecular VBMD $[28,76,77]$ in subjects with T2DM compared to those without DM. A few studies reported higher $\mathrm{ABMD}$ in T2DM without observing any difference in $\mathrm{VBMD}$ [78-80]. One study reported lower VBMD at the spine in individuals with T2DM compared to those without DM [81]. De Waard et al. observed no association between HRpQCT measures of VBMD and T2DM or prediabetes, although they reported an association between $\mathrm{HbA1c}>7 \%(53 \mathrm{mmol} / \mathrm{mol})$ and higher cortical porosity at the distal radius in individuals with T2DM [79].

Several studies reported higher cortical porosity [77, 79] and lower cortical thickness [79, 82] and $V B M D[76,79]$ in individuals with T2DM. Nakamura et al. observed higher trabecular vBMD (measured by QUS) in individuals with T2DM after the age of 60 compared to individuals without DM [82]. A few studies reported no difference in cortical thickness (Table 2), while one study reported lower cortical porosity in individuals with T2DM [36].

A study that employed MRI detected higher bone marrow fat content in T2DM, which correlated inversely to hip aBMD [78]. Another study employing MRI reported increased spacing within the trabecular bone network in subjects with T2DM [83]. A prospective cohort study with two years of follow-up did not report any differences in the evolution of trabecular bone microarchitecture in elderly women with T2DM compared to elderly women without DM [84]. 
All the included studies were observational apart from one study, which was a randomized controlled trial [52]. The latter investigated whether bone health could benefit from intensive glycemic control, but no difference in $\mathrm{ABMD}$ was observed at any site following intensive or standard glycemic control with a median intervention time at examination of 2.2 years [52].

In contrast to these findings in individuals with T2DM, a study conducted by Napoli et al. in 2019 examined aBMD in individuals without DM and observed no association with insulin sensitivity assessed through HOMA-IR [85]. This may indicate a dose- and time-dependent effect of insulin resistance on bone quality, which becomes apparent only after the development of T2DM.

In summary, the studies included in the present review were substantially heterogeneous in terms of bone structural outcome measures, sex-distribution, and demographics. Increased aBMD was reported in both men and women with T2DM. Most of the included studies reported a positive association of aBMD with higher glucose or $\mathrm{HbA1c}$ levels, whereas a few studies reported a negative association with DM duration even after adjusting for age. However, it is not possible to distinguish between trabecular and cortical bone architecture using $\mathrm{aBMD}$, which is only one component of bone quality. The VBMD measurements in the included studies indicated a greater trabecular bone volume and increased cortical porosity in elderly persons with T2DM. However, there is no clear consensus on which bone site is the most affected by diabetes-related structural changes. Therefore, the elderly with DM may have impaired bone quality which cannot be assessed properly using routine diagnostic methods, i.e. DXA BMD and T-score thresholds.

\subsection{Changes in Bone Structure over Time in T2DM}

Although there is an apparent association between prevalent T2DM and higher aBMD, a study conducted by Jang et al. reported that subjects with diabetes duration > five years had approximately the same adjusted femoral neck aBMD $\left(0.738 \pm 0.004 \mathrm{~g} / \mathrm{cm}^{2}\right)$ as subjects without T2DM, which was in turn lower than that of subjects with shorter diabetes duration $(0.773 \pm 0.004$ $\mathrm{g} / \mathrm{cm}^{2}$ ) after adjusting for age, body mass index (BMI), and other factors [86]. Leslie et al. observed that BMD loss was $0.0018 \mathrm{~g} / \mathrm{cm}^{2} /$ year greater in individuals with T2DM compared to normalweight individuals without DM (age-adjusted) [87]. A study conducted by $\mathrm{Xu}$ et al. reported a crude odds ratio (OR) of 2.06 for osteoporosis with diabetes duration $>20$ years compared to duration < 10 years, although this effect was fully attenuated after adjusting for age, BMI, and a variety of other factors [88]. In addition, Xu et al. reported an adjusted OR of 1.63 for osteoporosis in association with poor glycemic control ( $\mathrm{HbA1c} \geq 7.5 \%)$ in males but not in females [88].

In contrast, Shan et al. reported a smaller aBMD decrease at the lumbar spine with increasing age in individuals with T2DM compared to those without DM; in addition, a less pronounced increase in osteoporosis prevalence was observed with increasing age [64].

Taken together, these findings appear to suggest an increase in the rate of bone loss with age. However, it is difficult to reconcile this finding with reduced bone turnover and increased BMD. Rather, it may be that an increased mineral loss is present only in a subset of individuals with T2DM, possibly in those with the highest severity and longest duration of the disease, allowing for several years of mineral accumulation prior to the acceleration of mineral loss at a late stage. 


\subsection{T2DM and Risk of Fracture}

Among the 61 articles investigating the effects of T2DM on fracture risk (Table 3), 6 included only men, and 16 included only women as their study subjects. In the remaining 39 articles, the average proportion of women was $55 \%$ (not weighted according to study size). In the studies reporting age, the mean ages were averaged to 70 years (not weighted according to study size).

Most articles $(\mathrm{N}=49$ ) reported an increased risk of fracture in at least one location associated with the presence of diabetes or diabetes-related parameters. A total of 13 studies reported no association of fracture risk with $\operatorname{T2DM}[32,75,89-92], \mathrm{HbA1c}$ levels [38, 62, 80, 93-96], fasting plasma glucose [38,62], HOMA indices [90], or diabetes duration [32, 62, 80, 95]. Five of these studies reported no associations in any of the analyses performed [32, 38, 75, 89, 93]. Six studies reported reduced fracture risk in at least one location associated with the presence of diabetes [51, 81, 95, 97-99] or higher HbA1c levels [97]; four of these studies were relatively small (the largest with $n=5,931$ ) case-control studies investigating fracture prevalence [51, 81, 95, 99], whereas the remaining two were retrospective cohort studies investigating fracture incidence [97, 98].

All the studies included in the present review were observational apart from one randomized controlled trial which reported no effect of intensive glycemic control on fracture risk compared to standard glycemic control [52].

Several studies reported an increase in hip fracture prevalence or incidence with the presence of T2DM ( $N=22)[14,31,65,100-118]$, higher levels of HbA1c in individuals with T2DM (cut-offs varying among the studies) $(N=6)[91,107,119-122]$, higher fasting plasma glucose in individuals with T2DM ( $N=1)$ [120], complications of diabetes $(N=5)[94,102,107,109,121]$, or duration of diabetes $(N=8)[14,107,110,114,115,118,120,121]$. de Liefde et al. reported no association between T2DM and hip fracture, although they did observe an increased risk of hip fracture associated with diabetes treated with antidiabetic drugs [123]. Among the studies which reported association of T2DM with an increased risk of hip fracture, most presented an OR, a hazard ratio $(H R)$, a relative risk (RR), or an incidence rate ratio (IRR). Most of the effect sizes reported here (OR/HR/RR/IRR) were in the range of 1.30-1.80. The largest studies were conducted by Hothersall et al. ( $n=3,840,841$ ) and Hippisley-Cox et al. ( $n=3,142,673$ ), which in women reported HRs of 1.05 (95\% confidence interval $[\mathrm{Cl}]$ of $1.01-1.10)$ and 1.57 (95\% Cl 1.45-1.69), respectively [110, 112]. A meta-analysis of 12 articles (combined $n=764,282$ ), which included several of the studies examined in the present review [31, 32, 89, 113-115, 118, 123], conducted by Dytfeld et al. assessed hip fracture risk and revealed an increased risk with an OR of $1.30(95 \% \mathrm{Cl} 1.07-1.57)$ [104]. Only one study reported a reduced risk of hip fracture associated with T2DM; however, this association was observed only in the subgroup comprising males not receiving antidiabetic medications [124].

Vertebral fracture risk was reported to be associated with diabetes in six studies $[30,34,62,96$, $105,125]$, among which five studies investigated prevalent fractures using x-ray imaging [30, 34, $62,96,125]$. Majumdar et al. studied incident fractures by means of hospital discharge notes and reported an increased risk with a study sample of $n=57,938$ [105]. Yamamoto et al. reported a higher prevalence of vertebral fracture (evaluated using $x$-ray) in women with T2DM, while a lower prevalence was reported in men with T2DM (although no statistical analysis was performed) [30]. Viégas et al. reported an increased prevalence of vertebral fracture associated with longer 
diabetes duration and with the presence of retinopathy; the increase was not observed for nephropathy and peripheral diabetic neuropathy [125]. Only two of the studies reported ORs [62, 96]. Kilpadi et al. reported an OR of 2.86 (95\% Cl 1.56-5.34), and Yamamoto et al. reported an OR of 1.86 (95\% Cl 1.11-3.12) for women and 4.73 (95\% Cl 2.19-10.20) for men; however, both of these studies had a relatively small sample size ( $n=296$ and $n=996$, respectively) $[62,96]$. In contrast, eight studies reported no association of vertebral fracture risk with diabetes $[14,32,75$, $89,103,111]$ or glycemic control [38, 62]. Among these eight studies, six studies investigated prevalent fractures $[32,38,62,75,89,111]$ and four studies investigated incident fractures $[14,32$, $75,103]$, most by means of x-ray assessment, although two studies relied on self-reported data $[38,111]$. A meta-analysis by Dytfeld et al. of seven studies (combined $n=107,514$ ), which included four of the above-stated studies [14, 32, 62, 89], investigated vertebral fracture risk and did not observe this to be increased (OR $=1.13$ [95\% Cl 0.94-1.37]) [104]. No studies reported a reduced risk specifically of vertebral fracture in individuals with T2DM.

Many articles investigated the overall risk of fracture or risk of clusters of fracture types, such as non-vertebral fractures, osteoporotic fractures (OPF), major osteoporotic fractures (MOPF), and non-hip non-vertebral fractures (NHNVF). The majority of these articles reported an increased risk associated with diabetes [14, 69, 71, 80, 102, 105, 108, 111-113, 123, 126-130], with glycemia in individuals with T2DM (higher levels of HbA1c or FPG, with cut-offs varying among studies) [69, 90, 128], with longer disease duration [92, 105, 123, 131], or with diabetic complications [92, 102]. Few articles reported no increased risk of overall or clustered fracture types $[38,51,81,89,93,95$, $97,99,103]$. In the articles that reported an effect on the overall risk of clustered fracture types, effect sizes (ORs or HRs) were around 1.20 [102, 108, 112]. Kachroo et al. observed that each 1 standard deviation (SD) increase in HbA1c or FPG levels was associated with an HR of 1.38-1.39 for osteoporotic fractures and an HR of 1.45-1.48 for major osteoporotic fractures [90].

Reporting of fracture risk in locations other than the hip or vertebrae was less common (data presented in Table 3).

In summary, there appears to be a general trend toward an increased risk of hip and overall fractures in elderly individuals with T2DM compared to individuals without T2DM. This finding is consistent with the conclusion of a literature review conducted by Rasmussen et al. [132]. The severity of DM, measured in terms of glycemic control, diabetes duration, and complications, also appears to be a risk factor for fractures. The evidence for increased risk of vertebral fractures is inconsistent, although all the studies included in the present review reported either no effect or an increased risk of fracture in this location. However, the observed increase in fracture risk may also be mediated, at least in part, by an increased risk of falls due to hypoglycemia and peripheral neuropathy.

\subsection{Bone Health and Hypoglycemia}

Although hyperglycemia appears to be a risk factor for fractures, some studies reported an increased risk of fractures associated with hypoglycemia or with lower levels of $\mathrm{HbA1c}$ compared to higher HbA1c levels [119, 125, 133-135], whereas only two studies reported no deleterious effect of hypoglycemia [120,121]. However, Chiang et al. observed that visit-to-visit variation of FPG was a predictor of hip fracture, indicating an effect mediated by increased hypoglycemia risk [120]. Kachroo et al. quantified the increased risk associated with hypoglycemia and reported an 
OR of 2.16 (95\% Cl 1.74-2.67) for any fracture [134]. Viégas et al. observed that normal postprandial glucose in individuals with T2DM was a risk factor for vertebral fractures compared to postprandial hyperglycemia and that women with prevalent fractures had lower levels of postprandial glucose and HbA1c compared to those without prevalent fractures [125]. This may reflect an underlying increased risk of hypoglycemia in those with normal postprandial glucose levels in this particular study population. Two studies reported that the increased fracture risk was entirely or mostly associated with insulin use, suggesting an effect mediated in part by hypoglycemia [124, 129]. Another study reported that the increased fracture risk was fully attenuated after adjusting for comorbidities and falls [127]. It is a seemingly inconsistent finding that fracture groups exhibit higher HbA1c levels in some studies and lower HbA1c levels in other studies. However, this apparent contradiction may be explained by a J-shaped association mediated by hypoglycemia and falls at one end and bone fragility at the other. Indeed, Lee et al. reported just such an association with increased fracture risk at both high (>9.5\%) and low $(<6.5 \%)$ HbA1c levels [119].

\subsection{Bone Health and Complications of T2DM}

A few studies reported data on diabetes-related complications and bone structure. Xia et al. reported that a lower $\mathrm{aBMD}$ at the lumbar spine and femoral neck was correlated with albuminuria in individuals with T2DM [48]. Rasul et al. observed no differences in aBMD between individuals having T2DM with and without polyneuropathy [50]. Zhong et al. reported no association between $\mathrm{aBMD}$ and $\mathrm{HbA1c}$ levels, although it was observed that the presence of microangiopathy was associated with decreased bone mass at the lumbar spine, total hip, and femoral neck in women, while the same was not true for men [136].

A total of seven studies reported an increased risk of fractures associated with the presence of diabetes-related complications [92, 94, 102, 107, 109, 121, 125]. An increased risk of fractures associated with the presence of peripheral neuropathy was reported in three studies, the largest of which was conducted by Lee et al. ( $n=2,798,309)[102,107,121]$. Three smaller studies reported no effect of peripheral neuropathy on fracture risk, possibly due to insufficient sample size $[62,120,125]$. In addition, these three small studies reported no effects of nephropathy [125] or retinopathy $[62,120]$, whereas four studies (the largest conducted by Kabue et al.; $n=120,256$ ) observed that the presence of retinopathy was associated with fracture risk [92, 94, 121, 125]. Reyes et al. (registry-based study) observed that T2DM with any complications (of unspecified type) presented an increase of $89 \%$ in hip fracture risk compared to T2DM without complications, which presented an increase of only $45 \%$ in hip fracture risk.

These findings suggest that all complications of diabetes, besides serving as simple proxies for disease severity, may increase fracture risk independently. However, retinopathy and peripheral neuropathy may have a special significance in increasing the risk of fractures, as the impaired vision and proprioception is expected to increase the risk of falling.

\subsection{Sex-Specific Differences in Bone Health in T2DM}

It is well known that men have a lower risk of osteoporosis and fractures and a higher BMD compared to women [7]. Nonetheless, sex-specific differences regarding bone mass in individuals 
with T2DM have been investigated inadequately. The vast majority of studies reported increased aBMD associated with T2DM in both men and women (Table 2).

However, a number of studies included in the present review concluded that women with T2DM had a higher aBMD compared to women without DM, whereas no such difference was observed for men [30,88, 137-139]. A cohort study conducted by Lunt et al. investigated the effect of insulin use in T2DM and reported that insulin treatment was associated with an increase in $\mathrm{aBMD}$ at all sites in women, whereas such an increase was observed only at the spine in men [140].

Only one study reported higher whole-body aBMD in men with T2DM and not in women with T2DM [141]. Chi et al. reported a higher aBMD in men compared to women, independently of the presence of diabetes [142]. Sert et al. observed that the difference in aBMD between subjects with T2DM and those without DM was more pronounced in men than in women [61]. The study reported that among women aged 51-60, those with T2DM exhibited higher femoral neck aBMD than controls [61]. Similarly, among men aged 51-60, those with T2DM exhibited higher femoral neck and total femur aBMD and lower lumbar spine aBMD compared to men without DM, the latter being present already after the age of 30 [61].

A study comparing men and women with and without T2DM reported that T2DM was associated with skeletal hypertrophy (measured using HRpQCT), which was attenuated at the tibial cortex in men compared to women [143].

Accounting for sex altered the strength of the association between T2DM and fracture risk in some studies $[65,98,111,113]$, although the direction of this effect was not consistent across studies. Wallander et al. reported that the risks of any fracture, hip fracture, and major osteoporotic fractures were all increased to a higher extent in women compared to men [124].

\subsection{Effects of Other Subject Characteristics}

Several articles examined the effects of other participant characteristics on the association between T2DM and fracture risk.

Only two studies investigated the effect of T2DM on fracture risk in individuals with osteoporosis [116, 126]. Sato et al. reported an association between T2DM and fracture risk in individuals diagnosed with osteoporosis and receiving antiosteoporotic medication [126], whereas Taylor et al. reported no association between T2DM and fracture risk in the subset of osteoporotic participants [116].

Two studies examining bone structure reported that aBMD was increased only in the obese subpopulation [137] and that obesity attenuated the increased BMD loss over time observed in individuals with long diabetes durations ( $>4$ years) [87]. In addition, two studies reported that the effect of T2DM on fracture risk was diminished or absent in obese individuals $[115,118]$. Taken together, these results suggest a protective effect of high BMI, possibly mediated by a beneficial increase in BMD. Alternatively, there may be a protective effect of increased padding of bone tissues in obese individuals.

Nakamura et al. observed that elderly subjects with T2DM and reduced handgrip strength exhibited diminished cortical thickness but no changes in trabecular VBMD [82]. This result indicates that reduced muscle strength, or sarcopenia, is an important factor associated with cortical thinning and, consequently, with increased fracture risk. 


\section{Discussion}

The present review provides data on bone health in the elderly with T2DM. However, as most of the studies included were epidemiological in nature, an inevitable uncertainty remains with regard to parameters such as diabetes status and type. Indeed, a few studies attempted to quantify the degree of certainty regarding the type of diabetes present in their subjects. These estimates of certainty appeared to be sufficiently high ( $\geq 97 \%)[87,102]$. In case of contamination of the included studies with individuals having T1DM, underestimation of BMD and overestimation of fracture risk are expected [13]. This is supported by the findings reported in two of the included studies, in which comparisons between individuals with T1DM and individuals with T2DM were also performed $[53,124]$. In the study conducted by Tuominen et al., the individuals with T1DM exhibited lower aBMD values compared to those with T2DM and those without DM [53]. These findings were consistent with those of previous meta-analyses $[13,144]$ and were neither explained by differences in BMI nor by insulin treatment. Wallander et al. reported that fracture risks (any, hip, major osteoporotic, and ankle) were higher in individuals with T1DM compared to any subgroup of individuals with T2DM [124].

The majority of studies examining OC, P1NP, and CTX/NTX indicated suppression of bone turnover mediated by the presence, severity, and duration of T2DM. This suppression appears to be correlated with the presence and severity of diabetes. Owing to relatively small sample sizes, most of the studies reported unadjusted results and performed few subgroup analyses in general. Therefore, it is not possible to distinguish, for instance, the sex-specific effects. However, there are no clear differences between the results of studies examining one sex and those examining the other or both sexes. The insufficiency of evidence along with varying proxies for disease severity (e.g., comorbidities or glycemic control) do not allow exact conclusions regarding the specific effects of disease severity and comorbidities.

The findings regarding vitamin D and PTH suggest that the levels of vitamin D or PTH might be affected in individuals with T2DM, although the evidence is insufficient, and further research is warranted.

Reduced bone turnover may lead to the accumulation of microfractures and impaired bone quality, resulting in reduced adaptability to alterations in mechanical stress. Furthermore, reduced bone turnover is expected to lead to a relative increase in bone mineral content due to reduced BMD loss over time.

As a consequence of reduced bone turnover, it appears that individuals with T2DM do indeed exhibit a higher aBMD compared to those without DM. Studies on bone microarchitecture suggest compromised bone integrity with more trabecular bone and diminished cortical bone, which correlates highly to bone quality [145] and may indicate reduced bending strength and axial loadbearing capacity. In addition, some studies reported that T2DM was associated with a greater aBMD loss over time, possibly indicating a subgroup effect, warranting further research.

The difference in aBMD between individuals with T2DM and those without T2DM appeared to be more pronounced in women than in men, although this conclusion must be made with caution due to the considerable heterogeneity of the studies. Overall, no clear demographic variation in the effect of T2DM on bone structure was observed.

These alterations in bone structure reduce bone strength and increase fracture risk, which is observed particularly in the cases of hip and overall fracture risk. The evidence for an increased 
risk of vertebral fracture presented here is inconsistent and warrants further research. However, a larger proportion of studies examining hip-related regions (total hip, femoral neck, and trochanteric region) reported increases in aBMD compared to studies examining vertebral sites. These differential $\mathrm{aBMD}$ alterations between skeletal regions may be related to differences in their respective cortical and trabecular compartments and might affect the site-specific fracture risk. Thus, this may represent a smaller degree of pathology in vertebral bone, which would in turn lead to a less pronounced increase in fracture risk, if any. In addition, there may be a larger effect of under-reporting in vertebral fractures, as many of these fractures do not present with any symptoms and require imaging for verification.

Under-reporting is a general concern in registry studies, where both fracture rates and T2DM prevalence/incidence may be subject to error, in both cases leading to underestimation of associations.

In the present review, no statistical analyses were performed. Therefore, the heterogeneity of the studies could not be quantified, and the overall size and significance of the effects on bone health could not be estimated. Furthermore, owing to the observational nature of almost all the articles included in the present review, causality could not be determined. However, in the cases of all three areas of interest-bone structure, fracture risk, and bone turnover-clear trends were observed indicating the presence of reduced bone turnover along with altered bone distribution and a concurrent increase in fracture risk in elderly individuals with T2DM.

Fracture risk in individuals with T2DM may, however, be influenced by a variety of factors which have not or have only slightly been touched upon in the present review. These factors are discussed in the following.

None of the included articles examined the levels and the effects of advanced glycation end products (AGEs). AGEs are proteins or lipids that have become glycated as a result of exposure to sugars [146]. AGEs are more abundant in tissues in T2DM and have been shown to affect bone biomechanical properties due to cross-linking with bone proteins [147]. This process occurs more freely in low states of bone turnover, as matrix proteins are exposed to the environment for an extended time. In turn, AGEs increase oxidative stress and inflammation, besides negatively affecting bone turnover [147].

T2DM is a condition of low-grade inflammation [148, 149], a process that is closely (and possibly reversibly) linked to impaired bone turnover in several conditions [150, 151].

Besides bone fragility, an increased risk of falls would result in a consequent increase in fracture risk. Each year, approximately $30 \%$ of individuals aged over 65 years experience a fall [152], and there is evidence that fall risk is further increased in older adults with T2DM [153].

Fall risk may in turn be influenced by a variety of risk factors. De Mettelinge et al. reported polypharmacy, poor walking performance, and reduced cognitive function to be mediators of falls in diabetes [154]. Furthermore, characteristics of posture and gait have been demonstrated to be affected in individuals with T2DM and a history of falls [155].

Gait performance may be affected by disorders affecting proprioception (diabetic sensory neuropathy) [102, 107, 121], visual acuity (diabetic retinopathy) [92, 94, 121, 125], or muscle strength [82], each of which has been reported to be associated with fracture risk.

Other significant contributors to falls in the elderly in general-and perhaps in individuals with diabetes in particular-are dizziness, orthostatic hypotension, antihypertensive drug use, and concomitant cardiovascular disease [102, 127, 156, 157]. 
Finally, fall risk is closely associated with the risk of hypoglycemia, e.g., due to use of insulin and sulfonylureas. Indeed, almost all the included studies reported the highest increase in fracture risk among those treated with insulin, and two studies reported no residual effect when adjusting for insulin use [124, 129].

However, it is difficult to elucidate the full nature of these associations, as polypharmacy and use of insulin represent more severe cases of T2DM with longer diabetes duration and a higher comorbid load, allowing an increased impact on bone turnover and quality.

In relation to all the topics reviewed, conflicting results have been reported. This may be due to a variety of factors, including limited sample sizes and the considerable amount of both known and unknown possible confounders. Certain subject characteristics are fundamentally linked; therefore, the effects of individual characteristics are difficult to account for. This may be the case for glucose-lowering drugs and therapies for comorbidities (and the duration of treatment), which may exert direct effects on bone biochemical and biomechanical properties and also be inherently linked to the severity and duration of T2DM along with the presence of complications and, for certain drugs, hypoglycemic episodes. Other relevant confounders that were not investigated are low-grade inflammation and, in the case of NTX, renal function.

In conclusion, health problems in the elderly, particularly those with T2DM, are numerous, and the elevated risk of fractures results in increased morbidity and mortality. The increased fracture risk may present an even bigger challenge in individuals with T2DM, as Tebé et al. reported that mortality following hip fracture was higher in the elderly with T2DM compared to those without DM [100]. In this context, considering that common prediction tools underestimate fracture risk in diabetes [12], the development of more sensitive diagnostic tools for evaluating bone health in the elderly with T2DM is essential.

The use of newer bone imaging techniques, such as HRpQCT, is limited to the research setting and awaiting studies to demonstrate their clinical utility, while the use of TBS appears to be a more validated and easily accessible method for the evaluation of bone quality.

Further research exploring underlying mechanisms for diabetes-related effects on bones is required. In particular, more intervention trials are required to establish causality and determine reversibility of the effects on bone biochemical and biomechanical properties. 
Table 1 Overview of findings related to biochemical markers of bone in type 2 diabetes mellitus.

\begin{tabular}{|c|c|c|c|c|c|}
\hline Ref & Author & $\mathbf{n}=$ & Population Characteristics & Adjusted for & Findings \\
\hline \multicolumn{6}{|c|}{ Randomized Controlled Trials } \\
\hline [47] & Bulló et al. & $\begin{array}{l}79 \\
\text { (38 T2DM; } \\
41 \text { non-DM) }\end{array}$ & $\begin{array}{l}\text { Age: Mean } 68.5 \\
\text { Spanish } \\
\text { Men }\end{array}$ & $\begin{array}{l}\text { BMI, physical activity, } \\
\text { intervention group, use of } \\
\text { statins, presence of T2DM, } \\
\text { values of the dependent } \\
\text { variable at baseline }\end{array}$ & $\begin{array}{l}\text { Subjects not taking oral antidiabetics: } \\
\text { Baseline OC positively correlated to FPI, HOMA-IR, and } \\
\text { HOMA- } ß \text { after } 2 \text { yr. follow-up } \\
\text { Changes (during follow-up) in total OC associated with: } \\
\text { - Increase in HOMA- } \text { (insulin production) } \\
\text { - No effect on FPG, FPI, and HOMA-IR } \\
\text { Changes (during follow-up) in ucOC associated with: } \\
\text { - Decrease in HOMA-IR (insulin resistance) } \\
\text { - No effect on FPG, FPI and HOMA- } ß\end{array}$ \\
\hline \multicolumn{6}{|c|}{ Prospective Cohort Studies } \\
\hline$[46]$ & $\begin{array}{l}\text { Rianon et } \\
\text { al. }\end{array}$ & $\begin{array}{l}69 \\
\text { All w/T2DM }\end{array}$ & $\begin{array}{l}\text { Age: } \geq 50 \text { year } \\
\text { Mexican American } \\
71 \% \text { women }\end{array}$ & $\begin{array}{l}\text { Age groups, BMI, femoral } \\
\text { neck BMD, serum creatinine, } \\
\text { calcium, } 25(\mathrm{OH}) \mathrm{D} \text {, diabetes } \\
\text { duration }\end{array}$ & $\begin{array}{l}\text { Higher } \mathrm{HbA} 1 \mathrm{c}(>8) \text { correlated with: } \\
\text { - Lower OC in men } \geq 65 \text { years } \\
\text { - No effect on sclerostin in men. } \\
\text { - No effect in women (nonsignificant Increase in sclerostin, } p= \\
0.07) . \\
\text { Longer disease duration: } \\
\text { - No effect on sclerostin or OC }\end{array}$ \\
\hline [29] & Yeap et al. & $\begin{array}{l}\text { 2,966 } \\
\text { (445 T2DM; } \\
\text { 2,521 non-DM) }\end{array}$ & $\begin{array}{l}\text { Age: } 70-89 \\
\text { Australian } \\
\text { Men }\end{array}$ & $\begin{array}{l}\text { Age, smoking, BMI, WHR, } \\
\text { hypertension, dyslipidemia, } \\
\text { creatinine, vitamin D, } \\
\text { Charlson Comorbidity Index }\end{array}$ & $\begin{array}{l}\text { T2DM associated with: } \\
\text { - Lower TOC, UCOC, P1NP, and CTX } \\
\text { - Higher ratio of ucOC to TOC } \\
\text { For every } 1 \text { SD increase in each bone turnover marker, there } \\
\text { was a } 36-45 \% \text { reduction in the risk of prevalent diabetes }\end{array}$ \\
\hline
\end{tabular}




\begin{tabular}{|c|c|c|c|c|c|}
\hline [31] & $\begin{array}{l}\text { Dobnig et } \\
\text { al. }\end{array}$ & $\begin{array}{l}\text { 1,664 } \\
\text { (583 T2DM; } \\
\text { 1,081 non-DM) }\end{array}$ & $\begin{array}{l}\text { Age: } \geq 70 \\
\text { Austrian } \\
\text { Women } \\
\text { Nursing home residents }\end{array}$ & $\begin{array}{l}\text { Age, weight, mobility score, } \\
\text { creatinine clearance }\end{array}$ & $\begin{array}{l}\text { T2DM associated with: } \\
\text { - Lower OC, CTX, and PTH } \\
\text { - No effect on vitamin D } \\
\text { HbA1C negatively correlated with OC and CTX. } \\
\text { The slopes of the declines in OC and CTX were steeper in } \\
\underline{\text { T2DM. }}\end{array}$ \\
\hline [32] & $\begin{array}{l}\text { Gerdhem et } \\
\text { al. }\end{array}$ & $\begin{array}{l}\text { 1,132 } \\
\text { (74 T2DM; } \\
\text { 1,058 non-DM) }\end{array}$ & $\begin{array}{l}\text { Age: All } 75 \\
\text { Swedish } \\
\text { Women }\end{array}$ & Weight, $\mathrm{p}$-creatinine & $\begin{array}{l}\text { T2DM associated with: } \\
\text { - Lower OC, CTX, and vitamin D } \\
\text { - (Lower) U-DPD/crea (nonsignificant when adjusted) } \\
\text { - No effect on bone ALP or PTH }\end{array}$ \\
\hline$[45]$ & $\begin{array}{l}\text { Hannemann } \\
\text { et al. }\end{array}$ & $\begin{array}{l}498 \\
\text { (65 T2DM; } \\
433 \text { non-DM) }\end{array}$ & $\begin{array}{l}\text { Age: Mean } 62.0 \\
\text { German } \\
\text { Postmenopausal women }\end{array}$ & Unadjusted & $\begin{array}{l}\text { T2DM associated with: } \\
\text { - Lower OC }\end{array}$ \\
\hline \multicolumn{6}{|c|}{ Case-control Studies } \\
\hline [23] & Sahin et al. & $\begin{array}{l}99 \\
\text { (47 T2DM; } \\
52 \text { non-DM) }\end{array}$ & $\begin{array}{l}\text { Age: Mean 60.0-61.8 } \\
\text { Turkish } \\
\text { Postmenopausal women }\end{array}$ & Unadjusted & $\frac{\text { T2DM not associated with: }}{\text { - OC, CTX, PTH or ALP }}$ \\
\hline \multicolumn{6}{|c|}{ Cross-sectional Studies } \\
\hline [26] & Liu et al. & $\begin{array}{l}775 \\
\text { (388 T2DM; } \\
245 \text { IGM; } \\
142 \text { NGM) }\end{array}$ & $\begin{array}{l}\text { Age: }>50 \text { years (mean } 73.5- \\
76.7) \\
\text { Han Chinese } \\
\text { Men }\end{array}$ & Serum creatinine, age, BMI & $\begin{array}{l}\text { T2DM associated with: } \\
\text { - Lower ß-CTX, OC, and P1NP } \\
\text { - Unaffected ALP, 25(OH)D, and PTH } \\
\text { FPG negatively correlated with OC }\end{array}$ \\
\hline [42] & $\begin{array}{l}\text { Mitchell et } \\
\text { al. }\end{array}$ & $\begin{array}{l}4,713 \\
\text { (325 T2DM; } \\
797 \text { IFG; } \\
\text { 3,591 NFG) }\end{array}$ & $\begin{array}{l}\text { Age: Mean } 68 \\
\text { Swedish } \\
\text { Women }\end{array}$ & $\begin{array}{l}\text { FPG/FPI, age, height, BMI, } \\
\text { smoking status, physical } \\
\text { activity, education }\end{array}$ & $\begin{array}{l}\text { T2DM associated with: } \\
\text { - Lower OC and sclerostin } \\
\text { IFG associated with: } \\
\text { - Lower OC } \\
\text { FPG inversely correlated with OC and sclerostin. } \\
\text { FPI inversely correlated with OC. }\end{array}$ \\
\hline
\end{tabular}




\begin{tabular}{|c|c|c|c|c|c|}
\hline [37] & $\begin{array}{l}\text { Maagensen } \\
\text { et al. }\end{array}$ & $\begin{array}{l}33 \\
\text { (8 T2DM; } \\
8 \text { NAFLD + T2DM; } \\
8 \text { NAFLD + NGT; } \\
9 \text { NGT) }\end{array}$ & $\begin{array}{l}\text { Age: Means } 55.5,65.0,58.5 \text {, } \\
54.0 \\
\text { Danish } \\
45.5 \% \text { women }\end{array}$ & Unadjusted & $\begin{array}{l}\text { Regardless of NAFLD status, T2DM is associated with } \\
\text { (compared to controls): } \\
\text { - Lower OC and P1NP } \\
\text { - No change in CTX } \\
\text { - Attenuated suppression of CTX } \\
\text { - No change in the suppression of OC or P1NP }\end{array}$ \\
\hline [35] & Zhang et al. & $\begin{array}{l}408 \\
\text { All w/T2DM }\end{array}$ & $\begin{array}{l}\text { Age: } 55-70 \\
\text { Chinese } \\
64.7 \% \text { women }\end{array}$ & Unadjusted & $\begin{array}{l}\text { Diabetic retinopathy associated with: } \\
\text { - Lower ß-CTX } \\
\text { - Unaltered 25(OH)D, P1NP, AZGP1, FGF21, and Osteonectin } \\
\text { Diabetic macular edema associated with: } \\
\text { - Lower P1NP and ß-CTX } \\
\text { - Unaltered 25(OH)D, AZGP1, FGF21, and Osteonectin }\end{array}$ \\
\hline [27] & Shou et al. & $\begin{array}{l}1,316 \\
373 \text { T2DM; } \\
943 \text { non-DM) }\end{array}$ & $\begin{array}{l}\text { Age: } \geq 80 \\
\text { Chinese } \\
\text { Men }\end{array}$ & Unadjusted & $\begin{array}{l}\text { T2DM associated with: } \\
\text { - Lower OC, P1NP, and B-CTX } \\
\text { Also found negative linear associations }(\mathrm{p}<0.01) \text { between all } \\
\text { turnover marker levels and all measures of BMD. }\end{array}$ \\
\hline [38] & Raška et al. & $\begin{array}{l}283 \\
(112 \text { T2DM; } \\
171 \text { non-DM) }\end{array}$ & $\begin{array}{l}\text { Age: Mean 64.0-65.6 } \\
\text { Czech } \\
\text { Postmenopausal women }\end{array}$ & Unadjusted & $\begin{array}{l}\text { T2DM associated with: } \\
\text { - Lower OC and } 25(\mathrm{OH}) \mathrm{D} \\
\text { No difference in ß-CTX, P1NP, or sclerostin }\end{array}$ \\
\hline [36] & Osima et al. & $\begin{array}{l}443 \\
(22 \text { T2DM; } \\
421 \text { non-DM) }\end{array}$ & $\begin{array}{l}\text { Age: } 54-94 \\
\text { Norwegian } \\
\text { Postmenopausal women }\end{array}$ & Age, fracture status. & $\begin{array}{l}\text { T2DM associated with: } \\
\text { - Lower vitamin D } \\
\text { - No effect on P1NP, CTX, or PTH } \\
\text { Increasing glucose associated with: } \\
\text { - Lower P1NP and CTX } \\
\text { Increasing insulin and insulin resistance associated with: } \\
\text { - Lower CTX } \\
\text { - No effect on P1NP }\end{array}$ \\
\hline [39] & Iki et al. & $\begin{array}{l}\text { 1,683 } \\
\text { (313 T2DM; } \\
\text { 1,370 non-DM) }\end{array}$ & $\begin{array}{l}\text { Age: } 72.9 \text { mean } \\
\text { Japanese } \\
\text { Men }\end{array}$ & Unadjusted & $\begin{array}{l}\text { T2DM associated with: } \\
\text { - Lower OC, P1NP, and PTH } \\
\text { - No effect on TRACP5b or CTX }\end{array}$ \\
\hline
\end{tabular}




\begin{tabular}{|c|c|c|c|c|c|}
\hline [28] & Furst et al. & $\begin{array}{l}35 \\
(16 \text { T2DM; } \\
19 \text { non-DM) }\end{array}$ & $\begin{array}{l}\text { Age: Mean 65.4-65.6 } \\
\text { US Citizens } \\
\text { Postmenopausal women }\end{array}$ & Unadjusted & $\begin{array}{l}\text { T2DM associated with: } \\
\text { - Lower P1NP and CTX } \\
\text { - No effect on ALP, PTH, or 25(OH)D }\end{array}$ \\
\hline [43] & Yano et al. & $\begin{array}{l}1,870 \\
\text { (182 T2DM; } \\
1,688 \text { non-DM) }\end{array}$ & $\begin{array}{l}\text { Age: } \geq 50 \text { (mean 68.9) } \\
\text { Japanese } \\
58.6 \% \text { women }\end{array}$ & Unadjusted & $\begin{array}{l}\text { T2DM associated with: } \\
\text { - Lower ucOC } \\
\text { - Lower TRACP5b in women but not in men } \\
\text { Increasing HbA1c, FPG, and insulin associated with: } \\
\text { - Decreasing UcOC } \\
\text { - No effect on TRACP5b } \\
\text { Increasing HOMA-IR: } \\
\text { - No effect on UCOC or TRACP5b }\end{array}$ \\
\hline [49] & $\begin{array}{l}\text { Feldbrin et } \\
\text { al. }\end{array}$ & $\begin{array}{l}100 \\
\text { (33 T2DM + HTN; } \\
39 \text { HTN: } \\
28 \text { healthy) }\end{array}$ & $\begin{array}{l}\text { Age: Mean } 59.8-62.7 \\
\text { Israeli } \\
55 \% \text { women } \\
\text { All: Hypertension }\end{array}$ & Unadjusted & $\begin{array}{l}\text { T2DM associated with: } \\
\text { - Lower P1NP } \\
\text { HbA1C and FPG correlated with: } \\
\text { - Decreasing P1NP } \\
\text { HOMA-ß correlated with: } \\
\text { - Increasing P1NP } \\
\text { HOMA-IR and OPG not associated with anything }\end{array}$ \\
\hline [30] & $\begin{array}{l}\text { Yamamoto } \\
\text { et al. }\end{array}$ & $\begin{array}{l}495 \\
\text { (255 T2DM; } \\
240 \text { non-DM) }\end{array}$ & $\begin{array}{l}\text { Age: Mean 63.1-71.2 } \\
\text { Japanese } \\
63 \% \text { women }\end{array}$ & Unadjusted & $\begin{array}{l}\text { T2DM associated with: } \\
\text { - Lower ß-CTX, OC, P1NP, and PTH } \\
\text { - Higher ALP and vitamin D } \\
\text { In both sexes }\end{array}$ \\
\hline [48] & Xia et al. & $\begin{array}{l}110 \\
\text { (70 T2DM; } \\
40 \text { non-DM) }\end{array}$ & $\begin{array}{l}\text { Age: Mean 74.3-78.1 } \\
\text { Chinese } \\
\text { Men }\end{array}$ & Unadjusted & $\begin{array}{l}\text { T2DM and albuminuria associated with: } \\
\text { - Lower OC (declining with higher albuminuria) } \\
\text { - Higher PTH (increasing with higher albuminuria) } \\
\text { - No effect on ALP }\end{array}$ \\
\hline
\end{tabular}




\begin{tabular}{|c|c|c|c|c|c|}
\hline [50] & Rasul et al. & $\begin{array}{l}120 \\
\text { All w/T2DM }\end{array}$ & $\begin{array}{l}\text { Age: Means } 61-66 \\
\text { Austrian } \\
40.1 \% \text { women }\end{array}$ & Unadjusted & $\begin{array}{l}\text { Polyneuropathy associated with: } \\
\text { - Higher OC and CTX in men } \\
\text { - No effect on OC or CTX in women/total } \\
\text { - Higher P1NP in men and total } \\
\text { - No effect on P1NP in women } \\
\text { - No effect on ALP, vitamin D, or PTH }\end{array}$ \\
\hline [44] & Iki et al. & $\begin{array}{l}\text { 1,597 } \\
\text { (286 T2DM; } \\
\text { 1,311 non-DM) }\end{array}$ & $\begin{array}{l}\text { Age: Mean } 73.0 \\
\text { Japanese } \\
\text { Men }\end{array}$ & $\begin{array}{l}\text { Age, height, weight, weekly } \\
\text { alcohol consumption, smoking } \\
\text { (pack-years), physical activity, } \\
\text { milk intake, fermented } \\
\text { soybean product intake }\end{array}$ & $\begin{array}{l}\text { Fasting glucose, fasting insulin, } \mathrm{HOMA}-\mathrm{IR}, \mathrm{HbA} 1 \mathrm{C} \text { and T2DM } \\
\text { prevalence inversely correlated with: } \\
\text { - iOC (only when unadjusted for } \mathrm{ucOC} \text { ) } \\
\text { - ucOC (also when adjusted for } \mathrm{iOC)} \\
\text { - Ratio of ucOC to iOC } \\
\text { Fasting insulin inversely correlated with TRACP5b }\end{array}$ \\
\hline [51] & Bulló et al. & $\begin{array}{l}251 \\
\text { (110 T2DM; } \\
141 \text { non-DM) }\end{array}$ & $\begin{array}{l}\text { Age: Mean } 67.70-67.82 \\
\text { Spanish } \\
50.6 \% \text { women }\end{array}$ & Unadjusted & $\begin{array}{l}\text { T2DM associated with: } \\
\text { - Higher OPG } \\
\text { - Lower DPD } \\
\text { - No effect on vitamin D, PTH, or bone ALP }\end{array}$ \\
\hline [41] & Zhou et al. & $\begin{array}{l}\text { 1,579 } \\
\text { (890 T2DM; } \\
689 \text { non-DM) }\end{array}$ & $\begin{array}{l}\text { Age: Mean } 56.1-58.5 \\
\text { Chinese } \\
\text { Women }\end{array}$ & Unadjusted & $\begin{array}{l}\text { T2DM associated with: } \\
\text { - Higher urinary NTX secretion } \\
\text { - Lower OC } \\
\text { - No effect on PTH, ALP, or calcitonin } \\
\text { Regardless of BMI group }\end{array}$ \\
\hline [40] & $\begin{array}{l}\text { Chailurkit et } \\
\text { al. }\end{array}$ & $\begin{array}{l}163 \\
\text { (54 T2DM; } \\
109 \text { non-DM) }\end{array}$ & $\begin{array}{l}\text { Age: } 50-88 \text { (mean 62.8-66.9) } \\
\text { Thai } \\
\text { Postmenopausal women }\end{array}$ & Age, BMI & $\begin{array}{l}\text { T2DM associated with: } \\
\text { - Attenuated suppression of CTX after oral glucose } \\
\text { - No suppression of OPG after oral glucose } \\
\text { - No effect on baseline CTX or OPG }\end{array}$ \\
\hline [22] & $\begin{array}{l}\text { Dennison et } \\
\text { al. }\end{array}$ & $\begin{array}{l}909 \\
\text { (65 T2DM; } \\
844 \text { non-DM) }\end{array}$ & $\begin{array}{l}\text { Age: Mean 84.6-66.4 } \\
\text { UK Citizens } \\
48.8 \% \text { women }\end{array}$ & Unadjusted & $\frac{\text { T2DM not associated with: }}{\text { - OC or sclerostin }}$ \\
\hline
\end{tabular}




\begin{tabular}{|c|c|c|c|c|c|}
\hline [34] & $\begin{array}{l}\text { Ardawi et } \\
\text { al. }\end{array}$ & $\begin{array}{l}964 \\
\text { (482 T2DM; } \\
482 \text { non-DM) }\end{array}$ & $\begin{array}{l}\text { Age: Mean } 59.60 \pm 7.90 \\
\text { Saudi Arabians } \\
\text { Postmenopausal women }\end{array}$ & $\begin{array}{l}\text { Age, BMI, HbA1c, vitamin D, } \\
\text { BMD }\end{array}$ & $\begin{array}{l}\text { T2DM associated with: } \\
\text { - Increased sclerostin } \\
\text { - Reduced OC, P1NP, CTX, and urinary NTX } \\
\text { - Lower PTH and IGF-1 }\end{array}$ \\
\hline [33] & $\begin{array}{l}\text { García- } \\
\text { Martín et al. }\end{array}$ & $\begin{array}{l}124 \\
\text { (74 T2DM; } \\
50 \text { non-DM) }\end{array}$ & $\begin{array}{l}\text { Age: } 57.7 \pm 6.5 \\
\text { Spanish } \\
52 \% \text { women }\end{array}$ & Unadjusted & $\begin{array}{l}\text { T2DM associated with: } \\
\text { - Higher sclerostin } \\
\text { - Lower CTX } \\
\text { - Lower TRAP5b } \\
\text { - Unaffected OC and vitamin D } \\
\text { Sclerostin (pmol/L) linearly correlated with: } \\
\text { - Duration of T2DM (years): } r=0.238, p=0.044 \\
\text { - Not significantly with HbA1c }(\%): r=0.200, p=0.09\end{array}$ \\
\hline
\end{tabular}

25(OH)D: 25-hydroxy-vitamin D, ALP: alkaline phosphatase, AZGP1: zinc-binding alpha-2-glycoprotein 1, BMD: bone mineral density, BMI: body mass index, crea: creatinine, CTX: carboxy-terminal collagen crosslinks, DM: diabetes mellitus, T2DM: type 2 DM, DPD: deoxypyridinoline, FGF21: fibroblast growth factor 21, FPG: fasting plasma glucose, FPI: fasting plasma insulin, HbA1c: hemoglobin A1c, HOMA: homeostatic model assessment, HOMA-IR: HOMA for insulin resistance, HOMA- $\beta$ : HOMA-beta-cell, HTN: hypertension, IFG: impaired fasting glucose, IGF-1: insulin-like growth factor 1, OC: osteocalcin, IOC: intact OC, TOC: total OC, ucOC: undercarboxylated OC, NAFLD: non-alcoholic fatty liver disease, NTX: amino-terminal collagen crosslinks, OPG: osteoprotegerin, P1NP: procollagen type $1 \mathrm{n}$-terminal propeptide, PTH: parathyroid hormone, SD: standard deviation, TRACP5b: tartrate-resistant acid phosphatase 5b, U-DPD/crea: urine-DPD/creatinine-ratio, WHR: waist-hip ratio. 
Table 2 Overview of findings related to structural markers in type 2 diabetes mellitus.

\begin{tabular}{|c|c|c|c|c|c|c|}
\hline Ref. & Author & $\mathbf{n}=$ & Population & Measurement & Adjusted for & Findings \\
\hline \multicolumn{7}{|c|}{ Randomized Controlled Trials } \\
\hline$[52]$ & $\begin{array}{l}\text { Schwartz et } \\
\text { al. }\end{array}$ & $\begin{array}{l}107 \\
\text { (3,655 intensive } \\
\text { glycemia; } \\
\text { 3,632 standard } \\
\text { glycemia) } \\
\text { All w/T2DM. }\end{array}$ & $\begin{array}{l}\text { Age: } 62.5 \pm 6.7 . \\
\text { US and Canadian } \\
34.6 \% \text { women } \\
\text { All: T2DM and history of, } \\
\text { subclinical evidence of, or } \\
\underline{\text { significant risk factors for CVD }}\end{array}$ & $\begin{array}{l}\text { DXA aBMD: spine, } \\
\text { hip, whole body. }\end{array}$ & $\begin{array}{l}\text { Baseline BMD, age, sex, } \\
\text { race, DXA site, } \\
\text { comorbidity }{ }^{\mathrm{a}} \text {, medication }{ }^{\mathrm{a}} \text {, } \\
\text { trial interventiona. }\end{array}$ & $\begin{array}{l}\text { Intensive glycemic control (vs. standard } \\
\text { glycemic control): } \\
\text { - No difference in aBMD change at any site }\end{array}$ \\
\hline \multicolumn{7}{|c|}{ Prospective Cohort Studies } \\
\hline [90] & Iki et al. & $\begin{array}{l}1,951 \\
\text { (200 T2DM; } \\
\text { 1,751 non-DM) }\end{array}$ & $\begin{array}{l}\text { Age: } \geq 65 \\
\text { Japanese } \\
\text { Men } \\
\text { All: T2DM }\end{array}$ & $\begin{array}{l}\text { DXA aBMD: } \\
\text { hip + lumbar spine }\end{array}$ & Unadjusted & $\begin{array}{l}\text { Higher HbA1c or fasting glucose: } \\
\text { - Higher hip aBMD } \\
\text { - Higher lumbar spine aBMD }\end{array}$ \\
\hline [75] & Napoli et al. & $\begin{array}{l}5,554 \\
\text { (875 T2DM; } \\
4,679 \text { non-DM) }\end{array}$ & $\begin{array}{l}\text { Age: } \geq 65(73.6 \pm 5.6) \\
\text { US } \\
\text { Men }\end{array}$ & $\begin{array}{l}\text { DXA aBMD: Lumbar } \\
\text { spine, total hip, } \\
\text { femoral neck, } \\
\text { trochanter. } \\
\text { QCT vBMD: Lumbar } \\
\text { spine. }\end{array}$ & $\begin{array}{l}\text { Logistic regression: BMI, } \\
\text { eGFR, history of falls, and } \\
\text { prior fracture history. }\end{array}$ & $\begin{array}{l}\text { T2DM: } \\
\text { - Higher spine aBMD. } \\
\text { - Higher integral spine vBMD. }\end{array}$ \\
\hline$[65]$ & $\begin{array}{l}\text { Mitama et } \\
\text { al. }\end{array}$ & $\begin{array}{l}\text { 6,556 } \\
\text { (792 T2DM; } \\
649 \text { IGM; } \\
\text { 5,115 non-DM) }\end{array}$ & $\begin{array}{l}\text { Age: Means } 67.7 \text { (men), } 68.3 \\
\text { (women) } \\
\text { Japanese } \\
57.5 \% \text { women }\end{array}$ & $\begin{array}{l}\text { DXA aBMD: Lumbar } \\
\text { spine }\end{array}$ & $\begin{array}{l}\text { Age, BMD, CRP, eGFR, } \\
\text { albumin, exercise, } \\
\text { smoking, alcohol, family } \\
\text { history of fracture, IHD, } \\
\text { CVD and previous fracture }\end{array}$ & $\begin{array}{l}\text { T2DM: } \\
\text { - Higher aBMD. }\end{array}$ \\
\hline [105] & $\begin{array}{l}\text { Majumdar } \\
\text { et al. }\end{array}$ & $\begin{array}{l}57,938 \\
\text { (8,840 T2DM; } \\
49,098 \text { non-DM) }\end{array}$ & $\begin{array}{l}\text { Age: Means } 67.1 / 63.8 \\
\text { Canadian } \\
\text { Women }\end{array}$ & $\begin{array}{l}\text { DXA aBMD: femoral } \\
\text { neck }\end{array}$ & Unadjusted & $\begin{array}{l}\text { T2DM: } \\
\text { - Higher aBMD at baseline. } \\
\text { T2DM duration: } \\
\text { - No difference in aBMD }\end{array}$ \\
\hline [84] & $\begin{array}{l}\text { Pritchard et } \\
\text { al. }\end{array}$ & $\begin{array}{l}\text { Baseline: } n=60 \\
\text { (30 T2DM) } \\
\text { Complete follow- } \\
\text { up: } n=37 \text { ( } 15\end{array}$ & $\begin{array}{l}\text { Age: } \geq 60 \\
\text { Canadian } \\
\text { Postmenopausal women }\end{array}$ & $\begin{array}{l}\text { DXA aBMD: Lumbar } \\
\text { spine, total hip, } \\
\text { femoral neck. } \\
\text { MRi radius }\end{array}$ & Unadjusted & $\begin{array}{l}\text { T2DM: } \\
\text { - Higher aBMD at all sites. } \\
\text { No change in trabecular microarchitecture } \\
\text { over } 2 \text { year follow-up. }\end{array}$ \\
\hline
\end{tabular}




\begin{tabular}{|c|c|c|c|c|c|c|}
\hline & & T2DM) & & & & \\
\hline$[113]$ & $\begin{array}{l}\text { Schwartz et } \\
\text { al. }\end{array}$ & $\begin{array}{l}\text { 16,885 } \\
\text { (1,969 T2DM; } \\
14,916 \text { non-DM) }\end{array}$ & $\begin{array}{l}\text { Age: } \text { Mean }>73 \text {. } \\
\text { American } \\
56 \% \text { women }\end{array}$ & $\begin{array}{l}\text { DXA aBMD: T-score, } \\
\text { femoral neck }\end{array}$ & Unadjusted & $\begin{array}{l}\text { T2DM: } \\
\text { - Higher femoral neck BMD T-score }\end{array}$ \\
\hline [31] & $\begin{array}{l}\text { Dobnig et } \\
\text { al. }\end{array}$ & $\begin{array}{l}1,664 \\
\text { (583 T2DM; } \\
1,081 \text { non-DM) }\end{array}$ & $\begin{array}{l}\text { Age: }>70 \\
\text { Austrian } \\
\text { Women }\end{array}$ & $\begin{array}{l}\text { QUS: Calcaneus, } \\
\text { radius, proximal } \\
\text { third phalanx. }\end{array}$ & $\begin{array}{l}\text { Age-, weight-, and mobility } \\
\text { score. }\end{array}$ & $\begin{array}{l}\text { T2DM: } \\
\text { - Higher QUS aBMD. }\end{array}$ \\
\hline$[80]$ & $\begin{array}{l}\text { Strotmeyer } \\
\text { et al. }\end{array}$ & $\begin{array}{l}\text { 2,979 (566 T2DM; } \\
177 \text { IFG; } \\
\text { 2,236 NGM) }\end{array}$ & $\begin{array}{l}\text { Age: } 70-79 \\
\text { US (white and black) } \\
51.1 \% \text { women }\end{array}$ & $\begin{array}{l}\text { DXA aBMD: Total } \\
\text { hip. } \\
\text { CT vBMD: L3. }\end{array}$ & $\begin{array}{l}\text { Sex, race, clinic site, } \\
\text { diabetes variables. }\end{array}$ & $\begin{array}{l}\text { T2DM (and w/impaired glucose metabolism): } \\
\text { - Higher hip aBMD } \\
\text { - No difference in vBMD }\end{array}$ \\
\hline [32] & $\begin{array}{l}\text { Gerdhem et } \\
\text { al. }\end{array}$ & $\begin{array}{l}1,132 \\
\text { (74 T2DM; } \\
\text { 1,058 non-DM) }\end{array}$ & $\begin{array}{l}\text { Age: }>75 \\
\text { Swedish } \\
\text { Women }\end{array}$ & $\begin{array}{l}\text { DXA aBMD: lumbar } \\
\text { spine, femoral neck. } \\
\text { QUS calcaneus. }\end{array}$ & Body weight & $\begin{array}{l}\text { T2DM: } \\
\text { - Higher BMD at all sites. } \\
\text { - No difference in bone mass by QUS. }\end{array}$ \\
\hline [123] & $\begin{array}{l}\text { de Liefde et } \\
\text { al. }\end{array}$ & $\begin{array}{l}6,655 \\
\text { (792 T2DM; } \\
5,863 \text { non-DM) }\end{array}$ & $\begin{array}{l}\text { Age: } \geq 55 \text { (mean } 74 \text { ) } \\
\text { Dutch } \\
59.6 \% \text { women }\end{array}$ & $\begin{array}{l}\text { DXA aBMD: lumbar } \\
\text { spine, femoral neck. }\end{array}$ & $\begin{array}{l}\text { Age, sex, BMI, lower-limb } \\
\text { disability, smoking, use of } \\
\text { either loop or thiazide } \\
\text { diuretics at baseline }\end{array}$ & $\begin{array}{l}\text { Prevalent or incident T2DM: } \\
\text { - Higher aBMD at lumbar spine and femoral } \\
\text { neck } \\
\text { Impaired glucose tolerance: } \\
\text { - No difference in aBMD }\end{array}$ \\
\hline [129] & Napoli et al. & $\begin{array}{l}\text { 5,995 } \\
\text { (881 T2DM; } \\
\text { 5,114 non-DM) }\end{array}$ & $\begin{array}{l}\text { Age: } \geq 65 \text {, median } 73.5 \\
\text { US ( } 90 \% \text { white) } \\
\text { Men }\end{array}$ & $\begin{array}{l}\text { DXA aBMD: total } \\
\text { femur. }\end{array}$ & Unadjusted & $\begin{array}{l}\text { T2DM: } \\
\text { - Higher aBMD } \\
\text { Impaired glucose tolerance: same but less } \\
\text { pronounced effect }\end{array}$ \\
\hline [14] & $\begin{array}{l}\text { Schwartz et } \\
\text { al. }\end{array}$ & $\begin{array}{l}\text { 9,548 } \\
\text { (551 T2DM; } \\
\text { 8,997 non-DM) }\end{array}$ & $\begin{array}{l}\text { Age: Mean }>70 \\
\text { US (white) } \\
\text { Women. }\end{array}$ & $\begin{array}{l}\text { DXA aBMD: } \\
\text { proximal femur. } \\
\text { Single photon } \\
\text { absorptiometry } \\
\text { BMD: distal radius, } \\
\text { calcaneus. }\end{array}$ & Unadjusted & $\begin{array}{l}\text { T2DM: } \\
\text { - Higher aBMD at all sites. }\end{array}$ \\
\hline \multicolumn{7}{|c|}{ Retrospective Cohort Studies } \\
\hline [97] & $\begin{array}{l}\text { Baltrusaitis } \\
\text { et al. }\end{array}$ & $\begin{array}{l}36,744 \\
(19,430 \text { T2DM; } \\
17,314 \text { non-DM) }\end{array}$ & $\begin{array}{l}\text { Age: } \geq 65 \\
\text { U.S. Veterans } \\
1.7 \% \text { women }\end{array}$ & $\begin{array}{l}\text { DXA: Osteoporosis } \\
\text { diagnosis }\end{array}$ & Unadjusted & $\begin{array}{l}\text { T2DM: } \\
\text { - Lower prevalence of osteoporosis }\end{array}$ \\
\hline
\end{tabular}




\begin{tabular}{|c|c|c|c|c|c|c|}
\hline & & 4,614 non-DM) & & & & $\begin{array}{l}\text { - Greater aBMD loss at femoral neck } \\
\text { BMD loss at the lumbar spine attenuated in } \\
\text { the obese }\end{array}$ \\
\hline [102] & Lee et al. & $\begin{array}{l}\text { 2,798,309 } \\
\text { (900,402 T2DM; } \\
\text { 1,897;905 non- } \\
\text { DM) }\end{array}$ & $\begin{array}{l}\text { Age: } \geq 65 \text {. } \\
\text { U.S. Veterans } \\
\text { Men } \\
>98 \% \text { of DM was T2DM }\end{array}$ & $\begin{array}{l}\text { DXA aBMD: femoral } \\
\text { neck }\end{array}$ & Unadjusted & $\begin{array}{l}\frac{\text { T2DM: }}{\text { - Higher femoral neck aBMD. }}\end{array}$ \\
\hline$[91]$ & Oei et al. & $\begin{array}{l}4,135 \\
\text { (420 T2DM; } \\
\text { 3,715 non-DM) }\end{array}$ & $\begin{array}{l}\text { Age: } \geq 55 \\
\text { Dutch } \\
59.4 \% \text { women }\end{array}$ & $\begin{array}{l}\text { DXA aBMD: lumbar } \\
\text { spine, femoral neck. } \\
\text { DXA Hip geometry; } \\
\text { cortical thickness. }\end{array}$ & $\begin{array}{l}\text { Sex, age, height, and } \\
\text { weight (and femoral neck } \\
\text { BMD) }\end{array}$ & $\begin{array}{l}\mathrm{HbA} 1 \mathrm{c} \geq 7.5 \% \text { (vs. } \mathrm{HbA} 1 \mathrm{c}<7.5 \%) \text { and } \mathrm{T} 2 \mathrm{DM} \text { : } \\
\text { - Higher aBMD at lumbar spine and femoral } \\
\text { neck } \\
\text { - Thicker cortices and smaller bone diameter } \\
\text { at femoral neck }\end{array}$ \\
\hline [71] & Leslie et al. & $\begin{array}{l}29,407 \\
(2,356 \text { T2DM; } \\
27,051 \text { non-DM) }\end{array}$ & $\begin{array}{l}\text { Age: } 65.4 \pm 9.4 \\
\text { Canadian } \\
\text { Postmenopausal women }\end{array}$ & $\begin{array}{l}\text { DXA aBMD: } \\
\text { lumbar spine, total } \\
\text { hip, femoral neck. } \\
\text { DXA lumbar spine } \\
\text { TBS }\end{array}$ & Unadjusted & $\begin{array}{l}\text { T2DM: } \\
\text { - Lower prevalence of osteoporosis. } \\
\text { - Higher BMD } \\
\text { - Lower lumbar spine TBS }\end{array}$ \\
\hline \multicolumn{7}{|c|}{ Case-control Studies } \\
\hline$[56]$ & Amer et al. & $\begin{array}{l}61 \\
\text { (31 T2DM; } \\
30 \text { non-DM) }\end{array}$ & $\begin{array}{l}\text { Age: } \geq 60 \\
\text { Egyptian } \\
48.4 \% \text { women (in T2DM } \\
\text { group) }\end{array}$ & $\begin{array}{l}\text { DXA aBMD: lumbar } \\
\text { spine, femoral neck. }\end{array}$ & Age-matched & $\begin{array}{l}\text { T2DM: } \\
\text { - No difference in aBMD. }\end{array}$ \\
\hline [23] & Sahin et al. & $\begin{array}{l}99 \\
\text { (47 T2DM; } \\
52 \text { non-DM) }\end{array}$ & $\begin{array}{l}\text { Age: Mean }>60 \\
\text { Turkish } \\
\text { Postmenopausal women. }\end{array}$ & $\begin{array}{l}\text { DXA aBMD: lumbar } \\
\text { spine, total hip, } \\
\text { femoral neck. }\end{array}$ & Unadjusted & $\frac{\text { T2DM: }}{\text { - Higher BMD at all sites. }}$ \\
\hline \multicolumn{7}{|c|}{ Cross-sectional Studies } \\
\hline [88] & Xu et al. & $\begin{array}{l}1,222 \\
\text { All w/T2DM }\end{array}$ & $\begin{array}{l}\text { Age } \geq 50 \text {. } \\
\text { Tianjin, China } \\
53 \% \text { women }\end{array}$ & $\begin{array}{l}\text { DXA aBMD: whole } \\
\text { body. }\end{array}$ & $\begin{array}{l}\text { Age, sex, BMI, smoking, } \\
\text { alcohol, comorbidities }{ }^{\underline{a}} \text {, } \\
\text { medications }{ }^{\underline{a}} \text {, glycemic } \\
\text { control, diabetes duration }\end{array}$ & $\begin{array}{l}\text { Poor glycemic control }(\mathrm{HbA} 1 \mathrm{c} \geq 7.5 \%) \text { : } \\
\text { - Higher OR of osteoporosis in men } \\
\text { HbA1c higher in women with normal aBMD } \\
\text { (not in men) } \\
\text { DM duration: No difference in aBMD }\end{array}$ \\
\hline [69] & $\begin{array}{l}\text { Sakane et } \\
\text { al. }\end{array}$ & $\begin{array}{l}62 \text { (11 T2DM; } \\
25 \text { prediabetes; } \\
26 \text { NGM) }\end{array}$ & $\begin{array}{l}\text { Age: Median } 59 \text { (19-81) } \\
\text { São Paolo. } \\
\text { All: postsurgical } \\
\text { hypoparathyroidism }\end{array}$ & DXA, TBS. & $\begin{array}{l}\text { BMI, glycemic profile, and } \\
\text { densitometric diagnosis. }\end{array}$ & $\begin{array}{l}\overline{\text { T2DM: }} \\
\text { - Lower TBS }\end{array}$ \\
\hline
\end{tabular}




\begin{tabular}{|c|c|c|c|c|c|c|}
\hline & & & $85 \%$ women & & & \\
\hline [26] & Liu et al. & $\begin{array}{l}775 \text { (388 T2DM; } \\
245 \text { IGM; } \\
142 \text { NGM) }\end{array}$ & $\begin{array}{l}\text { Age: } \geq 50 \text {. } \\
\text { Beijing, Han Chinese } \\
\text { Men }\end{array}$ & $\begin{array}{l}\text { DXA aBMD: } \\
\text { Lumbar spine, total } \\
\text { hip, femoral neck. }\end{array}$ & BMI, age & $\begin{array}{l}\text { T2DM: No difference in aBMD. } \\
\text { Glycemic status: No difference in aBMD }\end{array}$ \\
\hline [137] & $\begin{array}{l}\text { Holloway- } \\
\text { Kew et al. }\end{array}$ & $\begin{array}{l}\text { 1,828 (138 T2DM; } \\
418 \text { IGM; } \\
1,272 \text { NGM) }\end{array}$ & $\begin{array}{l}\text { Age: Mean } 67 \text { (T2DM) } \\
\text { Australian. } \\
\text { 46.9\% women }\end{array}$ & $\begin{array}{l}\text { DXA aBMD: } \\
\text { femoral neck, } \\
\text { lumbar spine }\end{array}$ & $\begin{array}{l}\text { Age, weight, height, } \\
\text { mobility, smoking, alcohol, } \\
\text { medication. }\end{array}$ & $\begin{array}{l}\text { T2DM or IGM (compared to normoglycemia): } \\
\text { - Higher aBMD in obese T2DM women } \\
\text { - No difference in men }\end{array}$ \\
\hline [158] & $\begin{array}{l}\text { Dawson- } \\
\text { Hughes et } \\
\text { al. }\end{array}$ & $\begin{array}{l}184 \\
\text { (40 T2DM; } \\
88 \text { prediabetes; } \\
56 \text { NGM) }\end{array}$ & $\begin{array}{l}\text { Age } \geq 55 \text {. } \\
\text { US (white, black, Asian, } \\
\text { Hispanic, one other) } \\
48.9 \% \text { women }\end{array}$ & $\begin{array}{l}\text { DXA aBMD: } \\
\text { lumbar spine, } \\
\text { femoral neck, mid- } \\
\text { tibia. } \\
\text { TBS. } \\
\text { Osteoprobe BMSi. }\end{array}$ & Age, sex, BMI. & $\begin{array}{l}\text { T2DM: } \\
\text { - Higher femoral neck aBMD (in white } \\
\text { individuals) } \\
\text { - Lower BMSi (in black individuals) } \\
\text { - Unaffected TBS } \\
\frac{\text { HbA1c levels: }}{\text { - No difference in BMSi or TBS }}\end{array}$ \\
\hline [142] & Chi et al. & $\begin{array}{l}\text { 7,835 } \\
\text { (1,313 T2DM; } \\
6,522 \text { non-DM) }\end{array}$ & $\begin{array}{l}\text { Age: Means 60-63 (T2DM) } \\
\text { Korean } \\
60 \% \text { women }\end{array}$ & $\begin{array}{l}\text { DXA aBMD: total } \\
\text { femur, trochanter, } \\
\text { intertrochanter, } \\
\text { femoral neck, ward, } \\
\text { lumbar spine, whole } \\
\text { body. }\end{array}$ & Age, BMI & $\begin{array}{l}\text { T2DM: } \\
\text { - Women: Higher aBMD at Ward's triangle } \\
\text { and lumbar spine } \\
\text { - Men: Higher aBMD in lumbar and thoracic } \\
\text { spine } \\
\text { - aBMD as a predictor of T2DM: associated } \\
\text { with increased odds for T2DM }\end{array}$ \\
\hline [54] & Cherif et al. & $\begin{array}{l}81 \\
\text { All w/T2DM }\end{array}$ & $\begin{array}{l}\text { Age: Mean } 58.4 \\
\text { Tunisian } \\
\text { Postmenopausal women } \\
\text { All: Obese }\end{array}$ & $\begin{array}{l}\text { DXA aBMD: lumbar } \\
\text { spine, femoral neck, } \\
\text { total hip. } \\
\text { BMC (content) }\end{array}$ & $\begin{array}{l}\text { Multiple linear regression: } \\
\text { Age, years since } \\
\text { menopause, weight, } \\
\text { height, waist } \\
\text { circumference, BMI, } \\
\text { alkaline phosphatase, body } \\
\text { compositiona }\end{array}$ & $\begin{array}{l}\text { Glycemic status: } \\
\text { - Not correlated to aBMD }\end{array}$ \\
\hline [70] & $\begin{array}{l}\text { Baleanu et } \\
\text { al. }\end{array}$ & $\begin{array}{l}260 \\
\text { (65 T2DM; } \\
195 \text { non-DM) }\end{array}$ & $\begin{array}{l}\text { Age: } 60-85 \\
\text { Belgian } \\
\text { Postmenopausal women }\end{array}$ & $\begin{array}{l}\text { DXA aBMD: lumbar } \\
\text { spine, total hip, } \\
\text { femoral neck. } \\
\text { DXA TBS. }\end{array}$ & $\begin{array}{l}\text { BMI } \\
\text { FRAX-matched } 1: 3\end{array}$ & $\begin{array}{l}\text { T2DM: } \\
\text { - Increased total hip aBMD. } \\
\text { - Reduced TBS (with similar FRAX). }\end{array}$ \\
\hline [136] & Zhong et al. & $\begin{array}{l}2,170 \\
\text { All w/T2DM }\end{array}$ & $\begin{array}{l}\text { Age: } \geq 50 \text { (means } 61-72 \text { ) } \\
\text { Chinese } \\
54.7 \% \text { (postmenopausal) }\end{array}$ & $\begin{array}{l}\text { DXA aBMD: lumbar } \\
\text { spine, total hip and } \\
\text { femoral neck }\end{array}$ & $\begin{array}{l}\text { Age, BMI, diabetic status, } \\
\text { comorbidities } \underline{\text { a }} \text {, sex } \\
\text { hormones, } 25(\mathrm{OH}) \text { vitamin }\end{array}$ & $\begin{array}{l}\text { Presence of microangiopathy: } \\
\text { - Lower aBMD at all sites in women } \\
\text { - No difference in men }\end{array}$ \\
\hline
\end{tabular}




\begin{tabular}{|c|c|c|c|c|c|c|}
\hline & & & women & & D. & \\
\hline [159] & $\begin{array}{l}\text { Valentini et } \\
\text { al. }\end{array}$ & $\begin{array}{l}242 \\
(119 \text { T2DM; } \\
123 \text { non-DM) }\end{array}$ & $\begin{array}{l}\text { Age: } \geq 50 \text { (mean } 74 \text { ) } \\
\text { Italian } \\
\text { Unspecified sex fractions }\end{array}$ & $\begin{array}{l}\text { DXA aBMD, femoral } \\
\text { neck }\end{array}$ & Unadjusted & $\begin{array}{l}\text { T2DM: } \\
\text { - Higher femoral neck aBMD and T-score } \\
\text { Lower FRAX-score: may underestimate the } \\
\text { risk of fracture }\end{array}$ \\
\hline [82] & $\begin{array}{l}\text { Nakamura } \\
\text { et al. }\end{array}$ & $\begin{array}{l}826 \\
(122 \text { T2DM; } \\
704 \text { non-DM) }\end{array}$ & $\begin{array}{l}\text { Age: Mean } 62.3 \text { (T2DM) } \\
\text { Japanese } \\
\text { Women }\end{array}$ & $\begin{array}{l}\text { UL distal radius: } \\
\text { Cortical Thickness, } \\
\text { Trabecular BMD. }\end{array}$ & $\begin{array}{l}\text { Age, BMI, HGS, eGFR, } \\
\text { serum albumin, HbA1c }\end{array}$ & $\begin{array}{l}\text { T2DM: } \\
\text { - Higher trabecular BMD after age } 60 \\
\text { - Lower cortical thickness after age } 40\end{array}$ \\
\hline$[42]$ & $\begin{array}{l}\text { Mitchell et } \\
\text { al. }\end{array}$ & $\begin{array}{l}5,165 \\
\text { (393 T2DM; } \\
947 \text { IFG; } \\
3,825 \text { NGM) }\end{array}$ & $\begin{array}{l}\text { Age: Mean } 82 \text { (men), } 68 \\
\text { (women) } \\
\text { Swedish } \\
\text { 91.2\% women }\end{array}$ & $\begin{array}{l}\text { DXA aBMD: total hip } \\
\text { and femoral shaft. } \\
\text { BMA (cm2) } \\
\text { Femoral neck } \\
\text { diameter }\end{array}$ & $\begin{array}{l}\text { Multiple regression: age, } \\
\text { height, weight, categorical } \\
\text { variables (questionnaires) } \\
\text { smoking, physical activity, } \\
\text { education. }\end{array}$ & $\begin{array}{l}\text { T2DM: } \\
\text { - Higher aBMD. } \\
\text { - Lower BMA at hip and femoral shaft } \\
\text { Similar but less pronounced findings for } \\
\text { individuals with IFG. }\end{array}$ \\
\hline [86] & Jang et al. & $\begin{array}{l}3,383 \\
\text { (644 T2DM; } \\
\text { 1,037 prediabetes; } \\
1,702 \text { NGM) }\end{array}$ & $\begin{array}{l}\text { Age: } \geq 50 \text {. } \\
\text { Korean men }\end{array}$ & $\begin{array}{l}\text { aBMD: lumbar } \\
\text { spine, total hip, } \\
\text { femoral neck }\end{array}$ & $\begin{array}{l}\text { Age, BMI, alcohol, } \\
\text { smoking, serum vitamin } D \text {, } \\
\text { lipid levelsa , hypertension, } \\
\text { physical activity, HOMA-IR }\end{array}$ & $\begin{array}{l}\text { T2DM: } \\
\text { - Higher aBMD at all sites. } \\
\text { aBMD in men with pre-DM were similar to } \\
\text { men with DM in all cases. } \\
\text { T2DM duration > } 5 \text { years: } \\
\text { - Lower femoral neck aBMD }\end{array}$ \\
\hline [76] & $\begin{array}{l}\text { Ho-Pham et } \\
\text { al. }\end{array}$ & $\begin{array}{l}1,729 \\
\text { (137 T2DM; } \\
1,592 \text { non-DM) }\end{array}$ & $\begin{array}{l}\text { Age: Mean } 58.5 \text { (T2DM) } \\
\text { Vietnamese } \\
64.5 \% \text { women }\end{array}$ & $\begin{array}{l}\text { DXA aBMD: Lumbar } \\
\text { spine, total hip, } \\
\text { whole body. } \\
\text { pQCT vBMD: Tibia, } \\
\text { radius. } \\
\text { SSI: Bone strength }\end{array}$ & $\begin{array}{l}\text { Matched: } \\
\text { sex, age, BMI }\end{array}$ & $\begin{array}{l}\text { T2DM: } \\
\text { - Higher aBMD at all sites. } \\
\text { - Higher radial and tibial trabecular vBMD } \\
\text { - Lower bone strength (SSI) } \\
\text { - Near-significant reduced radial cortical } \\
\text { vBMD }\end{array}$ \\
\hline [79] & $\begin{array}{l}\text { de Waard } \\
\text { et al. }\end{array}$ & $\begin{array}{l}608 \\
\text { (98 T2DM; } \\
91 \text { prediabetes; } \\
419 \text { NGM) }\end{array}$ & $\begin{array}{l}\text { Age: Mean } 58 \\
\text { Dutch } \\
51.2 \% \text { women }\end{array}$ & $\begin{array}{l}\text { HRpQCT vBMD } \\
\text { (Trabecular and } \\
\text { Cortical). } \\
\text { Microarchitecture. } \\
\text { Bone strength. }\end{array}$ & $\begin{array}{l}\text { age, sex, BMI, level of } \\
\text { education, smoking, } \\
\text { alcohol, CVD, physical } \\
\text { activity, fracture at or } \\
\text { above the age of 50, } \\
\text { antihyperglycemic } \\
\text { medication. }\end{array}$ & $\begin{array}{l}\text { T2DM w/HbA1c }>7 \%(53 \mathrm{mmol} / \mathrm{mol}): \\
\text { - Lower cortical density at distal radius } \\
\text { - Lower trabecular thickness at distal tibia } \\
\text { T2DM duration }>5 \text { years: } \\
\text { - Higher trabecular number }(\mathrm{Tb} . \mathrm{N}) \text { of the } \\
\text { radius } \\
\text { Pre-DM: } \\
\text { - Only associated with lower Tb.N of the tibia. } \\
\text { - No difference in bone strength. }\end{array}$ \\
\hline
\end{tabular}




\begin{tabular}{|c|c|c|c|c|c|c|}
\hline & & & $\begin{array}{l}\text { Postmenopausal women, } \\
\text { dwelling in high altitudes } \\
(2500 \sim 4500 \mathrm{~m})\end{array}$ & hip, femoral neck & $\begin{array}{l}\text { DM duration, } \\
\text { hypertension, smoking, } \\
\text { pregnancies, systolic blood } \\
\text { pressure, } \mathrm{Hbg} \text {, creatinine, } \\
\text { uric acid, HbAlc. }\end{array}$ & $\begin{array}{l}\text { Age inversely correlated with aBMD/T-score } \\
\text { in femoral neck and hip }\end{array}$ \\
\hline [63] & Sun et al. & $\begin{array}{l}4,080 \\
\text { (906 T2DM; } \\
\text { 3,174 non-DM) }\end{array}$ & $\begin{array}{l}\text { Age: Means 57/59 } \\
\text { Chinese } \\
\text { Postmenopausal women }\end{array}$ & $\begin{array}{l}\text { Quantitative } \\
\text { ultrasound (QUS): } \\
\text { SOS, BUA, stiffness, } \\
\text { T-score }\end{array}$ & $\begin{array}{l}\text { Age, physical activity, } \\
\text { smoking, alcohol } \\
\text { menopause age, BMI }\end{array}$ & $\begin{array}{l}\text { Lower odds for osteopenia with: } \\
\text { - T2DM } \\
- \text { HbA1c }>6.5 \\
\text { - FPG }>7.0 \\
- \text { PPG } \geq 15\end{array}$ \\
\hline [78] & Sheu et al. & $\begin{array}{l}156 \\
\text { (38 T2DM; } \\
118 \text { non-DM) }\end{array}$ & $\begin{array}{l}\text { Age: } \geq 65 \text {, Mean } 80.5 \\
\text { American (+3 African) } \\
\text { Men }\end{array}$ & $\begin{array}{l}\text { MRI: Bone marrow } \\
\text { fat (BMF) } \\
\text { DXA aBMD: lumbar } \\
\text { spine, total hip, } \\
\text { femoral neck. } \\
\text { pQCT vBMD: tibia } \\
\text { and radius. }\end{array}$ & $\begin{array}{l}\text { Age, race, BMI, Leptin, } \\
\text { adiponectin, insulin }\end{array}$ & $\begin{array}{l}\text { T2DM: } \\
\text { - Higher BMF content. } \\
\text { - Higher baseline aBMD at total hip and total } \\
\text { spine } \\
\text { - No difference in vBMD at any site. }\end{array}$ \\
\hline [36] & Osima et al. & $\begin{array}{l}443 \\
\text { (22 T2DM; } \\
421 \text { non-DM) }\end{array}$ & $\begin{array}{l}\text { Age: Means } 70.9 / 68.2 \\
\text { Norwegian } \\
\text { Postmenopausal women }\end{array}$ & CT scan vBMD: Hip. & Age, fracture status. & $\begin{array}{l}\text { T2DM: } \\
\text { - Lower cortical porosity } \\
\text { - Higher total vBMD and cortical vBMD. } \\
\text { Higher glucose levels: } \\
\text { - Lower cortical porosity } \\
\text { Higher total vBMD with higher glucose, insulin } \\
\text { and HOMA-IR }\end{array}$ \\
\hline [39] & Iki et al. & $\begin{array}{l}1,683 \\
\text { (313 T2DM; } \\
\text { 1,370 non-DM) }\end{array}$ & $\begin{array}{l}\text { Age: } \geq 65,72.9 \pm 5.2 \\
\text { Japanese } \\
\text { Men }\end{array}$ & $\begin{array}{l}\text { DXA TBS and aBMD: } \\
\text { Lumbar spine }\end{array}$ & $\begin{array}{l}\text { Age, BMI and aBMD/TBS. } \\
\text { Bone turnover, } \\
\text { pentosidine }\end{array}$ & $\begin{array}{l}\text { Higher FPG, HOMA-IR and HbA1c associated } \\
\text { with: } \\
\text { - Higher aBMD } \\
\text { - Lower TBS }\end{array}$ \\
\hline
\end{tabular}




\begin{tabular}{|c|c|c|c|c|c|c|}
\hline [161] & $\begin{array}{l}\text { Hyassat et } \\
\text { al. }\end{array}$ & $\begin{array}{l}1,079 \\
\text { (530 T2DM; } \\
404 \text { prediabetes; } \\
145 \text { non-DM) }\end{array}$ & $\begin{array}{l}\text { Age: } 61.1 \pm 7.2 \text {. } \\
\text { Jordanian } \\
\text { Postmenopausal women }\end{array}$ & $\begin{array}{l}\text { DXA aBMD: lumbar } \\
\text { spine, femoral neck }\end{array}$ & $\begin{array}{l}\text { Multiple regression: BMI, } \\
\text { diabetes status, family } \\
\text { history of osteoporosis, } \\
\text { physical activity, sun } \\
\text { exposure, dieta, age at } \\
\text { menarche, menopausal } \\
\text { duration, parity }\end{array}$ & $\begin{array}{l}\frac{\text { T2DM: }}{\text { - Lower osteoporosis risk. }}\end{array}$ \\
\hline [55] & Razi et al. & $\begin{array}{l}110 \\
\text { (55 T2DM; } \\
55 \text { non-DM) }\end{array}$ & $\begin{array}{l}\text { Age: Median } 58 \text { (39-79). } \\
\text { Iranian } \\
\text { Postmenopausal women }\end{array}$ & $\begin{array}{l}\text { aBMD: } \\
\text { Lumbar spine, hip, } \\
\text { and subregions. }\end{array}$ & $\begin{array}{l}\text { Age at menopause. } \\
\text { BMI, BMD values at } \\
\text { different sites, s-Bone ALP, } \\
\text { vitamin D, systolic blood } \\
\text { pressure }\end{array}$ & $\begin{array}{l}\frac{\text { T2DM: }}{\text { - No difference in bone mass values. }} \\
\text {. }\end{array}$ \\
\hline [28] & Furst et al. & $\begin{array}{l}35 \\
(16 \mathrm{~T} 2 \mathrm{DM} ; \\
19 \text { non-DM) }\end{array}$ & $\begin{array}{l}\text { Age: Mean } \approx 65.5 \\
\text { US (white) } \\
\text { Postmenopausal women }\end{array}$ & $\begin{array}{l}\text { OsteoProbe BMSi } \\
\text { (bone strength). } \\
\text { DXA aBMD: lumbar } \\
\text { spine, total hip, } \\
\text { femoral neck. } \\
\text { HRpQCT vBMD: } \\
\text { radius, tibia. }\end{array}$ & $\begin{array}{l}\text { Age, nephropathy, } \\
\text { retinopathy, neuropathy, } \\
\text { cardiac disease. }\end{array}$ & $\begin{array}{l}\text { T2DM: } \\
\text { - Reduced BMSi. } \\
\text { - Higher aBMD at femoral neck and total hip. } \\
\text { - Higher trabecular VBMD and stiffness at } \\
\text { radius } \\
\text { - Greater trabecular thickness at radius and } \\
\text { tibia } \\
\text { - No other differences in vBMD or cortical } \\
\text { porosity }\end{array}$ \\
\hline [59] & Cui et al. & $\begin{array}{l}4,988 \\
\text { ( }(\mathrm{D} 2 \mathrm{DM} \text { prevalence } \\
\text { not specified) }\end{array}$ & $\begin{array}{l}\text { Age: Means } 65 \text { (men), } 59 \\
\text { (women) } \\
\text { Chinese } \\
79.3 \% \text { women }\end{array}$ & $\begin{array}{l}\text { DXA aBMD: lumbar } \\
\text { spine. }\end{array}$ & Unadjusted & $\begin{array}{l}\text { Osteoporosis associated with: } \\
\text { - Higher fasting glucose and postprandial } \\
\text { glucose (PPG) } \\
\text { - No effect on HbA1c } \\
\text { T2DM w/PPG > } 7.0 \text { (compared to PPG }<7.0 \text { ): } \\
\text { - Lower aBMD }\end{array}$ \\
\hline [162] & $\begin{array}{l}\text { Siddapur et } \\
\text { al. }\end{array}$ & $\begin{array}{l}60 \\
\text { (30 T2DM; } \\
30 \text { non-DM) }\end{array}$ & $\begin{array}{l}\text { Age: Mean } 59.5 \\
\text { Indian } \\
\text { Postmenopausal women } \\
\text { All: Osteoporosis }\end{array}$ & $\begin{array}{l}\text { DXA aBMD: lumbar } \\
\text { spine. }\end{array}$ & Age-matching. & $\begin{array}{l}\text { T2DM + osteoporosis (vs. Non-DM + } \\
\text { osteoporosis): } \\
\text { - Higher T-score. }\end{array}$ \\
\hline [163] & $\begin{array}{l}\text { Schacter et } \\
\text { al. }\end{array}$ & $\begin{array}{l}34,338 \\
(2,929 \text { T2DM; } \\
28,719 \text { non-DM) }\end{array}$ & $\begin{array}{l}\text { Age: Means } 68.0 \text { (men), } 65.4 \\
\text { (women) } \\
\text { Canadian } \\
92.2 \% \text { women }\end{array}$ & $\begin{array}{l}\text { DXA aBMD: } \\
\text { anteroposterior } \\
\text { spine-hip tissue } \\
\text { thickness. }\end{array}$ & Age, BMI. & $\begin{array}{l}\text { T2DM: } \\
\text { - Higher spine-hip tissue thickness difference } \\
\text { (SHTD) } \\
\text { Greater SHTD associated with higher } \\
\text { likelihood of T2DM. }\end{array}$ \\
\hline
\end{tabular}




\begin{tabular}{|c|c|c|c|c|c|c|}
\hline [164] & Aypak et al. & $\begin{array}{l}275 \\
\text { (66 T2DM; } \\
209 \text { non-DM) }\end{array}$ & $\begin{array}{l}\text { Age: } 72.1+5.4 \\
\text { Turkish } \\
\text { Women }\end{array}$ & $\begin{array}{l}\text { DXA aBMD: lumbar } \\
\text { spine, total hip, } \\
\text { femoral neck, T- } \\
\text { score. }\end{array}$ & Unadjusted & $\begin{array}{l}\text { T2DM: } \\
\text { - Higher femoral neck aBMD. } \\
\text { - Higher osteoporosis rate. }\end{array}$ \\
\hline$[30]$ & $\begin{array}{l}\text { Yamamoto } \\
\text { et al. }\end{array}$ & $\begin{array}{l}495 \\
\text { (255 T2DM; } \\
240 \text { non-DM) }\end{array}$ & $\begin{array}{l}\text { Age: Mean }>60 \text {. } \\
\text { Japanese } \\
63 \% \text { (postmenopausal) } \\
\text { women }\end{array}$ & $\begin{array}{l}\text { DXA aBMD: lumbar } \\
\text { spine, femoral neck. }\end{array}$ & Unadjusted & $\begin{array}{l}\frac{\text { T2DM: }}{\text { - Higher aBMD in women but not in men. }} \\
\text { Highe }\end{array}$ \\
\hline [48] & Xia et al. & $\begin{array}{l}110 \\
\text { (70 T2DM; } \\
40 \text { non-DM) }\end{array}$ & $\begin{array}{l}\text { Age: } \geq 60 \\
\text { Chinese } \\
\text { Men }\end{array}$ & $\begin{array}{l}\text { DXA aBMD: lumbar } \\
\text { spine, total hip, } \\
\text { femoral neck. }\end{array}$ & $\begin{array}{l}\text { Age-matched. } \\
\text { Multivariate regression } \\
\text { controlled for patient } \\
\text { characteristics and } \\
\text { comorbid conditions a }\end{array}$ & $\begin{array}{l}\text { T2DM: } \\
\text { - Lower aBMD at both lumbar spine and } \\
\text { femoral neck. } \\
\text { Osteoporosis associated with increased } \\
\text { albuminuria in T2DM }\end{array}$ \\
\hline$[50]$ & Rasul et al. & $\begin{array}{l}120 \\
\text { All w/T2DM. }\end{array}$ & $\begin{array}{l}\text { Age: Mean }>60 \text {. } \\
\text { Austrian } \\
40.1 \% \text { (postmenopausal) } \\
\text { women }\end{array}$ & $\begin{array}{l}\text { DXA aBMD: lumbar } \\
\text { spine, femoral neck. }\end{array}$ & Sex & $\begin{array}{l}\text { Presence of polyneuropathy: } \\
\text { - No difference in aBMD }\end{array}$ \\
\hline [83] & $\begin{array}{l}\text { Pritchard et } \\
\text { al. }\end{array}$ & $\begin{array}{l}60 \\
\text { (30 T2DM; } \\
30 \text { non-DM) }\end{array}$ & $\begin{array}{l}\text { Age: } \geq 65 \text {. } \\
\text { Canadian } \\
\text { Postmenopausal women }\end{array}$ & $\begin{array}{l}\text { DXA aBMD: lumbar } \\
\text { spine, femoral neck, } \\
\text { total hip. } \\
\text { MRi. }\end{array}$ & $\begin{array}{l}\text { BMI, \% body fat, ethnicity, } \\
\text { age-adjusted Charlson } \\
\text { Index, TUG test result, } \\
\text { total calcium intake, and } \\
\text { total vitamin D intake. }\end{array}$ & $\begin{array}{l}\text { T2DM: } \\
\text { - Higher lumbar spine aBMD (unadjusted) } \\
\text { - Great hole size within trabecular network at } \\
\text { distal radius }\end{array}$ \\
\hline [165] & $\begin{array}{l}\text { Karimifar et } \\
\text { al. }\end{array}$ & $\begin{array}{l}600 \\
\text { (200 T2DM; } \\
400 \text { non-DM) }\end{array}$ & $\begin{array}{l}\text { Age: } \geq 60 \text {. } \\
\text { Iranian } \\
\text { Postmenopausal women }\end{array}$ & $\begin{array}{l}\text { DXA aBMD T-scores: } \\
\text { lumbar spine, } \\
\text { femoral neck }\end{array}$ & $\begin{array}{l}\text { Age, height, weight, BMI, } \\
\text { duration of menopause, } \\
\text { BMD T-score }\end{array}$ & $\begin{array}{l}\text { T2DM: } \\
\text { - No difference in lumbar T-score. } \\
\text { - Lower femoral neck T-score } \\
\text { HbA1c }>7 \% \text { vs. HbA1c } \leq 7 \% \text { : } \\
\text { - No difference in lumbar spine T-score } \\
\text { - Higher femoral neck T-score }\end{array}$ \\
\hline [64] & Shan et al. & $\begin{array}{l}2,447 \\
(1,253 \text { T2DM; } \\
1,194 \text { non-DM) }\end{array}$ & $\begin{array}{l}\text { Age: } 40-80 \text { (mean 60) } \\
\text { Chinese } \\
\text { Women }\end{array}$ & $\begin{array}{l}\text { DXA aBMD: lumbar } \\
\text { spine, femoral neck, } \\
\text { total hip, wards. } \\
\text { Bone projective } \\
\text { area (BPA). }\end{array}$ & $\begin{array}{l}\text { Age, BMI, years since } \\
\text { menopause (YSM), } \\
\text { duration of diabetes }\end{array}$ & $\begin{array}{l}\text { T2DM: } \\
\text { - Higher aBMD at lumbar spine but not hip } \\
\text { - Higher BPA of the vertebrae } \\
\text { aBMD decreases with age: trend toward } \\
\text { slower decrease in T2DM. }\end{array}$ \\
\hline [51] & Bulló et al. & $\begin{array}{l}251 \\
\text { (110 T2DM; } \\
141 \text { non-DM) }\end{array}$ & $\begin{array}{l}\text { Age: Mean }>65 \text {. } \\
\text { Spanish } \\
50.6 \% \text { women }\end{array}$ & $\begin{array}{l}\text { QUS: BUA, BMD, } \\
\text { QUI, SOS }\end{array}$ & Sex, age, physical activity & $\begin{array}{l}\text { T2DM (or metabolic syndrome): } \\
\text { - Higher BUA, more solid bone structure }\end{array}$ \\
\hline
\end{tabular}




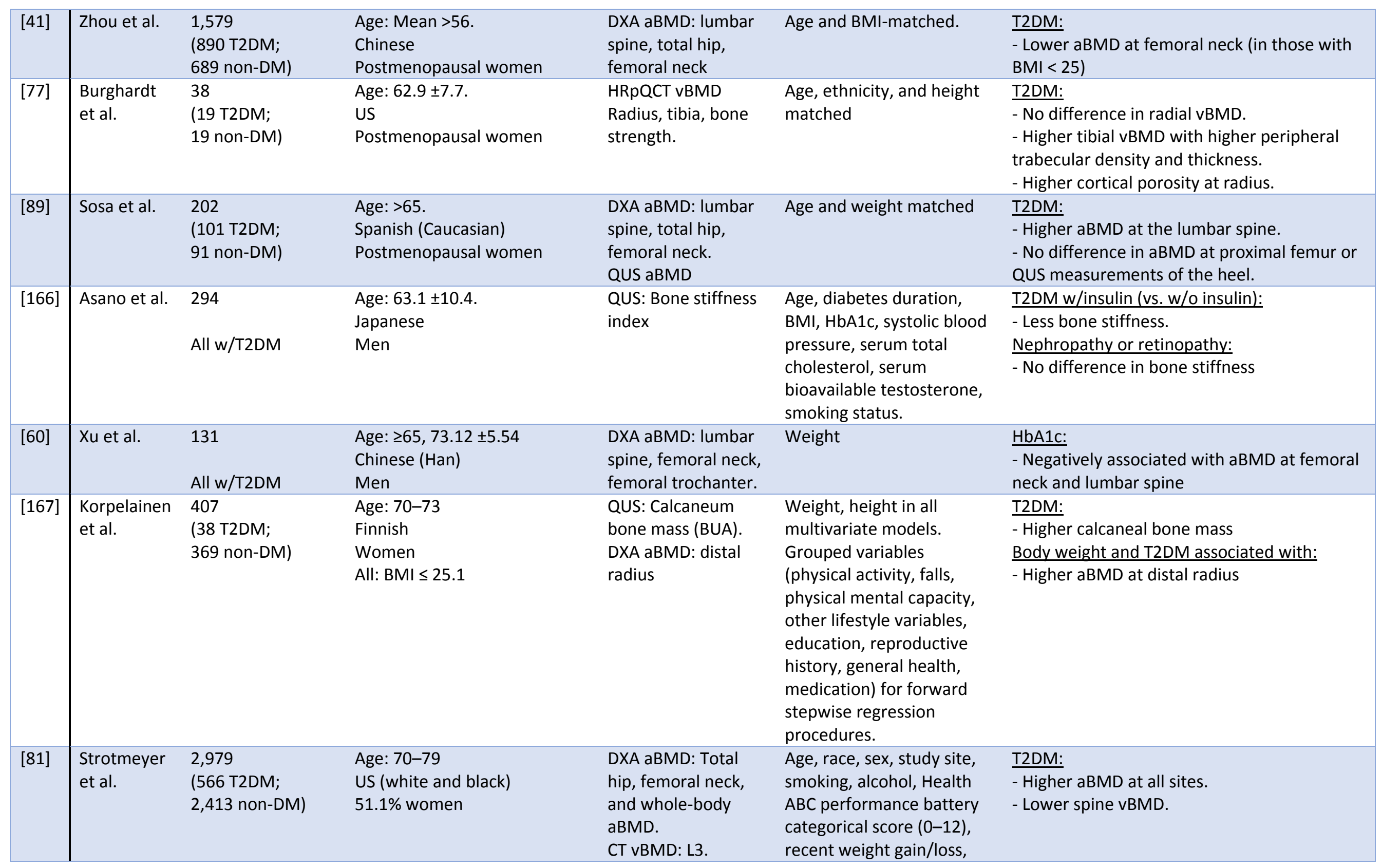




\begin{tabular}{|c|c|c|c|c|c|c|}
\hline & & & & & $\begin{array}{l}\text { osteoporosis, calcium and } \\
\text { vitamin D supplement, } \\
\text { medicationsa. }\end{array}$ & \\
\hline [168] & $\begin{array}{l}\text { Horiuchi et } \\
\text { al. }\end{array}$ & $\begin{array}{l}85 \\
\text { All w/T2DM }\end{array}$ & $\begin{array}{l}\text { Age: Mean > } 69 \\
\text { Japanese } \\
\text { Women. }\end{array}$ & $\begin{array}{l}\text { DXA aBMD: lumbar } \\
\text { spine, T-score }\end{array}$ & Unadjusted & $\frac{\text { T-score }<-2.5 \text { (vs. T-score } \geq-2.5 \text { ): }}{\text { - No difference in HbA1c }}$ \\
\hline$[22]$ & $\begin{array}{l}\text { Dennison et } \\
\text { al. }\end{array}$ & $\begin{array}{l}909 \\
\text { (65 T2DM; } \\
844 \text { non-DM) }\end{array}$ & $\begin{array}{l}\text { Age: } 59-71 \text {, mean }>64 \\
\text { Hertfordshire (UK) } \\
48.8 \% \text { women } \\
\text { None w/DM at inclusion }\end{array}$ & $\begin{array}{l}\text { DXA aBMD: lumbar } \\
\text { spine, total hip, } \\
\text { femoral neck. }\end{array}$ & $\begin{array}{l}\text { Body weight, age, smoking, } \\
\text { alcohol, social class, } \\
\text { activity level, replacement } \\
\text { therapy and menopausal } \\
\text { status (in women) }\end{array}$ & $\begin{array}{l}\text { Newly diagnosed T2DM (vs. controls): } \\
\text { - Only lumbar spine aBMD in men was higher } \\
\text { Insulin resistance or glucose levels: } \\
\text { - No aBMD difference }\end{array}$ \\
\hline [61] & Sert et al. & $\begin{array}{l}539 \\
\text { (277 T2DM; } \\
262 \text { non-DM) }\end{array}$ & $\begin{array}{l}\text { Age } 30-60 \text {, subgroup } 51-60 \\
\text { Turkish } \\
65.3 \% \text { women }\end{array}$ & $\begin{array}{l}\text { DXA aBMD: lumbar } \\
\text { spine, total hip, } \\
\text { femoral neck. }\end{array}$ & $\begin{array}{l}\text { Age- and sex-matched } \\
\text { controls }+ \\
\text { age sub groups }\end{array}$ & $\begin{array}{l}\text { T2DM: } \\
\text { - Higher femoral neck aBMD (in men and } \\
\text { women aged 51-60) } \\
\text { - Higher total femoral aBMD (in men aged 51- } \\
60 \text { ) } \\
\text { - Lower spine aBMD (in men aged 51-60) }\end{array}$ \\
\hline [53] & $\begin{array}{l}\text { Tuominen } \\
\text { et al. }\end{array}$ & $\begin{array}{l}622 \\
\text { (68 T2DM; } \\
56 \text { T1DM; } \\
498 \text { non-DM) }\end{array}$ & $\begin{array}{l}\text { Age: Mean }>60 \\
\text { Finnish } \\
51.3 \% \text { women }\end{array}$ & $\begin{array}{l}\text { DXA aBMD: femoral } \\
\text { neck, trochanter. }\end{array}$ & $\begin{array}{l}\text { Age, BMI, duration of } \\
\text { diabetes, duration of } \\
\text { insulin therapy, physical } \\
\text { activity, calcium intake, } \\
\text { use of estrogens. }\end{array}$ & $\begin{array}{l}\text { T2DM: } \\
\text { - No difference in femoral neck or trochanter } \\
\text { aBMD } \\
\text { T1DM: } \\
\text { - Lower femoral neck aBMD than T2DM } \\
\text { - Lower femoral neck and trochanter aBMD } \\
\text { than controls }\end{array}$ \\
\hline [138] & $\begin{array}{l}\text { Barrett- } \\
\text { Connor et } \\
\text { al. }\end{array}$ & $\begin{array}{l}970 \\
\text { None w/DM at } \\
\text { inclusion }\end{array}$ & $\begin{array}{l}\text { Age: } 67 \pm 8.9 \\
\text { US (Caucasian) } \\
57.6 \% \text { women }\end{array}$ & $\begin{array}{l}\text { DXA aBMD: lumbar } \\
\text { spine, hip, radius. }\end{array}$ & $\begin{array}{l}\text { Age, BMI, waist-to-hip } \\
\text { ratio, family history of } \\
\text { diabetes, exercise, thiazide } \\
\text { use, smoking, estrogen use }\end{array}$ & $\begin{array}{l}\text { T2DM: } \\
\text { - Higher aBMD at lumbar spine, femoral neck } \\
\text { and radius (only in women) }\end{array}$ \\
\hline
\end{tabular}


OBM Geriatrics 2020; 4(2), doi:10.21926/obm.geriatr.2002123

\begin{tabular}{|c|c|c|c|c|c|c|}
\hline & & & & & $\begin{array}{l}\text { and age at menopause } \\
\text { (women). }\end{array}$ & \\
\hline [99] & $\begin{array}{l}\text { van Daele } \\
\text { et al. }\end{array}$ & $\begin{array}{l}\text { 5,931 } \\
\text { (578 T2DM; } \\
\text { 5,353 non-DM) }\end{array}$ & $\begin{array}{l}\text { Age: Mean }>67 \\
\text { Dutch } \\
58.2 \% \text { women }\end{array}$ & $\begin{array}{l}\text { DXA aBMD: lumbar } \\
\text { spine, proximal } \\
\text { femur. }\end{array}$ & $\begin{array}{l}\text { Sex, age, BMI, waist-to-hip } \\
\text { ratio, current use of } \\
\text { thiazides, loop diuretics, } \\
\text { and estrogens, smoking, s- } \\
\text { creatinine, impairment in } \\
\text { activities of daily living. }\end{array}$ & $\begin{array}{l}\text { T2DM: } \\
\text { - Higher aBMD at proximal femur and lumbar } \\
\text { spine } \\
\text { High fasting insulin: } \\
\text { - Higher aBMD at radius, spine and hip in } \\
\text { women } \\
\text { - Higher aBMD at hip in men }\end{array}$ \\
\hline [141] & $\begin{array}{l}\text { Rishaug et } \\
\text { al. }\end{array}$ & $\begin{array}{l}72 \\
\text { (36 T2DM; } \\
36 \text { non-DM) }\end{array}$ & $\begin{array}{l}\text { Age: Mean }>60 \\
\text { Norwegian } \\
41.7 \% \text { (postmenopausal) } \\
\text { women }\end{array}$ & $\begin{array}{l}\text { DXA aBMD: lumbar } \\
\text { spine, femoral neck, } \\
\text { whole body. } \\
\text { BMC } \\
\text { QUS (BUA, SOS) }\end{array}$ & $\begin{array}{l}\text { Sex- and age-matched } \\
\text { control. }\end{array}$ & $\begin{array}{l}\text { T2DM: } \\
\text { - No difference in aBMD, BMC, SOS or BUA in } \\
\text { women } \\
\text { - Higher whole body aBMD in men } \\
\text { Insulin levels: } \\
\text { - Positive correlation to femoral neck aBMD in } \\
\text { men }\end{array}$ \\
\hline [169] & Bauer et al. & $\begin{array}{l}9,704 \\
(6 \% \approx 522 \text { T2DM; } \\
95 \% \approx 9,122 \text { non- } \\
\text { DM) }\end{array}$ & $\begin{array}{l}\text { Age: Mean } 71.1 \\
\text { US (non-black) } \\
\text { Women }\end{array}$ & $\begin{array}{l}\text { Single photon } \\
\text { absorptiometry } \\
\text { BMD: distal radius, } \\
\text { mid-radius, } \\
\text { calcaneus. }\end{array}$ & $\begin{array}{l}\text { Age, multivariate } \\
\text { regression } \underline{\text { a }}\end{array}$ & $\begin{array}{l}\frac{\text { T2DM: }}{\text { - Higher aBMD at distal radius }} \\
\text { Highe }\end{array}$ \\
\hline [139] & $\begin{array}{l}\text { Barrett- } \\
\text { Connor et } \\
\text { al. }\end{array}$ & $\begin{array}{l}627 \\
\text { (80 T2DM; } \\
166 \text { IGM; } \\
381 \text { non-DM) }\end{array}$ & $\begin{array}{l}\text { Age: Mean } 72 \\
\text { US } \\
70 \% \text { women }\end{array}$ & $\begin{array}{l}\text { DXA aBMD: radius, } \\
\text { femoral neck, } \\
\text { lumbar spine. }\end{array}$ & $\begin{array}{l}\text { Age, BMI, alcohol, } \\
\text { smoking, exercise, diuretic } \\
\text { use, estrogen }\end{array}$ & $\begin{array}{l}\text { T2DM: } \\
\text { - Higher aBMD at all sites in women } \\
\text { - No difference in men. } \\
\text { Hyperglycemia: } \\
\text { - Higher aBMD at all sites in women } \\
\text { - No difference in men } \\
\text { aBMD increase with post-challenge glucose in } \\
\text { female controls }\end{array}$ \\
\hline [57] & $\begin{array}{l}\text { Akeroyd et } \\
\text { al. }\end{array}$ & $\begin{array}{l}1,137 \\
\text { (142 T2DM; } \\
995 \text { non-DM) }\end{array}$ & $\begin{array}{l}\text { Age: Mean } 56.6 \text { (T2DM) } \\
\text { US (Black, White, Hispanic) } \\
\text { Men }\end{array}$ & $\begin{array}{l}\text { DXA aBMD: lumbar } \\
\text { spine, femoral neck. } \\
\text { Single photon } \\
\text { absorptiometry } \\
\text { BMD: radius. }\end{array}$ & $\begin{array}{l}\text { Age, race/ethnicity, BMI, } \\
\text { physical activity }\end{array}$ & $\begin{array}{l}\text { T2DM: } \\
\text { - No difference in aBMD. }\end{array}$ \\
\hline
\end{tabular}




\begin{tabular}{|c|c|c|c|c|c|c|}
\hline & & & women & TBS & osteoporosis treatment. & \\
\hline [62] & $\begin{array}{l}\text { Yamamoto } \\
\text { et al. }\end{array}$ & $\begin{array}{l}996 \\
\text { (298 T2DM; } \\
698 \text { non-DM) }\end{array}$ & $\begin{array}{l}\text { Age: } 46-89 \text { (means 65/67) } \\
\text { Japanese } \\
76.2 \% \text { (postmenopausal) } \\
\text { women }\end{array}$ & $\begin{array}{l}\text { DXA aBMD: lumbar } \\
\text { spine, femoral neck. } \\
\text { Single photon } \\
\text { absorptiometry } \\
\text { BMD: radius. }\end{array}$ & Unadjusted & $\begin{array}{l}\text { T2DM: } \\
\text { - Higher aBMD at all sites }\end{array}$ \\
\hline [143] & $\begin{array}{l}\text { Patsch et } \\
\text { al. }\end{array}$ & $\begin{array}{l}85 \\
\text { (43 T2DM; } \\
42 \text { non-DM) }\end{array}$ & $\begin{array}{l}\text { Age: } 57 \pm 11.4 / 58 \pm 4.29 \\
\text { Austrian } \\
30.7 \% \text { women }\end{array}$ & HRpQCT radius/tibia & Sex & $\begin{array}{l}\text { T2DM: } \\
\text { - Higher trabecular number and cortical } \\
\text { thickness } \\
\text { - Trend toward higher cortical BMD } \\
\text { Men had higher radial trabecular BMD and } \\
\text { number and cortical thickness }\end{array}$ \\
\hline [34] & $\begin{array}{l}\text { Ardawi et } \\
\text { al. }\end{array}$ & $\begin{array}{l}964 \\
\text { (482 T2DM; } \\
482 \text { non-DM) }\end{array}$ & $\begin{array}{l}\text { Age: Mean } 59.60 \pm 7.90 \\
\text { Saudi Arabians } \\
\text { Postmenopausal women }\end{array}$ & $\begin{array}{l}\text { DXA aBMD: Lumbar } \\
\text { spine, femoral neck }\end{array}$ & Unadjusted & $\begin{array}{l}\text { T2DM: } \\
\text { - Higher lumbar spine and femoral neck BMD }\end{array}$ \\
\hline [33] & $\begin{array}{l}\text { García- } \\
\text { Martín et } \\
\text { al. }\end{array}$ & $\begin{array}{l}124 \\
\text { (74 T2DM; } \\
50 \text { non-DM) }\end{array}$ & $\begin{array}{l}\text { Age: } 57.7 \pm 6.5 \\
\text { Spanish } \\
52 \% \text { women }\end{array}$ & $\begin{array}{l}\text { DXA BMD and T- } \\
\text { scores: lumbar } \\
\text { spine, femoral neck, } \\
\text { total hip }\end{array}$ & Unadjusted & $\begin{array}{l}\text { T2DM: } \\
\text { - No differences in BMD at any site }\end{array}$ \\
\hline
\end{tabular}

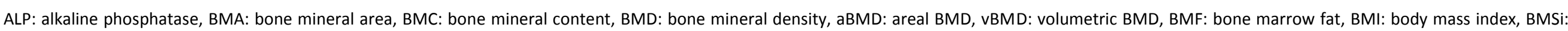

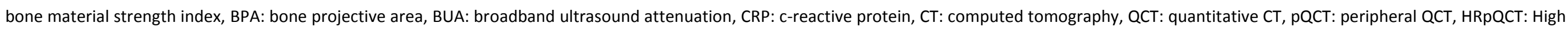

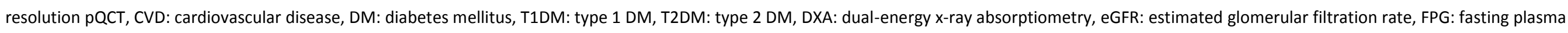

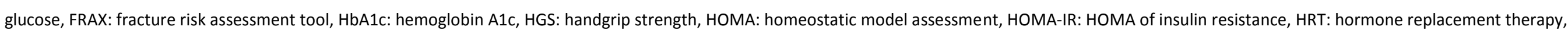

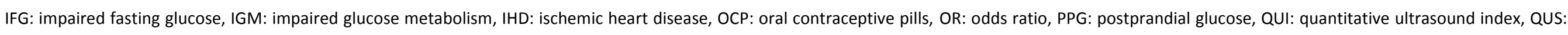

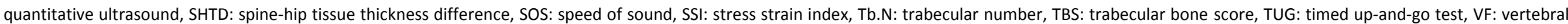
fracture, w/: with, w/o: without 
Table 3 Overview of findings related to fracture risk in type 2 diabetes mellitus.

\begin{tabular}{|c|c|c|c|c|c|c|}
\hline Ref. & Author & $\begin{array}{l}\text { 1) } n=; \\
\text { 2) Follow-up }\end{array}$ & Population & Adjusted for & $\begin{array}{l}\text { Primary (positive) findings: } \\
\text { Effect size }[95 \% \mathrm{Cl}]\end{array}$ & Full conclusion \\
\hline \multicolumn{7}{|c|}{ Meta-analyses } \\
\hline \multicolumn{7}{|c|}{ Randomized Controlled Trials } \\
\hline [52] & $\begin{array}{l}\text { Schwartz et } \\
\text { al. }\end{array}$ & $\begin{array}{l}7,287 \\
(3,655 \\
\text { intensive } \\
\text { glycemia; } \\
3,632 \text { standard } \\
\text { glycemia) } \\
\text { Mean } 3.8 \\
\underline{( \pm 1.3) \text { yrs FU }}\end{array}$ & $\begin{array}{l}\text { Age: mean } 62 \\
\text { US and Canada } \\
34.6 \% \text { women } \\
\text { All: T2DM and } \\
\text { history of, } \\
\text { subclinical evidence } \\
\text { of, or significant risk } \\
\underline{\text { factors for CVD }}\end{array}$ & $\begin{array}{l}\text { Assignment to blood pressure } \\
\text { or lipid trial, randomization to } \\
\text { blood pressure or lipid } \\
\text { intervention, baseline history } \\
\text { of CVD }\end{array}$ & & $\begin{array}{l}\text { Intensive glycemic control vs. } \\
\text { Standard glycemic control: } \\
\text { - No effect on fracture risk } \\
\text { (nonspine, hip, ankle, foot, } \\
\text { proximal humerus, distal forearm) }\end{array}$ \\
\hline \multicolumn{7}{|c|}{ Prospective Cohort Studies } \\
\hline$[100]$ & Tebé et al. & $\begin{array}{l}\text { 126,035 } \\
\text { (44,802 T2DM; } \\
81,233 \text { non- } \\
\text { DM) } \\
\underline{8 y \text { FU }}\end{array}$ & $\begin{array}{l}\text { Age: } 65-80, \text { mean } \\
72 \\
\text { Spanish } \\
53 \% \text { women }\end{array}$ & $\begin{array}{l}\text { Age, corticoid use, calcium + } \\
\text { Vit-D, antiosteoporotic use. } \\
\text { Previous: IHD, CVD, major } \\
\text { fracture, nephropathy }\end{array}$ & $\begin{array}{l}\text { Hip fracture in T2DM vs. Non-DM: } \\
\text { HR } 1.24 \text { (men) } \\
\text { HR } 1.48 \text { (women) }\end{array}$ & $\begin{array}{l}\text { T2DM associated with: } \\
\text { - Increased risk of hip fracture. } \\
\text { - Increased post-hip fracture } \\
\text { mortality. }\end{array}$ \\
\hline$[90]$ & Iki et al. & $\begin{array}{l}\text { 1,951 } \\
\text { (200 T2DM; } \\
\text { 1,751 non-DM) }\end{array}$ & $\begin{array}{l}\text { Age: } \geq 65 \text {, mean } 73 \\
\text { Japanese } \\
\text { Men }\end{array}$ & $\begin{array}{l}\text { Spine- and hip aBMD, } \\
\text { triglycerides, insulin use (ever), } \\
\text { current antidiabetic }\end{array}$ & $\begin{array}{l}\text { Every } 1 \text { SD increase in HbA1c: HR for OPF } \\
\text { of } \mathbf{1 . 3 8} \text { [1.10-1.73] and a HR for MOPF of }\end{array}$ & $\begin{array}{l}\text { HbA1c and FPG linearly correlated } \\
\underline{\text { with: }} \\
\text { - Risk of osteoporotic fractures }\end{array}$ \\
\hline
\end{tabular}




\begin{tabular}{|c|c|c|c|c|c|c|}
\hline & & $\begin{array}{l}\frac{\text { Median FU }}{54.3 \mathrm{mo}} \\
\frac{8480 \text { person- }}{\underline{\mathrm{yrs}}}\end{array}$ & & medications, comorbiditiesa & $\begin{array}{l}1.48[\mathbf{1 . 1 6}-\mathbf{1 . 8 8}] \\
\text { Every } 1 \text { SD increase in FPG: HR for OPF of } \\
1.39[1.15-1.69] \text { and a HR for MOPF of } \\
1.45[1.18-1.79]\end{array}$ & $\begin{array}{l}\text { (OPF) } \\
\text { - Risk of major osteoporotic } \\
\text { fracture (MOPF) } \\
\text { No effect of diabetes status, } \\
\text { HOMA-IR or HOMA-ß. }\end{array}$ \\
\hline$[101]$ & Tebé et al. & $\begin{array}{l}158,984 \\
\text { (55,891 T2DM, } \\
103,093 \text { non- } \\
\text { DM) } \\
\text { Median 6.4/8.0 } \\
\text { yrs FU } \\
\frac{\text { T2DM/non- }}{\text { DM) }} \\
\frac{1,053,768}{\text { person-yrs }}\end{array}$ & $\begin{array}{l}\text { Age: mean } 75 \\
\text { Catalonia, Spain } \\
56.3 \% \text { women }\end{array}$ & $\begin{array}{l}\text { Comorbidities }{ }^{\mathrm{a}} \text {, medications }{ }^{\mathrm{a}} \text {. } \\
\text { Age- and sex-matched }\end{array}$ & $\begin{array}{l}\text { HR } 1.31 \text { [1.23-1.40] for hip fracture } \\
\text { Subhazard ratio (SHR) } 1.15 \text { [1.09-1.21] } \\
\text { (corrected for death) }\end{array}$ & $\begin{array}{l}\text { T2DM increases risk of hip fracture } \\
\text { even after correcting for death as a } \\
\text { competing event. }\end{array}$ \\
\hline [75] & Napoli et al. & $\begin{array}{l}\text { 5,554 } \\
\text { (875 T2DM; } \\
4,679 \text { non-DM) } \\
\text { Mean } 4.6 \text { yr FU } \\
\end{array}$ & $\begin{array}{l}\text { Age: } \geq 65 \text {, mean } 73 \\
\text { US Citizens } \\
\text { Men }\end{array}$ & $\begin{array}{l}\text { Age, race, clinical site, } \mathrm{BMI} \text {, } \\
\text { aBMD/vBMD }\end{array}$ & & $\begin{array}{l}\text { T2DM not associated with: } \\
\text { - Prevalent vertebral fracture } \\
\text { - Incident vertebral fracture }\end{array}$ \\
\hline [124] & $\begin{array}{l}\text { Wallander } \\
\text { et al. }\end{array}$ & $\begin{array}{l}428,305 \\
\text { (79,159 T2DM; } \\
\text { 5,543 T1DM; } \\
343,603 \text { non- } \\
\text { DM) } \\
\frac{\text { Median } 1.3 \text { yrs }}{\underline{\text { FU }}} \\
\frac{670,000 \text { person }}{y r s}\end{array}$ & $\begin{array}{l}\text { Age: mean } 79-81 \\
\text { Swedish } \\
57.6 \% \text { women }\end{array}$ & $\begin{array}{l}\text { Age, sex, height, weight, } \\
\text { (insulin use) }\end{array}$ & $\begin{array}{l}\text { Compared to Non-DM: } \\
\text { T2DM total: HR for hip fracture } 1.10 \\
\text { [1.05-1.15]. No correlation after adjusting } \\
\text { for insulin use. } \\
\text { T2DM w/oral medication or insulin } \\
\text { (women vs. men): HR 1.26 [1.04- } \\
\text { 1.53] and HR } 1.42 \text { [1.19-1.68], } \\
\text { respectively. } \\
\text { T2DM w/o medication: HR for hip fracture } \\
\text { in men } 0.78 \text { [0.64-0.94]. Not reduced in } \\
\text { women. }\end{array}$ & $\begin{array}{l}\text { T2DM w/o medication (vs. non- } \\
\text { DM): } \\
\text { - Unaffected overall fracture risk } \\
\text { (men/total), increased in women } \\
\text { - Hip fracture risk reduced in men } \\
\text { but increased in women } \\
\text { T2DM w/oral medication or insulin } \\
\text { (women vs. men): } \\
\text { - Larger increase in risk of hip, any, } \\
\text { MOPF, ankle, upper arm fracture } \\
\text { T2DM w/insulin (vs. non-DM): }\end{array}$ \\
\hline
\end{tabular}




\begin{tabular}{|c|c|c|c|c|c|c|}
\hline & & & & & $\begin{array}{l}\text { Any diabetic complication (DM type I or } \\
\text { II): HR for hip fracture 1.16 [1.06-1.26]. } \\
\text { Not significant after adjusting for insulin } \\
\text { use. } \\
\text { Retinopathy: HR for hip fracture } 1.17 \\
\text { [1.06-1.29]. Not significant after adjusting } \\
\text { for insulin use. }\end{array}$ & $\begin{array}{l}\text { - Increased risk of any fracture, } \\
\text { MOPF, upper arm, hip and ankle } \\
\text { fracture } \\
\text { T2DM total (vs. non-DM): } \\
\text { - Increased hip fracture risk (fully } \\
\text { attenuated by correction for } \\
\text { insulin) } \\
\text { T1DM vs. T2DM: Risk of any, hip } \\
\text { MOPF and ankle fractures higher in } \\
\text { T1DM than in all T2DM medication } \\
\text { subgroups. }\end{array}$ \\
\hline [65] & $\begin{array}{l}\text { Mitama et } \\
\text { al. }\end{array}$ & $\begin{array}{l}\text { 6,556 } \\
\text { (792 T2DM; } \\
649 \text { IGM; } \\
\text { 5,115 non-DM) } \\
\text { Mean FU } 7.4 \\
\text { yrs }\end{array}$ & $\begin{array}{l}\text { Age: mean } 67-68 \\
\text { Japanese } \\
57.5 \% \text { women }\end{array}$ & $\begin{array}{l}\text { age, BMD, eGFR, albumin, } \\
\text { exercise, smoking, alcohol, } \\
\text { family history of fracture, IHD, } \\
\text { CVD }\end{array}$ & $\begin{array}{l}\text { Compared to HbA1c } \leq 5.6 \% \text { : } \\
\text { HbA1c } 5.7 \%-6.4 \%: \text { No increased risk } \\
\text { HbA1c } \geq 6.5 \% \text { (DM): HR for incident } \\
\text { fracture } 1.31[1.02-1.51] \text { in men, not } \\
\text { significant in women. }\end{array}$ & $\begin{array}{l}\text { T2DM associated with: } \\
\text { - Increased risk of hip fracture in } \\
\text { men } \\
\text { - No increased risk in women }\end{array}$ \\
\hline [103] & Kim et al. & $\begin{array}{l}51,330 \\
(17,110 \text { T2DM, } \\
34,220 \text { non- } \\
\text { DM) } \\
7 \text { yrs FU (or } \\
\text { until death) }\end{array}$ & $\begin{array}{l}\text { Age: } \geq 50 \\
\text { Korean } \\
54 \% \text { women }\end{array}$ & $\begin{array}{l}\text { Age, household income, } \\
\text { comorbiditiesa , steroid use, } \\
\text { osteoporosisa }\end{array}$ & $\begin{array}{l}\text { Fracture in diabetics vs. controls: } \\
\text { Hip: HR 1.73 [1.38-2.16] in women, HR } \\
1.84[1.29-2.63] \text { in men }\end{array}$ & $\begin{array}{l}\text { T2DM associated with: } \\
\text { - Increased hip fracture risk } \\
\text { (irrespective of sex) } \\
\text { - No increased risks of non- } \\
\text { vertebral, vertebral or any fracture }\end{array}$ \\
\hline [105] & $\begin{array}{l}\text { Majumdar } \\
\text { et al. }\end{array}$ & $\begin{array}{l}57,938 \\
(8,840 \text { T2DM; } \\
49,098 \text { non- } \\
\text { DM) } \\
\frac{0-17 \text { yrs FU }}{\text { (mean } 7 \text { yrs }])} \\
>420,000\end{array}$ & $\begin{array}{l}\text { Age: mean } 73 \\
\text { Canadian } \\
\text { Women } \\
\text { All: Undergoing } \\
\text { DXA, } \geq 10 \text { years of } \\
\text { health coverage }\end{array}$ & $\begin{array}{l}\text { FRAX-scores: computed with } \\
\text { BMD, BMI, prior fracture. } \\
\text { Comorbiditya , falls, } \\
\text { antiosteoporotic drugs, insulin. }\end{array}$ & $\begin{array}{l}\text { T2DM vs. Non-DM: } \\
\text { Prior fracture: } 16.5 \% \text { vs. } 14.3 \%(p<.0001) \\
\text { MOPF: } 9.2 \% \text { vs. } 8.6 \%(p=0.05) \\
\text { Hip: } 3.2 \% \text { vs. } 2.3 \%(p<0.0001) \\
\text { Vertebral: } 2.3 \% \text { vs. } 1.9 \%(p=0.04) \\
\text { Humerus: } 2.3 \% \text { vs. } 1.7 \%(p<0.0001) \\
\text { Foot: } 2.8 \% \text { vs. } 3.7 \%(p<0.0001)\end{array}$ & $\begin{array}{l}\text { T2DM in women is associated with: } \\
\text { - Increased risk of hip fracture - } \\
\text { regardless of duration. } \\
\text { - Increased risk of MOPF - only if } \\
\text { long disease duration. } \\
\text { - Increased risk of vertebral, } \\
\text { humerus and forearm fracture }\end{array}$ \\
\hline
\end{tabular}




\begin{tabular}{|c|c|c|c|c|c|c|}
\hline & & person-years & & & $\begin{array}{l}\text { Ankle: } 1.7 \% \text { vs. } 1.5 \%(p=0.2) \\
\text { Diabetes duration groups vs. non-DM: } \\
\text { New onset: HR 0.99 [0.86-1.14] for MOPF, } \\
\text { HR } 1.30 \text { [1.01-1.65] for HF } \\
<5 y: \text { HR 1.07 [0.92-1.25] for MOPF, HR } \\
\text { 1.54 [1.19-1.99] for HF } \\
5-10 \text { y: HR 1.10 [0.93-1.29] for MOPF, HR } \\
\text { 1.55 [1.17-2.06] for HF } \\
\text { '> } 10 \text { y': HR 1.47 [1.30-1.66] for MOPF, HR } \\
\text { 1.94 [1.54-2.44] for HF }\end{array}$ & $\begin{array}{l}\text { - Increased prevalence of prior } \\
\text { fracture }\end{array}$ \\
\hline [107] & $\begin{array}{l}\text { Hamilton et } \\
\text { al. }\end{array}$ & $\begin{array}{l}6,450 \\
(1291 \text { T2DM; } \\
5159 \text { non-DM) } \\
\text { Mean } 14.1 \\
\underline{ \pm 5.9) \text { yrs FU }} \\
\underline{90,808 \text { patient- }} \\
\underline{\text { years }}\end{array}$ & $\begin{array}{l}\text { Age: } \geq 55 \\
\text { Australian } \\
51.3 \% \text { women }\end{array}$ & $\begin{array}{l}\text { Age, sex, comorbidities } \\
\text { (Charlson Comorbidity Index } \\
\text { excluding diabetes-specific } \\
\text { chronic complications) }\end{array}$ & $\begin{array}{l}\text { First hip fracture (T2DM vs. non-DM): } \\
\text { Age 75-84: Crude IRR 1.80 [1.26-2.55] } \\
\text { All ages: Crude IRR 1.33 [1.05-1.68] (0= } \\
\text { 0.013) } \\
\text { All ages: csHR } 1.50 \text { [1.19-1.89] ( } p=0.001 \text { ) } \\
\text { All ages: sdHR } 1.21 \text { [0.96-1.52] ( } p=0.11 \text { ) } \\
\text { First hip fracture (predictors): } \\
\text { Diabetes duration (increase of } 1 \text { y): csHR } \\
\text { 1.02 [1.01-1.03], } p<0.001 \\
\text { HbA1c (increase of 1\%): csHR 1.07 [1.02- } \\
\text { 1.13], } p=0.005 \\
1 \text { unit increase in In(urine-A:C-ratio): csHR } \\
\text { 1.1 [1.13-1.26], } p=0.001 \\
\text { Peripheral sensory neuropathy: csHR 1.38 } \\
\text { [1.16-1.64], } p<0.001 \\
\text { All incident hip fractures (T2DM vs. non- } \\
\text { DM): } \\
\text { All ages: Crude IRR 1.28 [1.02-1.59] ( } \mathbf{0}= \\
\mathbf{0 . 0 2 9 )} \\
\text { Age groups (10 yr intervals): IRR between }\end{array}$ & $\begin{array}{l}\text { T2DM associated with: } \\
\text { - Increased risk of first and all } \\
\text { incident hip fractures (HF) - this } \\
\text { effect only found in subgroup with } \\
\text { age } 75-84 . \\
\text { Effect on first incident HF } \\
\text { attenuated after allowing for } \\
\text { competing risk of death } \\
\text { Peripheral sensory neuropathy } \\
\text { (PSN) associated with: } \\
\text { - Increased risk of multiple incident } \\
\text { hip fracture (HF) } \\
\text { First incident HF risk increased by: } \\
\text { - Diabetes duration, HbA1c, } \\
\text { proteinuria, PSN }\end{array}$ \\
\hline
\end{tabular}




\begin{tabular}{|c|c|c|c|c|c|c|}
\hline & & & & & $\begin{array}{l}\text { 1.60-1.87 } \\
\text { Multiple incident HF: } \\
\text { Peripheral sensory neuropathy vs. no } \\
\text { neuropathy: RR } 1.65 \text { [1.07-2.54] }\end{array}$ & \\
\hline [122] & $\begin{array}{l}\text { Conway et } \\
\text { al. }\end{array}$ & $\begin{array}{l}10,572 \\
\text { All w/T2DM } \\
\text { Mean } 3.3 \mathrm{yrs} \\
\underline{\mathrm{FU}}\end{array}$ & $\begin{array}{l}\text { Age: mean } 71 \\
\text { US Citizens } \\
50.8 \% \text { women }\end{array}$ & $\begin{array}{l}\text { Model 1: age, sex, race } \\
\text { Model 2: age, sex, race, } \\
\text { number of BMI-measurements }\end{array}$ & $\begin{array}{l}\text { Compared to HbA1c } 7.0 \%-7.9 \% \text { (HRs of } \\
\text { model 1, model 2): } \\
\text { HbA1c < 6.5\%: HR 0.96 [0.81-1.13], HR } \\
0.97 \text { [0.82-1.14] } \\
\text { HbA1c 6.5\%-6.9\%: HR } 0.77 \text { [0.64-0.93], } \\
\text { HR 0.80 [0.66-0.97] } \\
\text { HbA1c 8.0\%-8.9\%: HR } 1.22 \text { [0.99-1.50], } \\
\text { HR 1.13 [0.92-1.40] } \\
\text { HbA1c } \geq 9 \%: \text { HR 1.55 [1.21-2.00], HR } 1.19 \\
\text { [0.93-1.54] }\end{array}$ & $\begin{array}{l}\text { Risk of any fracture: } \\
\text { - Lower in HbA1c } 6.5-6.9 \% \text {. } \\
\text { - Highest in } \mathrm{HbA} 1 \mathrm{c} \geq 9 \% \text {. } \\
\text { Risk attenuated in adjustment } \\
\text { model } 2\end{array}$ \\
\hline [127] & $\begin{array}{l}\text { Martinez- } \\
\text { Laguna et al. }\end{array}$ & $\begin{array}{l}\text { 171,931 } \\
\text { (58,483 T2DM; } \\
113,448 \text { non- } \\
\text { DM) } \\
\text { Median } 2.63 \\
\text { yrs FU }\end{array}$ & $\begin{array}{l}\text { Age: mean } 62 \\
\text { Spanish } \\
43.5 \% \text { women }\end{array}$ & $\begin{array}{l}\text { All age- and sex-matched. } \\
\text { *BMI, previous fracture, oral } \\
\text { corticoids. } \\
{ }^{* *} \text { Prevalent CVD, IHD, CKD } \\
\text { and falls history. }\end{array}$ & $\begin{array}{l}\text { Subhazard ratio (SHR) in T2DM vs. Non- } \\
\text { DM: } \\
\text { *Partially adjusted risk of HF: SHR } 1.20 \\
\text { [1.06-1.35] } \\
\text { **Fully adjusted risk of HF: SHR } 1.10 \\
\text { [0.98-1.24] } \\
\geq 1 \text { osteoporotic fracture: SHR } 0.97 \text { [0.92- } \\
1.02] \\
\text { Major osteoporotic fracture: SHR } 0.95 \\
\text { [0.89-1.01] }\end{array}$ & $\begin{array}{l}\text { T2DM associated with: } \\
\text { - 20\% increased risk of fracture; } \\
\text { non-significant after adjustment } \\
\text { for comorbidities and falls. } \\
\text { Significant interaction between } \\
\text { T2DM and BMI, CKD and IHD on } \\
\text { hip fracture risk. }\end{array}$ \\
\hline [108] & Lee et al. & $\begin{array}{l}\text { 70,829 } \\
\text { (4,805 T2DM; } \\
66,024 \text { non- } \\
\text { DM) } \\
\text { "EPESE study: } \\
\text { Mean 6.5/8.1 }\end{array}$ & $\begin{array}{l}\text { Age: means } 73-74, \\
61-63 \\
\text { USA } \\
\text { Postmenopausal } \\
\text { women }\end{array}$ & $\begin{array}{l}\text { *Age, race, BMI } \\
{ }^{* *} \text { Age, race, BMI, functional } \\
\text { impairments, comorbidityạ, } \\
\text { alcohol use, tobacco use, } \\
\text { vision impairment, } \\
\text { medicationsa. }\end{array}$ & $\begin{array}{l}\text { Hazard ratio in EPESE and WHI study: } \\
\text { *Any fracture (AF): HR 1.36 [1.08-1.72] } \\
\text { and HR } 1.29 \text { [1.19-1.39] } \\
\text { **AF fully adjusted: HR } 1.22 \text { [0.96-1.56] } \\
\text { and HR 1.20 [1.11-1.30] } \\
\text { *Hip fracture (HF): HR 1.27 [0.80- }\end{array}$ & $\begin{array}{l}\text { T2DM associated with: } \\
\text { - 29-36\% increased risk of any } \\
\text { clinical fracture; attenuated in full } \\
\text { model adjustment in one study } \\
\text { (EPESE). } \\
\text { - Hip fracture risk only in WHI }\end{array}$ \\
\hline
\end{tabular}




\begin{tabular}{|c|c|c|c|c|c|c|}
\hline & & $\begin{array}{l}\text { yrs FU } \\
\text { (EPESE/WHI } \\
\text { studies) }\end{array}$ & & & $\begin{array}{l}\text { 2.20] and HR } 1.45 \text { [1.08-1.94] } \\
\text { **HF fully adjusted: HR } 1.08 \text { [0.66- } \\
\text { 1.76] and HR } 1.28 \text { [0.95-1.73] } \\
\text { *NHNVF: HR } 1.23 \text { [0.97-1.56] and HR } 1.28 \\
\text { [1.18-1.39] } \\
\text { **NHNVF fully adjusted: HR } 1.13 \text { [0.87- } \\
\text { 1.46] and HR } 1.20 \text { [1.10-1.31] }\end{array}$ & $\begin{array}{l}\text { study (by } 45 \%) ; \text { attenuated in full } \\
\text { model adjustment } \\
\text { - Risk of non-hip, non-vertebral } \\
\text { fracture in one study by } 28 \% \\
\text { (WHI), still significant after full } \\
\text { model adjustment }\end{array}$ \\
\hline [109] & Reyes et al. & $\begin{array}{l}\text { 186,171 } \\
\text { (36,865 T2DM; } \\
\text { 149,306 non- } \\
\text { DM) } \\
\text { Median } 2.99 \\
\text { yrs FU }\end{array}$ & $\begin{array}{l}\text { Age: mean } 76 / 84 \\
\text { Catalonia, Spain } \\
\text { Men }\end{array}$ & $\begin{array}{l}\text { Age, BMI, smoking, alcohol } \\
\text { consumption, oral } \\
\text { corticosteroids, comorbidities } \underline{\text { a }}\end{array}$ & $\begin{array}{l}\text { Relative risk for hip fracture: } \\
\text { T2DM (vs. Non-DM): RR } 1.45 \text { [1.25-1.69], } \\
\mathbf{p}<0.001 \\
\text { T2DM w/complications: RR } 1.89 \text { [1.15- } \\
\text { 3.21], p < } 0.012\end{array}$ & $\begin{array}{l}\text { Hip fracture associated with: } \\
\text { - T2DM. } \\
\text { - Diabetic complications. }\end{array}$ \\
\hline [110] & $\begin{array}{l}\text { Hothersall } \\
\text { et al. }\end{array}$ & $\begin{array}{l}3,840,841 \\
(180,841 \\
\text { T2DM; } \\
3,660,000 \text { non- } \\
\text { DM) } \\
\underline{461,120} \\
\text { person-yrs } \\
\underline{\underline{(T 2 D M)}} \\
\underline{10,980,599} \\
\underline{\text { person-yrs }} \\
\underline{\text { (non-DM) }}\end{array}$ & $\begin{array}{l}\text { Age: } 50-84 \\
\text { Scottish } \\
\text { Unspecified sex } \\
\text { fractions }\end{array}$ & $\begin{array}{l}\text { Age, calendar year, SIMD } \\
\text { (Scottish Index of Multiple } \\
\text { Deprivation), }\end{array}$ & $\begin{array}{l}\text { Incident Rate Ratio (IRR) of T2DM vs. Non- } \\
\text { DM (by age and sex): } \\
\text { Men 60-69: IRR 0.87 [0.76-1.00], } p= \\
0.046 \\
\text { Men Total: IRR } 0.97 \text { [0.92-1.02], } p=0.234 \\
\text { Women 50-59: IRR 1.21 [1.04-1.41], } p= \\
\mathbf{0 . 0 1 3} \\
\text { Women 60-69: IRR 1.14 [1.08-1.21], } p< \\
\mathbf{0 . 0 0 1} \\
\text { Women 70-79: IRR 1.06 [1.01-1.12], } p= \\
\mathbf{0 . 0 3 2} \\
\text { Women Total: IRR 1.05 [1.01-1.10], p = } \\
\mathbf{0 . 0 1 3} \\
\text { IRR for diabetes duration > seven years vs. } \\
\text { non-DM: } \\
\text { Men: IRR 1.25 [1.08-1.45] } \\
\text { Women: IRR 1.55 [1.38-1.75] }\end{array}$ & $\begin{array}{l}\text { T2DM associated with increased } \\
\text { risk of hip fracture: } \\
\text { - In females (total) and in all age- } \\
\text { subgroups except } 80-84 . \\
\text { - In all diabetics with long disease } \\
\text { duration (> seven years) } \\
\text { T2DM associated with reduced risk } \\
\text { of hip fracture: } \\
\text { - In } 60-69 \text { year old males (not in } \\
\text { full male cohort) }\end{array}$ \\
\hline
\end{tabular}




\begin{tabular}{|c|c|c|c|c|c|c|}
\hline [128] & $\begin{array}{l}\text { Schneider et } \\
\text { al. }\end{array}$ & $\begin{array}{l}\text { 15,140 (1,195 } \\
\text { T2DM; } \\
605 \\
\text { undiagnosed } \\
\text { DM; } \\
13,340 \text { non- } \\
\text { DM) } \\
\underline{\text { Median } 20 \text { yrs }} \\
\underline{\text { FU }}\end{array}$ & $\begin{array}{l}\text { Age: mean } 55 \\
\text { USA } \\
55.4 \% \text { women }\end{array}$ & $\begin{array}{l}\text { Age, sex, race/study center, } \\
\text { BMI, sports activity tertile, } \\
\text { alcohol/smoking, medicationsa }\end{array}$ & $\begin{array}{l}\text { Hazard ratio (HR) for fracture according to } \\
\text { diabetes status at baseline (compared to } \\
\text { non-DM): } \\
\text { Undiagnosed diabetics ( } \mathrm{n}=547 \text { ): HR } 1.12 \\
\text { [0.82-1.53] } \\
\text { Diagnosed diabetics: HR 1.74 [1.42-2.14] } \\
\text { HR for fracture by HbA1c: } \\
\text { HbA1c } \geq 8.0 \% \text { vs. < 8.0\% (T2DM): HR } 1.63 \\
\text { [1.09-2.44] } \\
\text { Undiagnosed diabetics vs. HbA1c <5.7\% } \\
\text { (non-DM): HR } 1.05[0.72-1.54]\end{array}$ & $\begin{array}{l}\text { Higher risk of any fracture } \\
\text { associated with: } \\
\text { - T2DM (only if present at baseline) } \\
\text { - Higher HbA1c }\end{array}$ \\
\hline [112] & $\begin{array}{l}\text { Hippisley- } \\
\text { Cox et al. }\end{array}$ & $\begin{array}{l}3,142,673 \\
(88,540 \text { T2DM; } \\
3,054,133 \text { non- } \\
\text { DM) } \\
\underline{23,608,337} \\
\text { person-yrs }\end{array}$ & $\begin{array}{l}\text { Age: } 50+ \\
\text { UKGB } \\
50.6 \% \text { women }\end{array}$ & $\begin{array}{l}\text { Age, BMI, ethnic origin, alcohol } \\
\text { intake, smoking status, } \\
\text { medical or social factors } \\
\text { (comorbidities, medications) }\end{array}$ & $\begin{array}{l}\text { HR for fracture in women (T2DM vs. Non- } \\
\text { DM): } \\
\text { Osteoporotic fracture: HR 1.27 [1.21- } \\
\text { 1.34] } \\
\text { Hip fracture: HR 1.57 [1.45-1.69] } \\
\text { HR for fracture in men (T2DM vs. Non- } \\
\text { DM): } \\
\text { Osteoporotic fracture: HR 1.25 [1.15- } \\
\text { 1.36] } \\
\text { Hip fracture: HR 1.33 [1.19-1.49] }\end{array}$ & $\begin{array}{l}\text { T2DM associated with (in both } \\
\text { sexes individually): } \\
\text { Higher risk of hip fracture } \\
\text { Higher risk of any osteoporotic } \\
\text { fracture }\end{array}$ \\
\hline [113] & $\begin{array}{l}\text { Schwartz et } \\
\text { al. }\end{array}$ & $\begin{array}{l}\text { 16,885 } \\
\text { (1,969 T2DM; } \\
\text { 14,916 non- } \\
\text { DM) } \\
\text { [3 prospective } \\
\text { studies] } \\
\text {-SOF study: } 2 \\
\text { yrs FU }\end{array}$ & $\begin{array}{l}\text { Age: means } 73 \\
\text { US } \\
56 \% \text { women }\end{array}$ & Unadjusted & $\begin{array}{l}\text { Rate of fracture per } 1000 \text { person-years } \\
\text { (T2DM vs. Non-DM): } \\
\text { Hip, Study } 1 \text { (Women): } 13.4 \pm 1.7 \text { vs. } \\
11.1 \pm 0.3, p<0.0001 \\
\text { Hip, Study } 2 \text { (Men): } 3.0 \pm 0.7 \text { vs. } 3.3 \pm 0.3, p \\
<0.0001 \\
\text { Hip, Study } 3 \text { (Women): } 6.7 \pm 2.1 \text { vs. } \\
5.3 \pm 0.7, p<0.0001 \\
\text { Hip, Study } 3 \text { (Men): } 4.9 \pm 1.5 \text { vs. } 3.3 \pm 0.6, p\end{array}$ & $\begin{array}{l}\text { T2DM associated with rate of } \\
\text { fracture (hip and any } \\
\text { nonvertebral): } \\
\text { - Increased in women in study } \\
\text { "SOF" ( } n=7,926) \\
\text { - Increased in women in study } \\
\text { "Health ABC" ( } n=1,523) \\
\text { - Increased in men in study "Health } \\
A B C "(n=1,442)\end{array}$ \\
\hline
\end{tabular}




\begin{tabular}{|c|c|c|c|c|c|c|}
\hline & & $\begin{array}{l}\frac{- \text { MrOS: } 9 \text { yrs }}{\underline{\text { FU }}} \\
\underline{\underline{- \text { Health ABC: }}} \\
\underline{10 \text { yrs FU }}\end{array}$ & & & $\begin{array}{l}<0.0001 \\
\text { Nonvertebral, Study } 1 \text { (Women): } 51.0 \pm 3.8 \\
\text { vs. } 42.5 \pm 0.8, p<0.0001 \\
\text { Nonvertebral, Study } 2 \text { (Men): } 15.7 \pm 1.7 \text { vs. } \\
16.5 \pm 0.7, p<0.0001 \\
\text { Nonvertebral, Study } 3 \text { (Women): } 23.3 \pm 4.1 \\
\text { vs. } 19.9 \pm 1.4, p<0.0001 \\
\text { Nonvertebral, Study } 3 \text { (Men): } 12.1 \pm 2.5 \text { vs. } \\
9.8 \pm 1.1, p<0.0001 \\
\text { For any given fracture risk (T2DM vs. Non- } \\
\text { DM): } \\
\text { - Women, higher aBMD T-score: Mean } \\
\text { difference } 0.59 \text { [0.31-0.87] } \\
\text { - Men, higher aBMD T-score: Mean } \\
\text { difference } 0.38 \text { [0.09-0.66] }\end{array}$ & $\begin{array}{l}\text { - Reduced in men in study "MrOS" } \\
\text { ( } n=5,994 \text { ) } \\
\text { T2DM vs. Non-DM, both sexes: } \\
\text { - For a given fracture risk, there } \\
\text { was a higher femoral aBMD T- } \\
\text { score } \\
\text { - Conversely, increased fracture } \\
\text { risk for similar T-scores (see article } \\
\text { Figure 1) }\end{array}$ \\
\hline [115] & $\begin{array}{l}\text { Janghorbani } \\
\text { et al. }\end{array}$ & $\begin{array}{l}\text { 109,691 } \\
(8,348 \text { T2DM; } \\
101,343 \text { non- } \\
\text { DM) } \\
\text { Mean } \\
\frac{20.4 / 20.0}{\text { (T2DM/non- }} \\
\text { DM) yrs FU }\end{array}$ & $\begin{array}{l}\text { Age: mean } 62 \\
\text { US } \\
\text { Women }\end{array}$ & $\begin{array}{l}\text { Age, BMI, physical activity, } \\
\text { menopausal status, estrogen } \\
\text { use, smoking, daily intake of } \\
\text { calcium, vitamin D and protein }\end{array}$ & 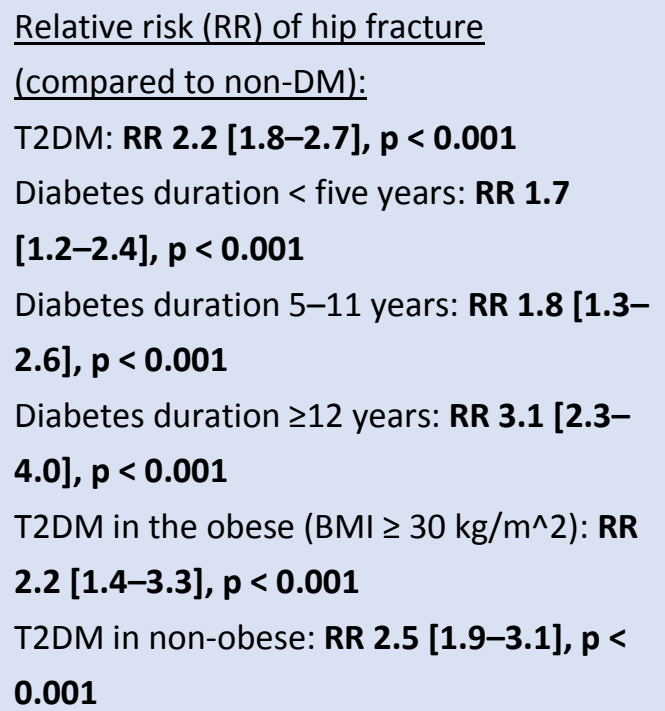 & $\begin{array}{l}\text { T2DM associated with: } \\
\text { - Increased risk of hip fracture } \\
\text { Effect of T2DM is lower in obese } \\
\text { individuals } \\
\text { Diabetes duration ( }<5 \mathrm{yr}, 5-11 \mathrm{yr} \text {, } \\
\geqq 12 \mathrm{yr} \text { ) associated with: } \\
\text { - Increasing risk of hip fracture } \\
\text { with increasing duration }\end{array}$ \\
\hline [31] & Dobnig et al. & $\begin{array}{l}1,664 \\
\text { (583 T2DM; }\end{array}$ & $\begin{array}{l}\text { Age: } \geq 70 \\
\text { Austrian }\end{array}$ & $\begin{array}{l}\text { Age, weight, calcaneal bone } \\
\text { mass }\end{array}$ & $\begin{array}{l}\text { Hazard ratio (HR) for incident hip fracture: } \\
\text { Model 1: T2DM vs. Non-DM: HR } 0.90\end{array}$ & $\begin{array}{l}\text { T2DM associated with: } \\
\text { - Increased risk of hip fracture, only }\end{array}$ \\
\hline
\end{tabular}




\begin{tabular}{|c|c|c|c|c|c|c|}
\hline & & $\begin{array}{l}1,081 \text { non-DM) } \\
\underline{2 \text { yrs FU }}\end{array}$ & Women & & $\begin{array}{l}{[0.60-1.34]} \\
\text { Model 2: T2DM vs. Non-DM: HR } 1.46 \\
{[\mathbf{1 . 2 5 - 1 . 8 1 ] , ~} \mathbf{p}=\mathbf{0 . 0 1}}\end{array}$ & $\begin{array}{l}\text { when adjusted for calcaneal bone } \\
\text { mass. }\end{array}$ \\
\hline [80] & $\begin{array}{l}\text { Strotmeyer } \\
\text { et al. }\end{array}$ & $\begin{array}{l}2,979 \\
(566 \text { T2DM; } \\
177 \text { IFG; } \\
2,236 \text { NGM) } \\
5.4 \pm 1.1 \text { yrs FU } \\
\end{array}$ & $\begin{array}{l}\text { Age: mean } 73-74 \\
\text { US (white and } \\
\text { black) } \\
51.1 \% \text { women }\end{array}$ & $\begin{array}{l}\text { Sex, race, age, site, hip BMD, } \\
\text { lean mass, fat mass, visceral } \\
\text { fat }\end{array}$ & $\begin{array}{l}\text { Relative risk (RR) for any fracture } \\
\text { (compared to NGM): } \\
\text { T2DM: RR 1.64 [1.07-2.51] } \\
\text { Impaired fasting glucose: RR } 1.34 \text { [0.67- } \\
\text { 2.67] }\end{array}$ & $\begin{array}{l}\text { Overall fracture risk associated } \\
\text { with: } \\
\text { - T2DM. } \\
\text { No association with: } \\
\text { - Diabetes duration } \\
\text { - HbA1C }\end{array}$ \\
\hline [32] & $\begin{array}{l}\text { Gerdhem et } \\
\text { al. }\end{array}$ & $\begin{array}{l}1,132 \\
\text { (74 T2DM; } \\
1,058 \text { non-DM) } \\
\text { Mean } 4.6 \text { yrs } \\
\underline{\text { FU }}\end{array}$ & $\begin{array}{l}\text { Age: All } 75 \\
\text { Swedish } \\
\text { Women }\end{array}$ & Unadjusted & & $\begin{array}{l}\text { T2DM not associated with fracture } \\
\text { (any, hip, forearm, vertebral): } \\
\text { - Lifetime risk, risk after the age of } \\
75, \text { age at first fracture } \\
\text { Prevalent fracture not associated } \\
\text { with: } \\
\text { - Diabetes duration }\end{array}$ \\
\hline [123] & $\begin{array}{l}\text { de Liefde et } \\
\text { al. }\end{array}$ & $\begin{array}{l}6,655 \\
\text { (792 T2DM; } \\
5,863 \text { non-DM) } \\
\text { Mean } 6.8 \text { yrs } \\
\underline{\text { FU }}\end{array}$ & $\begin{array}{l}\text { Age: } \geq 55 \text { (mean } 74 \text { ) } \\
\text { Dutch } \\
59.6 \% \text { women }\end{array}$ & $\begin{array}{l}\text { Age, sex, BMI, smoking, s- } \\
\text { creatinine, visual acuity, falling } \\
\text { frequency, lower limb } \\
\text { disability, femoral neck BMD }\end{array}$ & $\begin{array}{l}\text { Fracture in the previous five years (T2DM } \\
\text { vs. Non-DM): } \\
\text { Nonvertebral fracture: } 13,5 \% \text { vs. 14,5\%, p } \\
\geq 0.05 \\
\text { Hazard ratio (HR) of fracture (T2DM vs. } \\
\text { Non-DM): } \\
\text { Nonvertebral: HR 1.33 [1.00-1.76] } \\
\text { HR for treated diabetics vs. Controls: } \\
\text { Nonvertebral: HR 1.69 [1.16-2.46] } \\
\text { Hip: HR 1.26 [0.57-2.78] } \\
\text { Wrist: HR 2.14 [1.10-4.18] }\end{array}$ & $\begin{array}{l}\text { T2DM associated with: } \\
\text { - Increased risk of nonvertebral } \\
\text { fracture } \\
\text { - No increased risk of hip fracture } \\
\text { - No increased risk of wrist fracture } \\
\text { Newly diagnosed T2DM not } \\
\text { associated with increased risk. } \\
\text { Treatment with antidiabetics } \\
\text { associated with increased risk of all } \\
\text { fracture types }\end{array}$ \\
\hline [116] & Taylor et al. & $\begin{array}{l}6,787 \\
\text { (5.6\% T2DM; } \\
94.4 \% \text { non-DM) }\end{array}$ & $\begin{array}{l}\text { Age: mean } 74 \\
\text { US } \\
\text { Women }\end{array}$ & $\begin{array}{l}\text { *Adjusted for BMD, age, } \\
\text { weight, vision, height, } \\
\text { education level, digit symbol }\end{array}$ & $\begin{array}{l}\text { Hazard ratio (HR) of hip fracture (T2DM vs. } \\
\text { Non-DM): } \\
\text { In full population: HR } 1.83[1.34-2.50]^{*}\end{array}$ & $\begin{array}{l}\text { T2DM associated with: } \\
\text { - Increased risk of hip fracture } \\
\text { Effect of T2DM on fracture risk not }\end{array}$ \\
\hline
\end{tabular}




\begin{tabular}{|c|c|c|c|c|c|c|}
\hline & & $\frac{\text { Mean } 10.1 \mathrm{yrs}}{\underline{\mathrm{FU}}}$ & & $\begin{array}{l}\text { test score, walking speed, } \\
\text { parity, follow-up time }\end{array}$ & $\begin{array}{l}\text { In osteoporotic subpopulation: HR } 1.06 \\
\text { [0.52-2.16] } \\
\text { In non-osteoporotic subpopulation: HR } \\
\mathbf{1 . 9 7}[\mathbf{1 . 3 9 - 2 . 8 0 ]}\end{array}$ & present in osteoporotic subgroup \\
\hline [117] & $\begin{array}{l}\text { Ottenbacher } \\
\text { et al. }\end{array}$ & $\begin{array}{l}3,050 \\
\text { (690 T2DM; } \\
2,360 \text { non-DM) } \\
\underline{7 \text { yrs FU }}\end{array}$ & $\begin{array}{l}\text { Age: } \geq 65 \\
\text { Mexican Americans } \\
\text { (USA) } \\
57.9 \% \text { women }\end{array}$ & $\begin{array}{l}\text { Model 1: age, sex, smoking } \\
\text { status, BMI, history of stroke } \\
\text { Model 2: Model } 1+\text { measure } \\
\text { of lower body function, test } \\
\text { for distant vision }\end{array}$ & 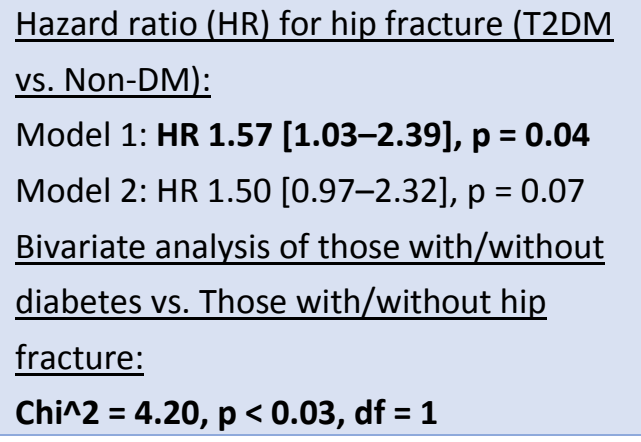 & $\begin{array}{l}\text { T2DM associated with: } \\
\text { - Increased risk of hip fracture in } \\
\text { two analyses. } \\
\text { Adjustment for previous tests for } \\
\underline{\text { lower body function and distant }} \\
\text { vision fully attenuated association. }\end{array}$ \\
\hline [118] & $\begin{array}{l}\text { Nicodemus } \\
\text { et al. }\end{array}$ & $\begin{array}{l}32,059 \\
(1,682 \text { T2DM; } \\
30,377 \text { non- } \\
\text { DM) } \\
\underline{306,900} \\
\text { person-yrs FU }\end{array}$ & $\begin{array}{l}\text { Age: mean } 61 \\
\text { US } \\
\text { Postmenopausal } \\
\text { women }\end{array}$ & $\begin{array}{l}\text { Age, smoking, estrogen use, } \\
\text { BMI, waist-to-hip ratio }\end{array}$ & $\begin{array}{l}\text { Relative risk (RR) for hip fracture } \\
\text { (compared to non-DM): } \\
\text { T2DM: RR 1.70 [1.21-2.38] } \\
\text { Diabetes duration 0-4 years: RR } 1.44 \\
\text { [0.79-2.63] } \\
\text { Diabetes duration 5-12 years: RR } 1.40 \\
\text { [0.77-2.57] } \\
\text { Diabetes duration 13-40 years: RR } 2.30 \\
\text { [1.39-3.81] } \\
\text { Nonobese (T2DM vs. non-DM): RR } 1.74 \\
\text { [1.14-2.67] } \\
\text { Never used estrogen (T2DM vs. non-DM): } \\
\text { RR 1.66 [1.10-2.51] } \\
\text { Ever estrogen users or obese (T2DM vs. } \\
\text { non-DM): } p>0.05\end{array}$ & $\begin{array}{l}\text { T2DM associated with: } \\
\text { - Increased risk of hip fracture. } \\
\text { Increased risk only found in } \\
\text { subgroups: } \\
\text { - Diabetes duration } \geq 13 \text { years } \\
\text { - Non-obese } \\
\text { - Never users of estrogen. }\end{array}$ \\
\hline
\end{tabular}




\begin{tabular}{|c|c|c|c|c|c|c|}
\hline & & Mean 5 yrs FU & & & $\begin{array}{l}\text { humerus: } \mathbf{R R} \mathbf{1 1 . 4} \text { [1.4-91.9] } \\
\text { Diabetes duration } \geq 10 \text { years, all fractures: } \\
\text { RR 2.9 [1.2-7.0] } \\
\text { Diabetes duration } \geq 10 \text { years, proximal } \\
\text { humerus: RR } \mathbf{1 1 . 0} \text { [2.3-51.8] } \\
\text { Diabetic retinopathy vs. no retinopathy: } \\
\text { Any fracture: } \mathbf{R R} \text { 4.6 [2.3-9.1] } \\
\text { Proximal humerus: RR } \mathbf{9 . 4} \text { [2.0-43.5] }\end{array}$ & $\begin{array}{l}\text { proximal humerus or 'any' fracture } \\
\text { Diabetes duration associated with } \\
\text { higher risks of: } \\
\text { - Any fracture and proximal } \\
\text { humerus fracture. } \\
\text { Diabetic retinopathy associated } \\
\text { with higher risks of: } \\
\text { - Any fracture and proximal } \\
\text { humerus fracture. }\end{array}$ \\
\hline [129] & Napoli et al. & $\begin{array}{l}\text { 5,995 } \\
\text { (881 T2DM; } \\
\text { 5,114 non-DM) } \\
\text { Mean } 9.1 \text { yrs } \\
\underline{\text { EU }}\end{array}$ & $\begin{array}{l}\text { Age: mean } 73 \\
\text { US } \\
\text { Men }\end{array}$ & $\begin{array}{l}\text { Age, race, clinic, hip BMD, } \\
\text { previous falls/fractures, BMI, } \\
\text { comorbiditiesa }{ }^{-} \text {TCA use, } \\
\text { smoking, grip strength, lower } \\
\text { body function }\end{array}$ & $\begin{array}{l}\text { Hazard ratio (HR) of incident fracture } \\
\text { (compared to NGM): } \\
\text { T2DM, all: HR 1.08 [0.91-1.29] (not } \\
\text { adjusted for hip BMD) } \\
\text { T2DM, all: HR } 1.30 \text { [1.09-1.54] (adjusted } \\
\text { for hip BMD) } \\
\text { T2DM, no insulin: HR } 1.00[0.80-1.25] \\
\text { T2DM, insulin: HR 1.74 [1.13-2.69] }\end{array}$ & $\begin{array}{l}\text { T2DM associated with: } \\
\text { - Increased risk of fracture only } \\
\text { when adjusting for hip BMD. } \\
\text { Increased risk not found in } \\
\text { subgroup of non-insulin users. } \\
\text { - No association with fracture } \\
\text { prevalence }\end{array}$ \\
\hline [14] & $\begin{array}{l}\text { Schwartz et } \\
\text { al. }\end{array}$ & $\begin{array}{l}9,548 \\
\text { (551 T2DM; } \\
8997 \text { non-DM) } \\
\text { Mean } 9.4 \text { yrs } \\
\underline{\text { FU }}\end{array}$ & $\begin{array}{l}\text { Age: } \geq 65 \\
\text { US } \\
\text { Women }\end{array}$ & $\begin{array}{l}\text { Age, BMI, calcaneal BMD, } \\
\text { height, height loss since age } \\
25, \text { contrast sensitivity, mother } \\
\text { fractured hip, medications }{ }^{\underline{a}} \text {, } \\
\text { recent falls, clinic, vision, } \\
\text { comorbidities } \underline{\text { a }} \text {, more }\end{array}$ & $\begin{array}{l}\text { Relative risk (RR) of fracture (T2DM vs. } \\
\text { non-DM): } \\
\text { Hip: RR 1.82 [1.24-2.69] } \\
\text { Proximal humerus: RR 1.94 [1.24-3.02] } \\
\text { All nonvertebral: RR 1.30 [1.10-1.53] } \\
\text { Distal forearm, ankle, foot, vertebral: } p> \\
0.05 \\
\text { Diabetes duration ( } \geq 14 \text { years vs. }<14 \\
\text { years): } \\
\text { Hip: } \text { RR } 2.40[1.55-3.71] \\
\text { Any other site: } p>0.05\end{array}$ & $\begin{array}{l}\text { T2DM associated with: } \\
\text { - Increased risk of hip, proximal } \\
\text { humerus and all nonvertebral } \\
\text { fracture } \\
\text { - No increased risk of distal } \\
\text { forearm, ankle, foot or vertebral } \\
\text { fracture. } \\
\text { Diabetes duration associated with: } \\
\text { - Increased hip fracture risk }\end{array}$ \\
\hline
\end{tabular}




\begin{tabular}{|c|c|c|c|c|c|c|}
\hline [119] & Lee et al. & $\begin{array}{l}662,628 \\
\text { All w/T2DM } \\
\text { Avg. FU } 3.43 \\
\frac{\text { yrs }}{\text { Total 2,272,930 }} \\
\text { person-yrs }\end{array}$ & $\begin{array}{l}\text { Age: } \geq 65 \text {, mean } 74- \\
76 \\
\text { US Citizens } \\
\text { Male veterans }\end{array}$ & $\begin{array}{l}\text { Comorbidities }{ }^{\mathrm{a}} \text {, race, } \mathrm{BMI} \text { and } \\
\text { age. }\end{array}$ & $\begin{array}{l}\text { Compared with HbA1c between } 7.5 \% \text { and } \\
\text { 8.5\%: } \\
\text { HbA1c < 6.5\%: HR } 1.08 \text { (Any fracture) } \\
\text { HbA1c < 6.5\%: HR } 1.13 \text { (hip fracture) } \\
\text { HbA1c > 9.5\%: HR } 1.1 \text { (hip fracture) }\end{array}$ & $\begin{array}{l}\text { Elevated } \mathrm{HbA} 1 \mathrm{c}(>9.5 \%) \text { associated } \\
\text { with: } \\
\text { - Increased risk of hip fracture. } \\
\text { Low } \mathrm{HbA} 1 \mathrm{c}(<6.5 \%) \text { associated } \\
\text { with: } \\
\text { - Increased risk of hip- and any } \\
\text { fracture }\end{array}$ \\
\hline [94] & Kabue et al. & $\begin{array}{l}\text { 120,256 } \\
\text { All w/T2DM } \\
\text { Unspecified } \\
\underline{\text { FU/OT }}\end{array}$ & $\begin{array}{l}\geq 65 \text {, mean } 73 \\
\text { US Citizens } \\
49.5 \% \text { women }\end{array}$ & Adjusted, unspecified & $\begin{array}{l}\text { No effect of } \mathrm{HbA} 1 \mathrm{c} \text { on fracture risk } \\
\text { Retinopathy: } \mathrm{OR}=\mathbf{1 . 7 0}[\mathbf{1 . 4 0 - 2 . 0 7 ]}(\mathrm{p}< \\
0.001) \text { for hip fracture }\end{array}$ & $\begin{array}{l}\text { Hip fracture risk: } \\
\text { - Associated with diabetic } \\
\text { retinopathy } \\
\text { - Not associated with HbA1c }\end{array}$ \\
\hline [97] & $\begin{array}{l}\text { Baltrusaitis } \\
\text { et al. }\end{array}$ & $\begin{array}{l}36,744 \\
(19,430 \text { T2DM; } \\
17,314 \text { non- } \\
\text { DM) } \\
\underline{7 \text { yr OT }}\end{array}$ & $\begin{array}{l}\text { Age: } \geq 65 \\
\text { US Citizens } \\
\text { Veterans } \\
1.7 \% \text { women }\end{array}$ & $\begin{array}{l}\text { Age, sex, race, BMI, s- } \\
\text { creatinine, hypoglycemia, prior } \\
\text { fracture, medications } \underline{\text { a }} \\
\text { comorbidities }\end{array}$ & 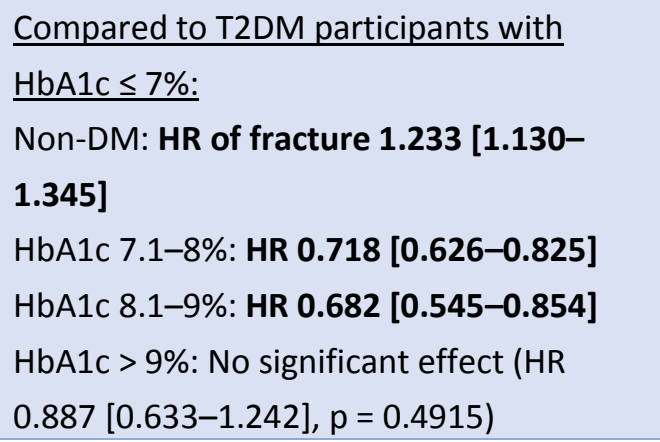 & $\begin{array}{l}\text { Risk of any fracture: } \\
\text { - Highest in non-diabetics } \\
\text { - Lowest in diabetics with HbA1c } \\
\text { between } 7.1-9 \%\end{array}$ \\
\hline [98] & $\begin{array}{l}\text { Martinez- } \\
\text { Huedo et al. }\end{array}$ & $\begin{array}{l}43,872 \\
(8,049 \text { T2DM; } \\
35,823 \text { non- } \\
\text { DM) }\end{array}$ & $\begin{array}{l}\text { Age: } \geq 65 \text {, mean } 76 \\
\text { Spanish } \\
83 \% \text { women } \\
\text { All: From database } \\
\text { of proximal } \\
\text { humerus fractures } \\
\text { with hospitalization } \\
\geq 24 \mathrm{~h}\end{array}$ & Age & $\begin{array}{l}\text { Incidence rate ratio (IRR) in T2DM vs. Non- } \\
\text { DM: } \\
\text { Proximal humerus fracture, men: IRR } 0.87 \\
{[0.82-0.93, p<0.01]} \\
\text { Proximal humerus fracture, women: IRR } \\
0.97[0.95-1.00, p<0.01]\end{array}$ & $\begin{array}{l}\text { T2DM associated with: } \\
\text { - Lower incidence rate of PHF in } \\
\text { men. } \\
\text { - No difference in women. }\end{array}$ \\
\hline
\end{tabular}




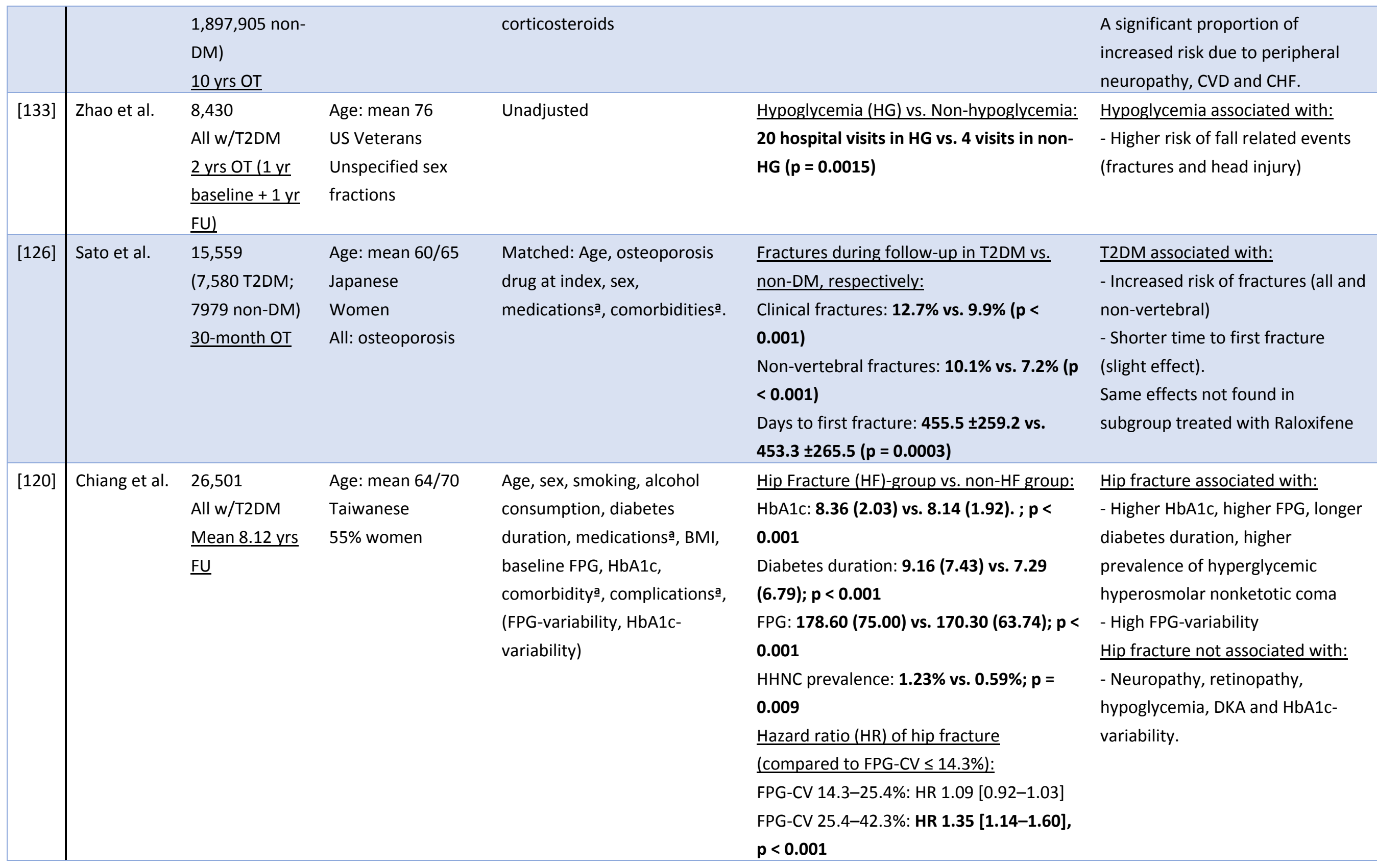




\begin{tabular}{|c|c|c|c|c|c|c|}
\hline & & & & & $\begin{array}{l}\text { FPG-CV >42.3\%: HR 1.27 [1.07-1.52], p } \\
<0.01\end{array}$ & \\
\hline [121] & Li et al. & $\begin{array}{l}20,025 \\
\text { All w/T2DM } \\
\text { Mean } 7.41 \mathrm{yrs} \\
\underline{\text { FU }}\end{array}$ & $\begin{array}{l}\text { Age: } \text { mean } 72-73 \\
\text { Taiwanese } \\
54.1 \% \text { women }\end{array}$ & $\begin{array}{l}\text { HRs adjusted for: age, sex, } \\
\text { smoking, alcohol consumption, } \\
\text { diabetes duration, glycemia, } \\
\text { comorbidities } \underline{\text { a }} \text {, medications } \underline{\text { a }} \\
\text { complications }\end{array}$ & $\begin{array}{l}\text { HF group vs. Non-HF group: } \\
\text { Diabetes duration - Mean (SD): } 9.21 \\
\text { (7.90) vs. } 8.16 \text { (7.55), p < } 0.001 \\
\text { Diabetic retinopathy: } 29.26 \% \text { vs. } 24.49 \% \text {, } \\
\text { p < } 0.001 \\
\text { Hypoglycemia: } 0.86 \% \text { vs. } 0.68 \%, p=0.52 \\
\text { Peripheral neuropathy: } 16.25 \% \text { vs. } \\
\text { 12.33\%, } p<0.001 \\
\text { Hazard ratio (HR) for hip fracture by } \\
\text { HbA1c: } \\
\text { HbA1c 9-10\%: HR } 1.24 \text { [1.02-1.49], } p< \\
0.05 \\
\text { HbA1c } \geq 10 \%: \text { HR } 1.32 \text { [1.09-1.58], } p< \\
0.01\end{array}$ & $\begin{array}{l}\text { Hip fracture associated with: } \\
\text { - Longer diabetes duration, } \\
\text { diabetic retinopathy and } \\
\text { peripheral neuropathy. } \\
\text { Hip fracture not associated with } \\
\text { hypoglycemia. } \\
\text { Higher risk of hip fracture in high } \\
\text { HbA1c (9-10\% and } \geq 10 \%) .\end{array}$ \\
\hline [134] & $\begin{array}{l}\text { Kachroo et } \\
\text { al. }\end{array}$ & $\begin{array}{l}1,147,937 \\
\text { All w/T2DM } \\
12 \text { months FU } \\
\end{array}$ & $\begin{array}{l}\text { Age: } \geq 65 \\
\text { US Citizens } \\
51.5 \% \text { women }\end{array}$ & $\begin{array}{l}\text { Age- and sex-matched. } \\
\text { Falls, antidiabetic drug, region, } \\
\text { healthcare plan, } \\
\text { comorbidities }\end{array}$ & $\begin{array}{l}\text { Fractures in hypoglycemia vs. non- } \\
\text { hypoglycemia group: } \\
\text { All ages: OR } 2.16 \text { [1.74-2.67] } \\
\text { Age }<75 \text { years: OR } 2.30 \text { [1.63-3.24] } \\
\text { Age } \geq 75 \text { years: OR } 2.07 \text { [1.58-2.72] }\end{array}$ & $\begin{array}{l}\text { Any fracture risk associated with: } \\
\text { - Hypoglycemia. }\end{array}$ \\
\hline [91] & Oei et al. & $\begin{array}{l}\text { 4,135 } \\
\text { (420 T2DM; } \\
3,715 \text { non-DM) } \\
\text { Mean } 12.2 \text { yrs } \\
\underline{\text { FU }}\end{array}$ & $\begin{array}{l}\text { Age: mean } 68-71 \\
\text { Dutch } \\
59.4 \% \text { women }\end{array}$ & $\begin{array}{l}\text { Age, sex, height, weight, } \\
\text { femoral neck BMD }\end{array}$ & $\begin{array}{l}\text { Hazard ratio (HR) for all fractures: } \\
\text { HbA1c } \geq 7.5 \% \text { vs. }<7.5 \%: \text { HR } 1.62[1.09- \\
2.40], p=0.02 \\
\text { HbA1c } \geq 7.5 \% \text { vs. Non-DM: HR } 1.47[1.12- \\
1.92], p=0.005 \\
\text { HbA1c }<7.5 \% \text { vs. Non-DM: HR } 0.91 \text { [0.67- } \\
1.23], p=0.54\end{array}$ & $\begin{array}{l}\text { Inadequately controlled T2DM } \\
\underline{\text { (HbA1c } \geq 7 \text { (compared to }} \\
\text { adequately controlled T2DM and } \\
\text { non-DM controls) associated with: } \\
\text { - Any fracture } \\
\text { - Wrist fracture risk } \\
\text { T2DM not associated with: }\end{array}$ \\
\hline
\end{tabular}




\begin{tabular}{|c|c|c|c|c|c|c|}
\hline & & & & & $\begin{array}{l}\text { HR for wrist fracture: } \\
\text { HbA1c } \geq 7.5 \% \text { vs. Non-DM: HR } 1.71 \text { [1.03- } \\
2.86], p=0.04\end{array}$ & $\begin{array}{l}\text { - Hip fracture risk (regardless of } \\
\text { HbA1c) }\end{array}$ \\
\hline [114] & $\begin{array}{l}\text { Lipscombe } \\
\text { et al. }\end{array}$ & $\begin{array}{l}598,812 \\
\text { 197,412 T2DM; } \\
401,400 \text { non- } \\
\text { DM) } \\
\underline{\text { Mean } 6.12 \text { yrs }} \\
\underline{\text { FU }}\end{array}$ & $\begin{array}{l}\text { Age: } \geq 66 \\
\text { Canadian } \\
49.4 \% \text { women }\end{array}$ & $\begin{array}{l}\text { *Age-group, comorbidities }{ }^{\underline{a}} \text {, } \\
\text { complications }{ }^{\mathrm{a}} \text {, medications }{ }^{\mathrm{a}} \text {, } \\
\text { history of BMD test } \\
\text { **Added insulin }^{*} \text { A*Added income quintile } \\
\text { (IncQ) } \\
* * * * \text { Added prevalent diabetes } \\
\text { and IncQ. }\end{array}$ & $\begin{array}{l}\text { Hazard ratio (HR) for hip fracture: } \\
\text { Women, T2DM vs. Non-DM: HR } 1.11 \\
\text { [1.08-1.15]* } \\
\text { Women, Prevalent vs. Incident DM: HR } \\
\text { 1.24 [1.13-1.37]** } \\
\text { Men, T2DM vs. Non-DM: HR } 1.18 \text { [1.12- } \\
\text { 1.24]*** } \\
\text { Men, Prevalent vs. Incident DM: HR } 1.37 \\
\text { [1.18-1.59] }]^{* * *}\end{array}$ & $\begin{array}{l}\text { T2DM associated with: } \\
\text { - Increased risk of hip fracture in } \\
\text { men and women. } * * * \\
\text { Higher risk with prevalent } \\
\underline{\text { compared to incident diabetes - }} \\
\underline{\text { hinting at a deleterious effect of }} \\
\underline{\text { diabetes duration. } * * * / * * * *}\end{array}$ \\
\hline \multicolumn{7}{|c|}{ Case-control Studies } \\
\hline [106] & $\begin{array}{l}\text { Lopez-de- } \\
\text { Andres et al. }\end{array}$ & $\begin{array}{l}432,760 \\
(92,182 \text { T2DM, } \\
340,578 \text { non- } \\
\text { DM) }\end{array}$ & $\begin{array}{l}\text { Age: mean } 81-82 \\
\text { Spanish } \\
77.4 \% \text { women } \\
\text { All: discharged after } \\
\text { hip fracture }\end{array}$ & $\begin{array}{l}\text { Year of discharge, age, } \\
\text { comorbiditya }, \text { complications, } \\
\text { type of repair, diabetes status } \\
\text { Age- and sex-matched }\end{array}$ & $\begin{array}{l}\text { Incidence rate ratio (IRR) of diabetic vs. } \\
\text { nondiabetic individuals: } \\
\text { Men: IRR } 1.08 \text { [1.06-1.09] } \\
\text { Women: IRR } 1.20 \text { [1.19-1.21] }\end{array}$ & $\begin{array}{l}\text { T2DM associated with: } \\
\text { - Increased risk of hip fracture (in } \\
\text { both sexes) }\end{array}$ \\
\hline [135] & Puar et al. & $\begin{array}{l}1,116 \\
\text { (558 hip } \\
\text { fractures; } \\
558 \text { non- }\end{array}$ & $\begin{array}{l}\text { Age: mean } 77 \\
\text { Singaporean } \\
73.3 \% \text { women } \\
\text { All: T2DM }\end{array}$ & $\begin{array}{l}\text { Age, sex, race, diabetes } \\
\text { duration, Charlson } \\
\text { Comorbidity Index, } \\
\text { complications } \text { /comorbiditiesa }\end{array}$ & $\begin{array}{l}\text { Odds ratio (OR) for hip fracture (compared } \\
\text { to } \mathrm{HbA} 1 \mathrm{c} \leq 8 \% \text { ): } \\
\text { HbA1c } \leq 6 \% \text { : OR } 3.03 \text { [2.03-4.52], p }<0.01 \\
\text { HbA1c 6.1-7.0\%: OR } 2.38 \text { [1.74-3.25], p < }\end{array}$ & $\begin{array}{l}\text { Higher risk of hip fracture in: } \\
\text { - HbA1c } \leq 6 \% \text { and } \\
\text { - HbA1c } 6.1-7.0 \% \\
\text { compared to } \mathrm{HbA} 1 \mathrm{c}>8 \%\end{array}$ \\
\hline
\end{tabular}




\begin{tabular}{|c|c|c|c|c|c|c|}
\hline & & fractures) & & high-risk medications. & $\begin{array}{l}0.01 \\
\text { HbA1c ratio (of fracture and non-fracture } \\
\text { group): } \\
0.78[0.72-0.85], p<0.01\end{array}$ & $\begin{array}{l}\text { Lower mean } \mathrm{HbA} 1 \mathrm{c} \text { in hip fracture } \\
\text { group. }\end{array}$ \\
\hline [130] & $\begin{array}{l}\text { Partanen et } \\
\text { al. }\end{array}$ & $\begin{array}{l}114 \\
\text { (74 T2DM; } \\
40 \text { non-DM) }\end{array}$ & $\begin{array}{l}\text { Age: mean } 73.7 \\
\text { Finnish } \\
\text { Postmenopausal } \\
\text { women }\end{array}$ & Unadjusted & $\begin{array}{l}\text { Use of oral antidiabetics in fracture group } \\
\text { vs. Non-fracture group: } \mathbf{p}=\mathbf{0 . 0 2 9}\end{array}$ & $\begin{array}{l}\text { Use of oral antidiabetics: } \\
\text { - Significantly more common in } \\
\text { fracture group }\end{array}$ \\
\hline \multicolumn{7}{|c|}{ Cross-sectional Studies } \\
\hline [69] & Sakane et al. & $\begin{array}{l}82 \\
\text { (17 T2DM; } \\
65 \text { non-DM) }\end{array}$ & $\begin{array}{l}\text { Age: median } 59 \\
\text { São Paolo. } \\
85 \% \text { women } \\
\text { All: Postsurgical } \\
\text { hypoparathyroidism }\end{array}$ & Unadjusted & $\begin{array}{l}\text { Patients } w / \text { fracture vs. non-fractured } \\
\text { group: } \\
\text { Mean glucose } 118 \mathrm{mg} / \mathrm{dL} \text { vs. } 95 \mathrm{mg} / \mathrm{dL} \text { ( } \mathrm{p}= \\
\mathbf{0 . 0 1 3} \text { ) } \\
\text { More diabetic/prediabetic patients ( } \mathrm{p}= \\
\mathbf{0 . 0 1 7 )}\end{array}$ & $\begin{array}{l}\text { Fracture correlated with: } \\
\text { - Diabetic status } \\
\text { - Glycemic control }\end{array}$ \\
\hline [93] & $\begin{array}{l}\text { Hatano et } \\
\text { al. }\end{array}$ & $\begin{array}{l}384 \\
\text { All w/T2DM }\end{array}$ & $\begin{array}{l}\text { Age: } \geq 65 \text {, mean } 87 \\
\text { Japanese } \\
76.6 \% \text { women }\end{array}$ & None & & $\begin{array}{l}\text { HbA1c not associated with fracture } \\
\underline{\text { risk }}\end{array}$ \\
\hline [38] & Raška et al. & $\begin{array}{l}112 \\
\text { All w/T2DM }\end{array}$ & $\begin{array}{l}\text { Age: mean } 65 \\
\text { Czech } \\
\text { Postmenopausal } \\
\text { women }\end{array}$ & & & $\begin{array}{l}\text { Previous vertebral fracture not } \\
\underline{\text { associated with FPG or } \mathrm{HbA1c}}\end{array}$ \\
\hline$[95]$ & Waard et al. & $\begin{array}{l}2,005 \\
\text { (400 T2DM; } \\
359 \text { IGM; } \\
1246 \text { healthy) }\end{array}$ & $\begin{array}{l}\text { Age: mean } 60-63 \\
\text { Netherlands } \\
49.6 \% \text { women }\end{array}$ & $\begin{array}{l}\text { Age, sex, level of education, } \\
\text { BMI, MVPA, comorbiditiesa, } \\
\text { smoking status, alcohol } \\
\text { consumption, medicationsa } \\
\text { cognitive function }\end{array}$ & $\begin{array}{l}\text { T2DM vs. normal glucose metabolism } \\
\text { (NGM): } \\
\text { Fracture prevalence: Lower in T2DM } \\
\text { (5.4\%) vs. NGM (10.3\%), p = } 0.006\end{array}$ & $\begin{array}{l}\text { T2DM associated with: } \\
\text { - Lower prevalence of fractures. } \\
\text { T2DM, high ( } \geq 7 \%) \text { HbA1c and long } \\
\text { ( } \geq 5 \text { yrs) diabetes duration: } \\
\text { - No effect on Odds ratio (OR) for } \\
\text { fracture }\end{array}$ \\
\hline
\end{tabular}


OBM Geriatrics 2020; 4(2), doi:10.21926/obm.geriatr.2002123

\begin{tabular}{|c|c|c|c|c|c|c|}
\hline & & & $35.4 \%$ women & & & \\
\hline [96] & Kilpadi et al. & $\begin{array}{l}296 \\
(122 \text { T2DM; } \\
174 \text { non-DM) }\end{array}$ & $\begin{array}{l}\text { Age: mean } 56 \\
\text { Southern Texas } \\
\text { Latinos } \\
56 \% \text { women }\end{array}$ & $\begin{array}{l}\text { Model for vertebral fracture } \\
\text { (ethnicity, diabetes, sex, and } \\
\text { propensity scorea) }\end{array}$ & $\begin{array}{l}\text { T2DM vs. Non-DM group: } \\
\text { Vertebral fracture: } 27.9 \% \text { vs. } 13.8 \% \text {, OR } \\
2.86[1.56-5.34], p<0.001\end{array}$ & $\begin{array}{l}\text { Vertebral fracture: } \\
\text { - Associated with T2DM. } \\
\text { - Not associated with HbA1c. }\end{array}$ \\
\hline [111] & $\begin{array}{l}\text { Carnevale et } \\
\text { al. }\end{array}$ & $\begin{array}{l}1,751 \\
\text { (974 T2DM; } \\
777 \text { non-DM) }\end{array}$ & $\begin{array}{l}\text { Age: mean } 64-65 \\
\text { Italian } \\
58.9 \% \text { women }\end{array}$ & Unadjusted & $\begin{array}{l}\text { T2DM vs. Non-DM: } \\
\text { Men, All fractures: } 30.99 \% \text { vs. } 21.70 \%, p= \\
0.009 \\
\text { Women, All fractures: } 28.78 \% \text { vs. } \\
19.926 \%, p<0.001 \\
\text { Both, All fractures: } 29.88 \% \text { vs. } 20.46 \%, p< \\
0.001 \\
\text { Women Femoral: } 0.41 \% \text { vs. } 1.845 \%, p= \\
0.032 \\
\text { Both, Humeral: } 3.80 \% \text { vs. } 2.06 \%, p=0.035 \\
\text { Both, Wrist: } 4.21 \% \text { vs. } 1.80 \%, p=0.004 \\
\text { Both; Radial, clinical vertebral, other: } p> \\
0.05\end{array}$ & $\begin{array}{l}\text { T2DM associated with: } \\
\text { - Higher prevalence of any } \\
\text { previous fracture in both men and } \\
\text { women } \\
\text { - Higher prevalence of humeral and } \\
\text { wrist fractures in total population } \\
\text { - Higher prevalence of femoral } \\
\text { fractures in females } \\
\text { T2DM not associated with: } \\
\text { - Fractures in any specific locations } \\
\text { in males }\end{array}$ \\
\hline [30] & $\begin{array}{l}\text { Yamamoto } \\
\text { et al. }\end{array}$ & $\begin{array}{l}495 \\
\text { (255 T2DM; } \\
240 \text { non-DM) }\end{array}$ & $\begin{array}{l}\text { Age: mean } 63 \\
\text { Japanese } \\
63 \% \\
\text { (postmenopausal) } \\
\text { women }\end{array}$ & & $\begin{array}{l}\text { No statistical testing! } \\
\text { Women (T2DM vs. Non-DM): } \mathbf{2 8 . 4 \%} \text { vs. } \\
\text { 21.7\% } \\
\text { Men (T2DM vs. Non-DM): } 41.7 \% \text { vs. } 61 \%\end{array}$ & $\begin{array}{l}\text { T2DM associated with: } \\
\text { - Higher prevalence of } V F \text { in } \\
\text { women. } \\
\text { - Lower prevalence of } V F \text { in men. }\end{array}$ \\
\hline [125] & Viegas et al. & $\begin{array}{l}148 \\
\text { All w/T2DM }\end{array}$ & $\begin{array}{l}\text { Age: mean } 62 \\
\text { Brazilian } \\
\text { Postmenopausal } \\
\text { women }\end{array}$ & $\begin{array}{l}\text { Age, T2DM duration, daily } \\
\text { calcium intake, diabetic } \\
\text { retinopathy, WHO BMD } \\
\text { classification, renal functiona, } \\
\text { PPG, HbA1c, s-triglycerides. }\end{array}$ & $\begin{array}{l}\text { Values according to prevalence of VF (VF } \\
\text { vs. No-VF): } \\
\text { PPG: } 141.79 \pm 74.78 \text { vs. } 178.15 \pm 86.64, p= \\
0.029 \\
\text { HbA1c: } 7.13 \pm 1.80 \text { vs. } 7.80 \pm 1.70, p= \\
0.049 \\
\text { Odss ratio (OR) for VF: }\end{array}$ & $\begin{array}{l}\text { Higher risk of vertebral fracture } \\
\text { associated with: } \\
\text { - Normal postprandial glucose (vs. } \\
\text { high PPG), longer diabetes } \\
\text { duration, presence of retinopathy } \\
\text { - Not nephropathy or peripheral } \\
\text { diabetic neuropathy }\end{array}$ \\
\hline
\end{tabular}




\begin{tabular}{|c|c|c|c|c|c|c|}
\hline & & & & & $\begin{array}{l}\text { Normal PPG (vs. abnormal): OR } 4.20 \\
{[1.45-12.33], p=0.008} \\
\text { Other associations: } \\
\text { Fracture increases with diabetes duration } \\
\text { ( } \geq 10 \text { years): } \mathbf{p}=\mathbf{0 . 0 3 7} \\
\text { Diabetic retinopathy increases fracture } \\
\text { risk: } \mathbf{p}=\mathbf{0 . 0 3 0}\end{array}$ & $\begin{array}{l}\text { Women with prevalent vertebral } \\
\text { fracture have: } \\
\text { - Lower postprandial glucose and } \\
\text { HbA1c }\end{array}$ \\
\hline [51] & Bulló et al. & $\begin{array}{l}251 \\
\text { (110 T2DM; } \\
141 \text { non-DM) }\end{array}$ & $\begin{array}{l}\text { Age: Mean } 67.70- \\
67.82 \\
\text { Spanish } \\
50.6 \% \text { women }\end{array}$ & Sex, age, BMI & $\begin{array}{l}\text { Odds ratio (OR) in T2DM (vs. non-DM): } \\
\text { Any fracture: } O R=0.393[0.167-0.965]\end{array}$ & $\frac{\text { T2DM associated with: }}{\text { Reduced risk of any fracture }}$ \\
\hline [89] & Sosa et al. & $\begin{array}{l}202 \\
\text { (101 T2DM; } \\
91 \text { non-DM) }\end{array}$ & $\begin{array}{l}\text { Age: } \geq 65 \\
\text { Spanish } \\
\text { Postmenopausal } \\
\text { women }\end{array}$ & Unadjusted & & $\begin{array}{l}\text { T2DM not associated with: } \\
\text { - Risk of vertebral fracture } \\
\text { - Risk of any fracture }\end{array}$ \\
\hline [81] & $\begin{array}{l}\text { Strotmeyer } \\
\text { et al. }\end{array}$ & $\begin{array}{l}\text { 2,979 } \\
\text { (566 T2DM; } \\
\text { 2,413 non-DM) }\end{array}$ & $\begin{array}{l}\text { Age: Mean } 73.3- \\
74.0 \\
\text { US } \\
51.1 \% \text { women }\end{array}$ & Unadjusted & $\begin{array}{l}\text { Fracture (T2DM vs. Non-DM): } \\
\text { White women: } \mathbf{2 1 . 4 \%} \text { vs. } \mathbf{3 4 . 1 \% ,} \mathbf{p}<\mathbf{0 . 0 5} \\
\text { Men and black women: } p>0.05\end{array}$ & $\begin{array}{l}\text { T2DM associated with: } \\
\text { - Reduced risk of fracture in white } \\
\text { women } \\
\text { - No effect on fracture risk in black } \\
\text { women or men }\end{array}$ \\
\hline [99] & $\begin{array}{l}\text { van Daele et } \\
\text { al. }\end{array}$ & $\begin{array}{l}\text { 5,931 } \\
\text { (578 T2DM; } \\
\text { 5,353 non-DM) }\end{array}$ & $\begin{array}{l}\text { Age: } \geq 55 \\
\text { Netherlands } \\
58.2 \% \text { women }\end{array}$ & Age, BMI & $\begin{array}{l}\text { Odds ratio (OR) of nonvertebral fracture } \\
\text { (compared to non-DM): } \\
\text { Women with T2DM: OR } 0.63 \text { [0.44-0.90] } \\
\text { Men with T2DM: OR 0.96 [0.60-1.52] } \\
\text { Newly diagnosed T2DM: } 0.62[0.35-1.10]\end{array}$ & $\begin{array}{l}\text { T2DM associated with: } \\
\text { - Reduced risk of nonvertebral } \\
\text { fracture in women. } \\
\text { - No altered risk in men. } \\
\text { - No altered risk in newly } \\
\text { diagnosed diabetics. }\end{array}$ \\
\hline [62] & $\begin{array}{l}\text { Yamamoto } \\
\text { et al. }\end{array}$ & $\begin{array}{l}996 \\
\text { (298 T2DM; } \\
698 \text { non-DM) }\end{array}$ & $\begin{array}{l}\text { Age: mean } 65-67 \\
\text { Japanese } \\
76.2 \% \text { women }\end{array}$ & $\begin{array}{l}\text { ORs adjusted for age, BMI and } \\
\text { L-BMD. }\end{array}$ & $\begin{array}{l}\text { Odds ratio of VF (T2DM vs. Non-DM): } \\
\text { Women: OR } 1.86 \text { [1.11-3.12], } p=0.019 \\
\text { Men: OR 4.73 [2.19-10.20], } p<0.001\end{array}$ & $\begin{array}{l}\text { Prevalent vertebral fracture } \\
\text { associated with: } \\
\text { - T2DM (in both sexes) } \\
\text { - Not diabetes duration or }\end{array}$ \\
\hline
\end{tabular}




\begin{tabular}{|c|c|c|c|c|c|c|}
\hline & & & & & & $\begin{array}{l}\text { complications } \\
\text { (neuropathy/retinopathy) } \\
\text { - Not fasting plasma glucose or } \\
\text { HbA1c }\end{array}$ \\
\hline [34] & Ardawi et al. & $\begin{array}{l}964 \\
\text { (482 T2DM; } \\
482 \text { non-DM) }\end{array}$ & $\begin{array}{l}\text { Age: Mean } 59.60 \\
\pm 7.90 \\
\text { Saudi Arabians } \\
\text { Postmenopausal } \\
\text { women }\end{array}$ & Unadjusted and age-adjusted & $\begin{array}{l}\text { Fracture prevalence (T2DM vs. non-DM): } \\
24.5 \% \text { vs. } 0.0 \%, p<0.0001 \text { (unadjusted) } \\
\text { Prevalent VF vs. no previous VF in those } \\
\text { with T2DM: } \\
\text { DM duration: } 11.58 \pm 3.53 \text { vs. } 9.51 \pm 3.92 \text {, } \\
\text { p<0.001 (adjusted } p=0.631 \text { ) } \\
\text { HbA1c: } 10.52 \pm 2.28 \text { vs. } 9.64 \pm 1.85, p< \\
0.001 \text { (adjusted } p=0.290 \text { ) }\end{array}$ & $\begin{array}{l}\text { Prevalent vertebral fracture } \\
\text { (assessed by } x \text {-ray) associated } \\
\text { with: } \\
\text { - T2DM } \\
\text { - Longer T2DM duration (fully } \\
\text { attenuated after adjustment for } \\
\text { age) } \\
\text { - Higher HbA1c (fully attenuated } \\
\text { after adjustment for age) }\end{array}$ \\
\hline
\end{tabular}

ạ: Parameters are summarized here (full list can be found in-article), s-: serum, p-: plasma, BMD: bone mineral density, BMI: body mass index, CHF: congestive heart failure, CKD: chronic kidney disease, CVD: cardiovascular disease, DM: diabetes mellitus, T1DM: type 1 DM, T2DM: type 2 DM, DXA: dual-energy X-ray absorptiometry, eGFR: estimated glomerular filtration rate, FPG: fasting plasma glucose, FPG-CV: FPGcoefficient of variation, FRAX: fracture risk assessment tool, FU: follow-up, HbA1c: hemoglobin A1c, HF: hip fracture, HHNC: hyperosmolar hyperglycemic nonketotic coma, HOMA: homeostatic model assessment, HOMA- $\beta$ : HOMA- $\beta$-cell, HOMA-IR: HOMA of insulin resistance, HR: hazard ratio, csHR: cause-specific H, sdHR: sub-distribution H, SHR: sub-hazard ratio, IGM: impaired glucose metabolism, IHD: ischemic heart disease, IRR: incidence rate ratio, mo: months, MOPF: major osteoporotic fracture, MVPA: moderate-to-vigorous physical activity, NGM: normal glucose metabolism, OPF: osteoporotic fracture, OR: odds ratio, PPG: postprandial glucose, PSN: peripheral sensory neuropathy, RR: relative risk, VF: vertebral fracture, vit-D: vitamin D, yr/yrs: year/years. 


\section{Additional Materials}

The following additional materials are uploaded at the page of this paper.

1. Table S1: Schematization of search terms used for PubMed.

2. Table S2: Search strings used for the respective databases.

\section{Author Contributions}

Authors ZAM and RV performed literature search, the article appraisal process and all data extraction. ZAM and RV devised the first draft of the manuscript. All additional authors reviewed all subsequent versions of the manuscript and provided detailed feedback for, and identified additional articles for inclusion.

\section{Funding}

The authors have received funding for this work by the Novo Nordisk Foundation (Steno Collaborative Grant no. NNF180C0052064).

\section{Competing Interests}

The authors have declared that no competing interests exist.

\section{References}

1. Fact sheet N. WHO | Ageing and health. 2015.

2. OECD/EU. Health at a Glance: Europe 2016 - State of Health in the EU Cycle. OECD Publishing; 2016.

3. Arias E, Xu J, Kochanek KD. United States Life Tables, 2016. National vital statistics reports: From the Centers for Disease Control and Prevention, National Center for Health Statistics, National Vital Statistics System. 2019.

4. International Diabetes F. IDF Diabetes Atlas, 8th ed. 2017.

5. International Osteoporosis F. The global burden of osteoporosis: A factsheet. International Osteoporosis Foundation. 2014.

6. Organisation WH. WHO Scientific Group on the Assessment of Osteoporosis at Primnary Health Care Level 2004 (Accessed: 16.12.2019) [cited 2019 Dec 16]. Available from: https://www.who.int/chp/topics/Osteoporosis.pdf.

7. Compston JE, McClung MR, Leslie WD. Osteoporosis. Lancet. 2019; 393: 364-376.

8. Schuit SCE, Van Der Klift M, Weel AEAM, De Laet CEDH, Burger H, Seeman E, et al. Fracture incidence and association with bone mineral density in elderly men and women: The Rotterdam Study. Bone. 2004; 34: 195-202.

9. DeFronzo RA, Ferrannini E, Zimmet P. International Textbook of Diabetes Mellitus. Vol. 1 (4th edition). Diabetes Care. 2010.

10. Longo M, Bellastella G, Maiorino MI, Meier JJ, Esposito K, Giugliano D. Diabetes and Aging: From Treatment Goals to Pharmacologic Therapy. Front Endocrinol. 2019; 10: 45. 
11. Marshall $\mathrm{D}$, Johnell $\mathrm{O}$, Wedel $\mathrm{H}$. Meta-analysis of how well measures of bone mineral density predict occurrence of osteoporotic fractures. BMJ. 1996; 312: 1254-1259.

12. Giangregorio LM, Leslie WD, Lix LM, Johansson $H$, Oden A, McCloskey $E$, et al. FRAX underestimates fracture risk in patients with diabetes. J Bone Miner Res. 2012; 27: 301-308.

13. Vestergaard $\mathrm{P}$. Discrepancies in bone mineral density and fracture risk in patients with type 1 and type 2 diabetes--a meta-analysis. Osteoporos Int. 2007; 18: 427-444.

14. Schwartz AV, Sellmeyer DE, Ensrud KE, Cauley JA, Tabor HK, Schreiner PJ, et al. Older women with diabetes have an increased risk of fracture: A prospective study. J Clin Endocrinol Metab. 2001; 86: 32-38.

15. Forsen L, Meyer HE, Midthjell K, Edna TH. Diabetes mellitus and the incidence of hip fracture: Results from the Nord-Trondelag Health Survey. Diabetologia. 1999; 42: 920-925.

16. Ma L, Oei L, Jiang L, Estrada K, Chen H, Wang Z, et al. Association between bone mineral density and type 2 diabetes mellitus: A meta-Analysis of observational studies. Eur J Epidemiol. 2012; 27: 319-332.

17. Hygum K, Starup-Linde J, Harslof T, Vestergaard P, Langdahl BL. MECHANISMS IN ENDOCRINOLOGY: Diabetes mellitus, a state of low bone turnover - a systematic review and meta-analysis. Eur J Endocrinol. 2017; 176: R137-R157.

18. Farr JN, Khosla S. Determinants of bone strength and quality in diabetes mellitus in humans. Bone. 2016; 82: 28-34.

19. Ferrari SL, Abrahamsen B, Napoli N, Akesson K, Chandran M, Eastell R, et al. Diagnosis and management of bone fragility in diabetes: An emerging challenge. Osteoporos Int. 2018; 29: 2585-2596.

20. PRISMA. PRISMA Statement 2019 [updated Dec 19; cited 2019 December]. Available from: www.prisma-statement.org.

21. Liberati A, Altman DG, Tetzlaff J, Mulrow C, Gotzsche PC, loannidis JP, et al. The PRISMA statement for reporting systematic reviews and meta-analyses of studies that evaluate healthcare interventions: Explanation and elaboration. BMJ. 2009; 339: b2700.

22. Dennison EM, Syddall HE, Sayer AA, Craighead S, Phillips DIW, Cooper C. Type 2 diabetes mellitus is associated with increased axial bone density in men and women from the Hertfordshire Cohort Study: Evidence for an indirect effect of insulin resistance? Diabetologia. 2004; 47: 1963-1968.

23. Sahin G, Polat G, Bagis S, Milcan A, Erdogan C. Study of axial bone mineral density in postmenopausal women with diffuse idiopathic skeletal hyperostosis related to type 2 diabetes mellitus. J Womens Health (Larchmt). 2002; 11: 801-804.

24. Shetty S, Kapoor N, Bondu JD, Thomas N, Paul TV. Bone turnover markers: Emerging tool in the management of osteoporosis. Indian J Endocrinol Metab. 2016; 20: 846-852.

25. van Summeren M, Braam L, Noirt F, Kuis W, Vermeer C. Pronounced elevation of undercarboxylated osteocalcin in healthy children. Pediatr Res. 2007; 61: 366-370.

26. Liu M, Lu Y, Cheng X, Ma L, Miao X, Li N, et al. Relationship between abnormal glucose metabolism and osteoporosis in han Chinese men over the age of 50 years. Clin Interv Aging. 2019; 14: 445-451.

27. Shou Z, Jin X, Bian P, Li X, Chen J. Reference intervals of $\beta$-C-terminal telopeptide of type I collagen, procollagen type I $\mathrm{N}$-terminal propeptide and osteocalcin for very elderly Chinese men. Geriatr Gerontol Int. 2017; 17: 773-778. 
28. Furst JR, Bandeira LC, Fan WW, Agarwal S, Nishiyama KK, McMahon DJ, et al. Advanced glycation endproducts and bone material strength in type 2 diabetes. J Clin Endocrinol Metab. 2016; 101: 2502-2510.

29. Yeap BB, Alfonso H, Paul Chubb SA, Gauci R, Byrnes E, Beilby JP, et al. Higher serum undercarboxylated osteocalcin and other bone turnover markers are associated with reduced diabetes risk and lower estradiol concentrations in older men. J Clin Endocrinol Metab. 2015; 100: 63-71.

30. Yamamoto M, Yamaguchi T, Nawata K, Yamauchi M, Sugimoto T. Decreased PTH levels accompanied by low bone formation are associated with vertebral fractures in postmenopausal women with type 2 diabetes. J Clin Endocrinol Metab. 2012; 97: 1277-1284.

31. Dobnig H, Piswanger-Sölkner JC, Roth M, Obermayer-Pietsch B, Tiran A, Strele A, et al. Type 2 diabetes mellitus in nursing home patients: Effects on bone turnover, bone mass, and fracture risk. J Clin Endocrinol Metab. 2006; 91: 3355-3363.

32. Gerdhem P, Isaksson A, Åkesson K, Obrant KJ. Increased bone density and decreased bone turnover, but no evident alteration of fracture susceptibility in elderly women with diabetes mellitus. Osteoporos Int. 2005; 16: 1506-1512.

33. Garcia-Martin A, Rozas-Moreno P, Reyes-Garcia R, Morales-Santana S, Garcia-Fontana B, Garcia-Salcedo JA, et al. Circulating levels of sclerostin are increased in patients with type 2 diabetes mellitus. J Clin Endocrinol Metab. 2012; 97: 234-241.

34. Ardawi MS, Akhbar DH, Alshaikh A, Ahmed MM, Qari MH, Rouzi AA, et al. Increased serum sclerostin and decreased serum IGF-1 are associated with vertebral fractures among postmenopausal women with type-2 diabetes. Bone. 2013; 56: 355-362.

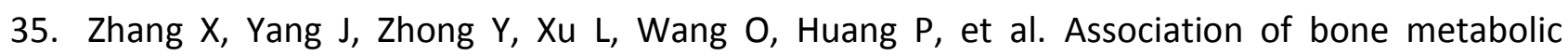
markers with diabetic retinopathy and diabetic macular edema in elderly Chinese individuals with type 2 diabetes mellitus. Am J Med Sci. 2017; 354: 355-361.

36. Osima M, Kral R, Borgen TT, HøgestøI IK, Joakimsen RM, Eriksen EF, et al. Women with type 2 diabetes mellitus have lower cortical porosity of the proximal femoral shaft using lowresolution $\mathrm{CT}$ than nondiabetic women, and increasing glucose is associated with reduced cortical porosity. Bone. 2017; 97: 252-260.

37. Maagensen H, Junker AE, Jørgensen NR, Gluud LL, Knop FK, VilsbøII T. Bone turnover markers in patients with nonalcoholic fatty liver disease and/or type 2 diabetes during oral glucose and isoglycemic intravenous glucose. J Clin Endocrinol Metab. 2018; 103: 2042-2049.

38. Raška Jr I, Rašková M, Zikán V, Škrha J, Raška I, Jr. Prevalence and risk factors of osteoporosis in postmenopausal women with type 2 diabetes mellitus. Cent Eur J Public Health.. 2017; 25: 3-10.

39. Iki M, Fujita Y, Kouda K, Yura A, Tachiki T, Tamaki J, et al. Hyperglycemia is associated with increased bone mineral density and decreased trabecular bone score in elderly Japanese men: The Fujiwara-kyo osteoporosis risk in men (FORMEN) study. Bone. 2017; 105: 18-25.

40. Chailurkit LO, Chanprasertyothin S, Rajatanavin R, Ongphiphadhanakul B. Reduced attenuation of bone resorption after oral glucose in type 2 diabetes. Clin Endocrinol (Oxf). 2008; 68: 858-862.

41. Zhou Y, Li Y, Zhang D, Wang J, Yang H. Prevalence and predictors of osteopenia and osteoporosis in postmenopausal Chinese women with type 2 diabetes. Diabetes Res Clin Pract. 2010; 90: 261-269. 
42. Mitchell A, Fall T, Melhus $H$, Wolk A, Michaëlsson $K$, Byberg L. Type 2 diabetes in relation to hip bone density, area, and bone turnover in Swedish men and women: A cross-sectional study. Calcif Tissue Int. 2018; 103: 501-511.

43. Yano S, Nabika T, Nagai A, Hamano T, Yamasaki $M$, Isomura $M$, et al. Interrelationship between glucose metabolism and undercarboxylated osteocalcin: A cross-sectional study in a community-dwelling population. Asia Pac J Clin Nutr. 2015; 24: 489.

44. Iki M, Tamaki J, Fujita Y, Kouda K, Yura A, Kadowaki E, et al. Serum undercarboxylated osteocalcin levels are inversely associated with glycemic status and insulin resistance in an elderly Japanese male population: Fujiwara-kyo Osteoporosis Risk in Men (FORMEN) Study. Osteoporos Int. 2012; 23: 761-770.

45. Hannemann A, Friedrich N, Spielhagen C, Rettig R, Ittermann T, Nauck M, et al. Reference intervals for serum osteocalcin concentrations in adult men and women from the study of health in Pomerania. BMC Endocr Disord. 2013; 13: 11.

46. Rianon NJ, Smith SM, Lee MJ, Pervin H, Musgrave P, Watt GP, et al. Glycemic control and bone turnover in older Mexican Americans with type 2 diabetes. J Osteoporos. 2018; 2018: 7153021.

47. Bulló M, Moreno-Navarrete JM, Fernández-Real JM, Salas-Salvadó J. Total and undercarboxylated osteocalcin predict changes in insulin sensitivity and $\beta$ cell function in elderly men at high cardiovascular risk. Am J Clin Nutr. 2012; 95: 249-255.

48. Xia J, Zhong Y, Huang G, Chen Y, Shi H, Zhang Z. The relationship between insulin resistance and osteoporosis in elderly male type 2 diabetes mellitus and diabetic nephropathy. Ann Endocrinol (Paris). 2012; 73: 546-551.

49. Feldbrin Z, Shargorodsky M. Bone remodelling markers in hypertensive patients with and without diabetes mellitus: Link between bone and glucose metabolism. Diabetes Metab Res Rev. 2015; 31: 752-757.

50. Rasul S, Ilhan A, Wagner L, Luger A, Kautzky-Willer A. Diabetic polyneuropathy relates to bone metabolism and markers of bone turnover in elderly patients with type 2 diabetes: Greater effects in male patients. Gend Med. 2012; 9: 187-196.

51. Bulló M, Garcia-Aloy M, Basora J, Covas MI, Salas-Salvado J. Bone quantitative ultrasound measurements in relation to the metabolic syndrome and type 2 diabetes mellitus in a cohort of elderly subjects at high risk of cardiovascular disease from the predimed study. J Nutr Health Aging. 2011; 15: 939-944.

52. Schwartz AV, Margolis KL, Sellmeyer DE, Vittinghoff E, Ambrosius WT, Bonds DE, et al. Intensive glycemic control is not associated with fractures or falls in the ACCORD randomized trial. Diabetes Care. 2012; 35: 1525-1531.

53. Tuominen JT, Impivaara O, Puukka $P$, Rǒnnemaa $T$. Bone mineral density in patients with type 1 and type 2 diabetes. Diabetes Care. 1999; 22: 1196-1200.

54. Cherif R, Mahjoub F, Sahli H, Cheour E, Sakly M, Attia N. Clinical and body composition predictors of bone turnover and mineral content in obese postmenopausal women. Clin Rheumatol. 2019; 38: 739-747.

55. Razi F, Esmaili M, Esfahani EN, Yaghmaei P, Qorbani M, Mohammadi Z, et al. Bone structure and turnover in postmenopausal women with type 2 diabetes mellitus. Menopause. 2016; 23: 280-285. 
56. Amer MS, Ali-Labib R, Farid TM, Rasheedy D, Tolba MF. Link between vitamin B12, type 2 diabetes mellitus, and bone mineral density in elderly patients. J Clin Gerontol Geriatr. 2015; 6: 120-124.

57. Akeroyd JM, Suarez EA, Bartali B, Chiu GR, Yang M, Schwartz AV, et al. Differences in skeletal and non-skeletal factors in a diverse sample of men with and without type 2 diabetes mellitus. J Diabetes Complications. 2014; 28: 679-683.

58. Kim JH, Choi HJ, Ku EJ, Kim KM, Kim SW, Cho NH, et al. Trabecular bone score as an indicator for skeletal deterioration in diabetes. J Clin Endocrinol Metab. 2015; 100: 475-482.

59. Cui R, Zhou L, Li Z, Li Q, Qi Z, Zhang J. Assessment risk of osteoporosis in Chinese people: Relationship among body mass index, serum lipid profiles, blood glucose, and bone mineral density. Clin Interv Aging. 2016; 11: 887-895.

60. Xu L, Cheng M, Liu X, Shan P, Gao H. Bone mineral density and its related factors in elderly male Chinese patients with type 2 diabetes. Arch Med Res. 2007; 38: 259-264.

61. Sert M, Tetiker T, Kirim S, Soyupak S, Canataroğlu A, Koçak M. Type 2 diabetes mellitus and osteopenia: Is there an association? Acta Diabetol. 2003; 40: 105-108.

62. Yamamoto $M$, Yamaguchi T, Yamauchi M, Kaji H, Sugimoto T. Diabetic patients have an increased risk of vertebral fractures independent of BMD or diabetic complications. J Bone Miner Res. 2009; 24: 702-709.

63. Sun Q, Zheng Y, Chen K, Yan W, Lu J, Dou J, et al. Osteopenia is associated with glycemic levels and blood pressure in Chinese postmenopausal women: A cross-sectional study. Clin Exp Med. 2017; 17: 85-91.

64. Shan PF, Wu XP, Zhang H, Cao XZ, Yuan LQ, Liao EY. Age-related bone mineral density, osteoporosis rate and risk of vertebral fracture in mainland Chinese women with type 2 diabetes mellitus. J Endocrinol Invest. 2011; 34: 190-196.

65. Mitama Y, Fujiwara S, Yoneda M, Kira S, Kohno N. Association of type 2 diabetes and an inflammatory marker with incident bone fracture among a Japanese cohort. J Diabetes Investig. 2017; 8: 709-715.

66. Silva BC, Leslie WD, Resch H, Lamy O, Lesnyak O, Binkley N, et al. Trabecular bone score: A noninvasive analytical method based upon the DXA image. J Bone Miner Res. 2014; 29: 518530.

67. McCloskey EV, Odén A, Harvey NC, Leslie WD, Hans D, Johansson H, et al. A meta-analysis of trabecular bone score in fracture risk prediction and its relationship to FRAX. J Bone Miner Res. 2016; 31: 940-948.

68. Hans D, Goertzen AL, Krieg MA, Leslie WD. Bone microarchitecture assessed by TBS predicts osteoporotic fractures independent of bone density: The manitoba study. J Bone Miner Res. 2011; 26: 2762-2769.

69. Sakane EN, Camargo Vieira MC, Lazaretti-Castro M, Maeda SS. Predictors of poor bone microarchitecture assessed by trabecular bone score (TBS) in postsurgical hypoparathyroidism. J Clin Endocrinol Metab. 2019; 104: 5795-5803.

70. Baleanu F, Bergmann P, Hambye AS, Dekelver C, Iconaru L, Cappelle SI, et al. Assessment of bone quality with trabecular bone score in type 2 diabetes mellitus: A study from the FRISBEE cohort. Int J Clin Pract. 2019; 73: e13347.

71. Leslie WD, Aubry-Rozier B, Lamy O, Hans D. TBS (trabecular bone score) and diabetes-related fracture risk. J Clin Endocrinol Metab. 2013; 98: 602-609. 
72. Ho-Pham LT, Nguyen TV. Association between trabecular bone score and type 2 diabetes: A quantitative update of evidence. Osteoporos Int. 2019; 30: 2079-2085.

73. Mikolajewicz N, Bishop N, Burghardt AJ, Folkestad L, Hall A, Kozloff KM, et al. HR-pQCT measures of bone microarchitecture predict fracture: Systematic review and meta-analysis. J Bone Miner Res. 2020; 35: 446-459.

74. Cheung AM, Adachi JD, Hanley DA, Kendler DL, Davison KS, Josse R, et al. High-resolution peripheral quantitative computed tomography for the assessment of bone strength and structure: A review by the canadian bone strength working group. Curr Osteoporos Rep. 2013; 11: 136-146.

75. Napoli N, Schwartz AV, Schafer AL, Vittinghoff E, Cawthon PM, Parimi N, et al. Vertebral fracture risk in diabetic elderly men: The MrOS study. J Bone Miner Res. 2018; 33: 63-69.

76. Ho-Pham LT, Chau PMN, Do AT, Nguyen HC, Nguyen TV. Type 2 diabetes is associated with higher trabecular bone density but lower cortical bone density: The Vietnam Osteoporosis Study. Osteoporo Int. 2018; 29: 2059-2067.

77. Burghardt AJ, Issever AS, Schwartz AV, Davis KA, Masharani U, Majumdar S, et al. Highresolution peripheral quantitative computed tomographic imaging of cortical and trabecular bone microarchitecture in patients with type 2 diabetes mellitus. J Clin Endocrinol Metab. 2010; 95: 5045-5055.

78. Sheu Y, Amati F, Schwartz AV, Danielson ME, Li X, Boudreau R, et al. Vertebral bone marrow fat, bone mineral density and diabetes: The Osteoporotic Fractures in Men (MrOS) study. Bone. 2017; 97: 299-305.

79. de Waard EAC, de Jong JJA, Koster A, Savelberg HHCM, van Geel TA, Houben AJHM, et al. The association between diabetes status, $\mathrm{HbA1c}$, diabetes duration, microvascular disease, and bone quality of the distal radius and tibia as measured with high-resolution peripheral quantitative computed tomography-The Maastricht Study. Osteoporos Int. 2018; 29: 27252738.

80. Strotmeyer ES, Cauley JA, Schwartz AV, Nevitt MC, Resnick HE, Bauer DC, et al. Nontraumatic fracture risk with diabetes mellitus and impaired fasting glucose in older white and black adults: The health, aging, and body composition study. Arch Intern Med. 2005; 165: 16121617.

81. Strotmeyer ES, Cauley JA, Schwartz AV, Nevitt MC, Resnick HE, Zmuda JM, et al. Diabetes is associated independently of body composition with BMD and bone volume in older white and black men and women: The health, aging, and body composition study. J Bone Miner Res. 2004; 19: 1084-1091.

82. Nakamura M, Inaba M, Yamada S, Ozaki E, Maruo S, Okuno S, et al. Association of decreased handgrip strength with reduced cortical thickness in Japanese female patients with type 2 diabetes mellitus. Sci Rep. 2018; 8: 10767.

83. Pritchard JM, Giangregorio LM, Atkinson SA, Beattie KA, Inglis D, loannidis G, et al. Association of larger holes in the trabecular bone at the distal radius in postmenopausal women with type 2 diabetes mellitus compared to controls. Arthritis Care Res (Hoboken). 2012; 64: 83-91.

84. Pritchard JM, Giangregorio LM, Atkinson SA, Beattie KA, Inglis D, loannidis $G$, et al. Changes in trabecular bone microarchitecture in postmenopausal women with and without type 2 diabetes: A two year longitudinal study. BMC Musculoskelet Disord. 2013; 14: 114. 
85. Napoli N, Conte C, Pedone C, Strotmeyer ES, Barbour KE, Black DM, et al. Effect of insulin resistance on BMD and fracture risk in older adults. J Clin Endocrinol Metab. 2019; 104: 33033310.

86. Jang M, Kim H, Lea S, Oh S, Kim JS, Oh B. Effect of duration of diabetes on bone mineral density: A population study on East Asian males. BMC Endocr Disord. 2018; 18: 61.

87. Leslie WD, Morin SN, Majumdar SR, Lix LM. Effects of obesity and diabetes on rate of bone density loss. Osteoporos Int. 2018; 29: 61-67.

88. Xu H, Wang Z, Li X, Fan M, Bao C, Yang R, et al. Osteoporosis and osteopenia among patients with type 2 diabetes aged $\geq 50$ : Role of sex and clinical characteristics. J Clin Densitom. 2020; 23: 29-36.

89. Sosa M, Saavedra P, Jódar E, Lozano-Tonkin C, Quesada JM, Torrijos A, et al. Bone mineral density and risk of fractures in aging, obese post-menopausal women with type 2 diabetes. The GIUMO Study. Aging Clin Exp Res. 2009; 21: 27-32.

90. Iki M, Fujita Y, Kouda K, Yura A, Tachiki T, Tamaki J, et al. Hyperglycemic status is associated with an elevated risk of osteoporotic fracture in community-dwelling elderly Japanese men: The Fujiwara-kyo osteoporosis risk in men (FORMEN) cohort study. Bone. 2019; 121: 100-106.

91. Oei L, Zillikens MC, Dehghan A, Buitendijk GHS, Castaño-Betancourt MC, Estrada K, et al. High bone mineral density and fracture risk in type 2 diabetes as skeletal complications of inadequate glucose control: The Rotterdam study. Diabetes Care. 2013; 36: 1619-1628.

92. Ivers RQ, Cumming RG, Mltchell $P$, Peduto AJ. Diabetes and risk of fracture: The blue mountains eye study. Diabetes Care. 2001; 24: 1198-1203.

93. Hatano $\mathrm{Y}$, Araki A, Matsumoto M, Ishibashi S. Low hemoglobin A1c and low body mass index are associated with dementia and activities of daily living disability among Japanese nursing home residents with diabetes. Geriatr Gerontol Int. 2019; 19: 854-860.

94. Kabue S, Liu V, Dyer W, Raebel M, Nichols G, Schmittdiel J. Identifying common predictors of multiple adverse outcomes among elderly adults with type-2 diabetes. Med Care. 2019; 57: 702-709.

95. de Waard EAC, Koster A, Melai T, van Geel TA, Henry RMA, Schram MT, et al. The association between glucose metabolism status, diabetes severity and a history of fractures and recent falls in participants of 50 years and older-the Maastricht Study. Osteoporos Int. 2016; 27: 3207-3216.

96. Kilpadi KL, EIDabaje R, Schmitz JE, Ehler B, Thames TA, Joshi AP, et al. Type 2 diabetes is associated with vertebral fractures in a sample of clinic- and hospital-based Latinos. J Immigr Minor Health. 2014; 16: 440-449.

97. Baltrusaitis SL, Grabarczyk T. Glycemic control and 10-year odds of all-cause fractures in elderly veterans with type 2 diabetes. Diabetes Res Clin Pract. 2019; 151: 46-55.

98. Martinez-Huedo MA, Jiménez-García R, Mora-Zamorano E, Hernández-Barrera $V$, VillanuevaMartinez $M$, Lopez-De-Andres A. Trends in incidence of proximal humerus fractures, surgical procedures and outcomes among elderly hospitalized patients with and without type 2 diabetes in Spain (2001-2013). BMC Musculoskelet Disord. 2017; 18: 522.

99. Van Daele PLA, Stolk RP, Burger H, Algra D, Grobbee DE, Hofman A, et al. Bone density in noninsulin-dependent diabetes mellitus. The Rotterdam study. Ann Intern Med. 1995; 122: 409414. 
100. Tebé C, Martínez-Laguna D, Carbonell-Abella C, Reyes C, Moreno V, Diez-Perez A, et al. The association between type 2 diabetes mellitus, hip fracture, and post-hip fracture mortality: $A$ multi-state cohort analysis. Osteoporos Int. 2019; 30: 2407-2415.

101. Tebé C, Martinez-Laguna D, Moreno V, Cooper C, Diez-Perez A, Collins GS, et al. Differential mortality and the excess rates of hip fracture associated with type 2 diabetes: Accounting for competing risks in fracture prediction matters. J Bone Miner Res. 2018; 33: 1417-1421.

102. Lee RH, Sloane R, Pieper C, Lyles KW, Adler RA, Van Houtven C, et al. Clinical fractures among older men with diabetes are mediated by diabetic complications. J Clin Endocrinol Metab. 2018; 103: 281-287.

103. Kim SH, Kim YM, Yoo JS, Choe EY, Kim TH, Won YJ. Increased risk of hip fractures in Korean patients with type 2 diabetes: A 6-year nationwide population-based study. J Bone Miner Metab. 2017; 35: 623-629.

104. Dytfeld J, Michalak M. Type 2 diabetes and risk of low-energy fractures in postmenopausal women: Meta-analysis of observational studies. Aging Clin Exp Res. 2017; 29: 301-309.

105. Majumdar SR, Leslie WD, Lix LM, Morin SN, Johansson H, Oden A, et al. Longer duration of diabetes strongly impacts fracture risk assessment: The Manitoba BMD cohort. J Clin Endocrinol Metab. 2016; 101: 4489-4496.

106. Lopez-de-Andrés A, Jiménez-García R, Jiménez-Trujillo I, Hernández-Barrera V, de MiguelYanes JM, Méndez-Bailón $M$, et al. Incidence, surgical procedures, and outcomes of hip fracture among elderly type 2 diabetic and non-diabetic patients in Spain (2004-2013). Osteoporos Int. 2016; 27: 605-616.

107. Hamilton E, Davis WA, Bruce DG, Davis TME. Influence of premature mortality on the link between type 2 diabetes and hip fracture: The fremantle diabetes study. J Clin Endocrinol Metab. 2017; 102: 551-559.

108. Lee RH, Pieper CF, Colon-Emeric C. Functional Impairments Mediate association between clinical fracture risk and type 2 diabetes mellitus in older women. J Am Geriatr Soc. 2015; 63: 1546-1551.

109. Reyes $C$, Estrada $P$, Nogués $X$, Orozco $P$, Cooper $C$, Díez-Pérez A, et al. The impact of common co-morbidities (as measured using the Charlson index) on hip fracture risk in elderly men: $A$ population-based cohort study. Osteoporos Int. 2014; 25: 1751-1758.

110. Hothersall EJ, Livingstone SJ, Looker HC, Ahmed SF, Cleland S, Leese GP, et al. Contemporary risk of hip fracture in type 1 and type 2 diabetes: A national registry study from Scotland. J Bone Miner Res. 2014; 29: 1054-1060.

111. Carnevale V, Morano S, Fontana A, Annese MA, Fallarino M, Filardi T, et al. Assessment of fracture risk by the FRAX algorithm in men and women with and without type 2 diabetes mellitus: A cross-sectional study. Diabetes Metab Res Rev. 2014; 30: 313-322.

112. Hippisley-Cox J, Coupland C. Derivation and validation of updated QFracture algorithm to predict risk of osteoporotic fracture in primary care in the United Kingdom: Prospective open cohort study. BMJ. 2012; 344: e3427.

113. Schwartz AV, Vittinghoff E, Bauer DC, Hillier TA, Strotmeyer ES, Ensrud KE, et al. Association of BMD and FRAX score with risk of fracture in older adults with type 2 diabetes. JAMA. 2011; 305: 2184-2192.

114. Lipscombe LL, Jamal SA, Booth GL, Hawker GA. The risk of hip fractures in older individuals with diabetes: A population-based study. Diabetes Care. 2007; 30: 835-841. 
115. Janghorbani M, Feskanich D, Willett WC, Hu F. Prospective study of diabetes and risk of hip fracture: The nurses' health study. Diabetes Care. 2006; 29: 1573-1578.

116. Taylor BC, Schreiner PJ, Stone KL, Fink HA, Cummings SR, Nevitt MC, et al. Long-term prediction of incident hip fracture risk in elderly white women: Study of osteoporotic fractures. J Am Geriatr Soc. 2004; 52: 1479-1486.

117. Ottenbacher KJ, Ostir GV, Kristen Peek M, Goodwin JS, Markides KS. Diabetes mellitus as a risk factor for hip fracture in Mexican American older adults. J Gerontol A Biol Sci Med Sci. 2002; 57: M648-M653.

118. Nicodemus KK, Folsom AR. Type 1 and type 2 diabetes and incident hip fractures in postmenopausal women. Diabetes Care. 2001; 24: 1192-1197.

119. Lee RH, Sloane R, Pieper C, Lyles KW, Adler RA, Van Houtven C, et al. Glycemic control and insulin treatment alter fracture risk in older men with type 2 diabetes mellitus. J Bone Miner Res. 2019; 34: 2045-2051.

120. Chiang JIH, Li TC, Li Cl, Liu CS, Meng NH, Lin WY, et al. Visit-to-visit variation of fasting plasma glucose is a predictor of hip fracture in older persons with type 2 diabetes: The Taiwan Diabetes Study. Osteoporos Int. 2016; 27: 3587-3597.

121. Li Cl, Liu CS, Lin WY, Meng NH, Chen CC, Yang SY, et al. Glycated hemoglobin level and risk of hip fracture in older people with type 2 diabetes: A competing risk analysis of Taiwan Diabetes Cohort Study. J Bone Miner Res. 2015; 30: 1338-1346.

122. Conway BN, Long DM, Figaro MK, May ME. Glycemic control and fracture risk in elderly patients with diabetes. Diabetes Res Clin Pract. 2016; 115: 47-53.

123. De Liefde II, Van Der Klift M, De Laet CEDH, Van Daele PLA, Hofman A, Pols HAP. Bone mineral density and fracture risk in type-2 diabetes mellitus: The Rotterdam Study. Osteoporos Int. 2005; 16: 1713-1720.

124. Wallander M, Axelsson KF, Nilsson AG, Lundh D, Lorentzon M. Type 2 diabetes and risk of hip fractures and non-skeletal fall injuries in the elderly: A study from the fractures and fall injuries in the elderly cohort (FRAILCO). J Bone Miner Res. 2017; 32: 449-460.

125. Viégas M, Costa C, Lopes A, Griz L, Medeiro MA, Bandeira F. Prevalence of osteoporosis and vertebral fractures in postmenopausal women with type 2 diabetes mellitus and their relationship with duration of the disease and chronic complications. J Diabetes Complications. 2011; 25: 216-221.

126. Sato $M$, Ye $W$, Sugihara $T$, Isaka Y. Fracture risk and healthcare resource utilization and costs among osteoporosis patients with type 2 diabetes mellitus and without diabetes mellitus in Japan: Retrospective analysis of a hospital claims database. BMC Musculoskelet Disord. 2016; 17: 489

127. Martinez-Laguna D, Tebe C, Javaid MK, Nogues X, Arden NK, Cooper C, et al. Incident type 2 diabetes and hip fracture risk: A population-based matched cohort study. Osteoporos Int. 2015; 26: 827-833.

128. Schneider ALC, Williams EK, Brancati FL, Blecker S, Coresh J, Selvin E. Diabetes and risk of fracture-related hospitalization: The atherosclerosis risk in communities study. Diabetes Care. 2013; 36: 1153-1158.

129. Napoli N, Strotmeyer ES, Ensrud KE, Sellmeyer DE, Bauer DC, Hoffman AR, et al. Fracture risk in diabetic elderly men: the MrOS study. Diabetologia. 2014; 57: 2057-2065. 
130. Partanen J, Heikkinen J, Jamsa T, Jalovaara P. Characteristics of lifetime factors, bone metabolism, and bone mineral density in patients with hip fracture. J Bone Miner Metab. 2002; 20: 367-375.

131. Starup-Linde J, Lykkeboe S, Gregersen S, Hauge EM, Langdahl BL, Handberg A, et al. Bone structure and predictors of fracture in type 1 and type 2 diabetes. J Clin Endocrinol Metab. 2016; 101: 928-936.

132. Rasmussen NH, Dal J. Falls and fractures in diabetes-more than bone fragility. Curr Osteoporos Rep. 2019; 17: 147-156.

133. Zhao Y, Kachroo S, Kawabata H, Colilla S, Mukherjee J, Fonseca V, et al. Association between hypoglycemia and fall-related fractures and health care utilization in older veterans with type 2 diabetes. Endocr Pract. 2016; 22: 196-204.

134. Kachroo S, Kawabata H, Colilla S, Shi L, Zhao Y, Mukherjee J, et al. Association between hypoglycemia and fall-related events in type 2 diabetes mellitus: Analysis of a U.S. commercial database. J Manag Care Spec Pharm. 2015; 21: 243-253.

135. Puar TH, Khoo JJ, Cho LW, Xu Y, Chen YT, Chuo AM, et al. Association between glycemic control and hip fracture. J Am Geriatr Soc. 2012; 60: 1493-1497.

136. Zhong $\mathrm{N}$, Zhang $\mathrm{Y}, \mathrm{Pu} X, \mathrm{Xu} \mathrm{B}, \mathrm{Xu} \mathrm{M}, \mathrm{Cai} \mathrm{H}$, et al. Microangiopathy is associated with bone loss in female type 2 diabetes mellitus patients. Diab Vasc Dis Res. 2018; 15: 433-441.

137. Holloway-Kew KL, Marijanovic N, De Abreu LLF, Sajjad MA, Pasco JA, Kotowicz MA. Bone mineral density in diabetes and impaired fasting glucose. Osteoporos Int. 2019; 30: 17991806.

138. Barrett-Connor E, Kritz-Silverstein D. Does hyperinsulinemia preserve bone? Diabetes Care. 1996; 19: 1388-1392.

139. Barrett-Connor E, Holbrook TL. Sex differences in osteoporosis in older adults with noninsulin-dependent diabetes mellitus. JAMA. 1992; 268: 3333-3337.

140. Lunt M, Masaryk P, Scheidt-Nave C, Nijs J, Poor G, Pols H, et al. The effects of lifestyle, dietary dairy intake and diabetes on bone density and vertebral deformity prevalence: The EVOS study. Osteoporos Int. 2001; 12: 688-698.

141. Rishaug U, Birkeland KI, Falch JA, Vaaler S. Bone mass in non-insulin-dependent diabetes mellitus. Scand J Clin Lab Invest. 1995; 55: 257-262.

142. Chi JH, Shin MS, Lee BJ. Association of type 2 diabetes with anthropometrics, bone mineral density, and body composition in a large-scale screening study of Korean adults. PLoS One. 2019; 14: e0220077.

143. Patsch JM, Rasul S, Huber FA, Leitner K, Thomas A, Kocijan R, et al. Similarities in trabecular hypertrophy with site-specific differences in cortical morphology between men and women with type 2 diabetes mellitus. PLoS One. 2017; 12: e0174664.

144. Shah VN, Harrall KK, Shah CS, Gallo TL, Joshee P, Snell-Bergeon JK, et al. Bone mineral density at femoral neck and lumbar spine in adults with type 1 diabetes: A meta-analysis and review of the literature. Osteoporos Int. 2017; 28: 2601-2610.

145. Zebaze R, Seeman E. Cortical bone: A challenging geography. J Bone Miner Res. 2015; 30: 2429.

146. Goldin A, Beckman JA, Schmidt AM, Creager MA. Advanced glycation end products: Sparking the development of diabetic vascular injury. Circulation. 2006; 114: 597-605. 
147. Sanguineti R, Puddu A, Mach F, Montecucco F, Viviani GL. Advanced glycation end products play adverse proinflammatory activities in osteoporosis. Mediators Inflamm. 2014; 2014: 975872.

148. Donath MY, Shoelson SE. Type 2 diabetes as an inflammatory disease. Nat Rev Immunol. 2011; 11: 98-107.

149. Calle MC, Fernandez ML. Inflammation and type 2 diabetes. Diabetes Metab. 2012; 38: 183191.

150. Weitzmann MN. The role of inflammatory cytokines, the RANKL/OPG axis, and the immunoskeletal interface in physiological bone turnover and osteoporosis. Scientifica (Cairo). 2013; 2013: 125705.

151. Wheater G, Elshahaly M, Naraghi K, Tuck SP, Datta HK, van Laar JM. Changes in bone density and bone turnover in patients with rheumatoid arthritis treated with rituximab, results from an exploratory, prospective study. PLoS One. 2018; 13: e0201527.

152. Gillespie LD, Gillespie WJ, Robertson MC, Lamb SE, Cumming RG, Rowe BH. Interventions for preventing falls in elderly people. Cochrane Database Syst Rev. 2003: CD000340. doi: 10.1002/14651858.CD000340.

153. Yang $Y, H u X$, Zhang $Q$, Zou R. Diabetes mellitus and risk of falls in older adults: A systematic review and meta-analysis. Age Ageing. 2016; 45: 761-767.

154. Roman de Mettelinge T, Cambier D, Calders P, Van Den Noortgate N, Delbaere K. Understanding the relationship between type 2 diabetes mellitus and falls in older adults: $A$ prospective cohort study. PloS One. 2013; 8: e67055.

155. Morrison S, Colberg SR, Parson HK, Vinik Al. Relation between risk of falling and postural sway complexity in diabetes. Gait Posture. 2012; 35: 662-668.

156. Wu JS, Yang YC, Lu FH, Wu CH, Chang CJ. Population-based study on the prevalence and correlates of orthostatic hypotension/hypertension and orthostatic dizziness. Hypertens Res. 2008; 31: 897-904.

157. van Hateren KJ, Kleefstra N, Blanker MH, Ubink-Veltmaat LJ, Groenier KH, Houweling ST, et al. Orthostatic hypotension, diabetes, and falling in older patients: A cross-sectional study. $\mathrm{Br} \mathrm{J}$ Gen Pract. 2012; 62: e696-e702.

158. Dawson-Hughes B, Bouxsein M, Shea K. Bone material strength in normoglycemic and hyperglycemic black and white older adults. Osteoporos Int. 2019; 30: 2429-2435.

159. Valentini A, Cianfarani MA, De Meo L, Morabito P, Romanello D, Tarantino U, et al. FRAX tool in type 2 diabetic subjects: The use of $\mathrm{HbA1c}$ in estimating fracture risk. Acta Diabetol. 2018; 55: 1043-1050.

160. Zhou L, Song J, Yang S, Meng S, Lv X, Yue J, et al. Bone mass loss is associated with systolic blood pressure in postmenopausal women with type 2 diabetes in Tibet: A retrospective cross-sectional study. Osteoporos Int. 2017; 28: 1693-1698.

161. Hyassat D, Alyan T, Jaddou H, Ajlouni KM. Prevalence and risk factors of osteoporosis among jordanian postmenopausal women attending the national center for diabetes, endocrinology and genetics in jordan. Biores Open Access. 2017; 6: 85-93.

162. Siddapur PR, Patil AB, Borde VS. Comparison of bone mineral density, T-scores and serum zinc between diabetic and non diabetic postmenopausal women with osteoporosis. J Lab Physicians. 2015; 7: 43-48. 
163. Schacter GI, Leslie WD. Spine-hip thickness difference measured by dual-energy X-ray absorptiometry is associated with diabetes mellitus in women and men. J Clin Densitom. 2015; 18: 512-518.

164. Aypak C, Türedi, Bircan MA, Araz M. Association of haematological parameters with bone mineral density in elderly diabetic women. Acta Clin Belg. 2015; 70: 339-344.

165. Karimifar M, Pasha MAP, Salari A, Zamani A, Salesi M, Motaghi P. Evaluation of bone loss in diabetic postmenopausal women. J Res Med Sci. 2012; 17: 1033-1038.

166. Asano M, Fukui M, Hosoda H, Shiraishi E, Harusato I, Kadono M, et al. Bone stiffness in men with type 2 diabetes mellitus. Metabolism. 2008; 57: 1691-1695.

167. Korpelainen R, Korpelainen J, Heikkinen J, Väänänen K, Keinänen-Kiukaanniemi S. Lifelong risk factors for osteoporosis and fractures in elderly women with low body mass index-A population-based study. Bone. 2006; 39: 385-391.

168. Horiuchi T, Kazama H, Araki A, Inoue J, Hosoi T, Onouchi T, et al. Impaired gamma carboxylation of osteocalcin in elderly women with type II diabetes mellitus: Relationship between increase in undercarboxylated osteocalcin levels and low bone mineral density. J Bone Miner Metab. 2004; 22: 236-240.

169. Bauer DC, Browner WS, Cauley JA, Orwoll ES, Scott JC, Black DM, et al. Factors associated with appendicular bone mass in older women. Ann Intern Med. 1993; 118: 657-665.

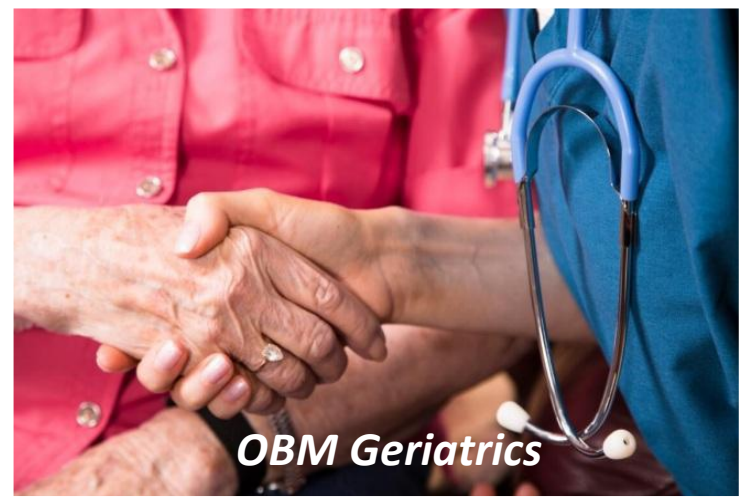

Enjoy OBM Geriatrics by:

1. Submitting a manuscript

2. Joining in volunteer reviewer bank

3. Joining Editorial Board

4. Guest editing a special issue

For more details, please visit:

http://www.lidsen.com/journals/geriatrics 